\title{
STRUCTURAL AND ELECTRONIC PROPERTIES OF GRAPHENE ON 4H- AND 3C-SiC
}

\section{Chamseddine Bouhafs}

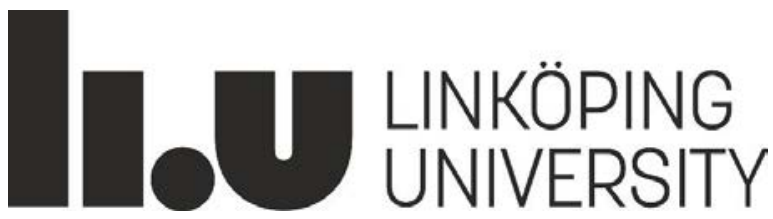

Semiconductor materials Department of Physics, chemistry and Biology

Linköping University Linköping 2016 
(C) Chamseddine Bouhafs, 2016

\section{Cover}

Atomic force microscopy topographic image of graphene on $\mathrm{C}$-face $4 \mathrm{H}-\mathrm{SiC}(000-1)$ grown by high-temperature sublimation in argon atmosphere.

Published paper reprinted with permission from copy right holder American Institute of Physics (Paper I). 
Je dédie cette thèse pour ceux qui sont très loin de moi et très proches à mon coeur:

Pour celle qui vient de l'île des rêves et qui a changè ma vie au plus beau rêve. Pour celle que je dois ma vie.

Pour ma famille.

Pour ceux qui ne perdent jamais la foi en l'humanité. 



\section{ABSTRACT}

Graphene is a one-atom-tick carbon layer arranged in a honeycomb lattice. Graphene was first experimentally demonstrated by Andre Geim and Konstantin Novoselov in 2004 using mechanical exfoliation of highly oriented pyrolytic graphite (exfoliated graphene flakes), for which they received the Nobel Prize in Physics in 2010. Exfoliated graphene flakes show outstanding electronic properties, e.g., very high free charge carrier mobility parameters and ballistic transport at room temperature. This makes graphene a suitable material for next generation radio-frequency and terahertz electronic devices. Such applications require fabrication methods of large-area graphene compatible with electronic industry. Graphene grown by sublimation on silicon carbide $(\mathrm{SiC})$ offers a viable route towards production of large-area, electronic-grade material on semi-insulating substrate without the need of transfer. Despite the intense investigations in the field, uniform wafer-scale graphene with very high-quality that matches the properties of exfoliated graphene has not been achieved yet. The key point is to identify and control how the substrate affects graphene uniformity, thickness, layer stacking, structural and electronic properties. Of particular interest is to understand the effects of $\mathrm{SiC}$ surface polarity and polytype on graphene properties in order to achieve large-area material with tailored properties for electronic applications. The main objectives of this thesis are to address these issues by investigating the structural and electronic properties of epitaxial graphene grown on $4 \mathrm{H}-\mathrm{SiC}$ and $3 \mathrm{C}-\mathrm{SiC}$ substrates with different surface polarities.

The first part of the thesis includes a general description of the properties of graphene, bilayer graphene and graphite. Then, the properties of epitaxial graphene on $\mathrm{SiC}$ by sublimation are detailed. The experimental techniques used to characterize graphene are described. A summary of all papers and contribution to the field is presented at the end of Part I. Part II consists of seven papers.

Paper I reports on the structural, vibrational, and dielectric function properties of graphene grown on the $\mathrm{C}$-face of $4 \mathrm{H}-\mathrm{SiC}(000-1)$ with surface defects. We have shown that the average number of graphene layers, the size of the domains with uniform thickness and the crystallite size increase with the increase of temperature. This improved graphene quality is attributed to an enhanced sublimation of $\mathrm{Si}$ from the $\mathrm{SiC}$ and to the elimination of $\mathrm{SiC}$ surface defects by surface restructuring during the sublimation growth. Central result of the paper is the transition from decoupled to Bernal stacked graphene layers with the increase in growth temperature, which is explained by a competition between growth mechanisms. We also determined the thickness dependence of graphene dielectric function. Fi- 
nally, we reported the first observation of an interface layer that forms between $\mathrm{SiC}$ and graphene and it is composed of amorphous carbon, silicon and oxygen. We have established the evolution of the interface layer with growth temperature and outlined its implications for potential applications of graphene.

Paper II reports a detailed study of the interface layer properties and its evolution with time for a few layer graphene (FLG) sample with large homogeneous areas of monolayer (ML), bi-layer (BL) and tree-layer (TL) on the C-face of $4 \mathrm{H}-\mathrm{SiC}(000-1)$ without any defects. $\mathrm{A} \mathrm{SiO}_{x}$ layer is identified at the interface between graphene and $\mathrm{SiC}$ independently of graphene thickness and domain size. We have found that the chemical composition of the interface layer changes towards $\mathrm{SiO}_{2}$ and its thickness increases with aging in normal ambient conditions. We have shown that the presence of the interface layer causes the formation of nonideal Schottky contact behavior for electrical transport between ML graphene and $\mathrm{SiC}$, which has significant implications for device applications. Finally, BL and FLG are shown to be composed of decoupled graphene layers. However, contrary to previous works on decoupled C-face graphene, our investigations indicate that the adjacent layers in the BL and FLG stacks may not be rotationally disordered.

Paper III reports Landau level spectroscopy, measured using mid-infrared optical Hall effect in combination with low energy electron microscopy and lowenergy electron diffraction investigations of FLG grown on the C-face of 3C-SiC (111). We show for the first time that FLG on 3C-SiC (111) consists of decoupled graphene sheets and behaves effectively as a single layer graphene with linearly dispersing bands (Dirac cones) at the graphene K point. Low-energy electron diffraction mapping suggests that the azimuth rotation occurs between adjacent domains within the same sheet rather than vertically in the stack.While the origin of the decoupling between the individual sheets still remains unknown, the fact that FLG on 3C-SiC(111) behaves as a single layer graphene with carrier mobility of $33000 \mathrm{~cm}^{2} \mathrm{~V}^{-1} \mathrm{~s}^{-1}$ at $1.5 \mathrm{~K}$ gives good prospects for its applications in future electronic devices.

Paper IV reports on the Landau level (LL) splitting in epitaxial graphene grown on the C-terminated face of $4 \mathrm{H}-\mathrm{SiC}(000-1)$. Infrared optical Hall effect is employed to measure LL spectroscopy in reflection mode. We find that the LL transitions in epitaxial graphene exhibit polarization preserving selection rules and the transition energies obey a square-root dependence on the magnetic field strength for the two sets of LL. We find Fermi velocity values of $v_{F 1}=1.03 \times 10^{6} \mathrm{~ms}^{-1}$ and $v_{F 2}=1.09 \times 10^{6} \mathrm{~ms}^{-1}$ for the two sets, respectively. The origin of the LL splitting is discussed and it is suggested that the most likely explanation is the appearance of extra localized levels due to vacancy defects. 
Paper V reports on cavity-enhanced optical Hall effect experiments at terahertz $(\mathrm{THz})$ frequencies to determine the free charge carrier properties in graphene with different number of layers grown on Si-face $4 \mathrm{H}-\mathrm{SiC}(0001)$. We have found that i) monolayer graphene possesses p-type conductivity with a free hole concentration in the low $10^{12} \mathrm{~cm}^{-2}$ range and a free hole mobility parameter as high as $1550 \mathrm{~cm}^{2} \mathrm{~V}^{-1} \mathrm{~s}^{-1}$, ii) 6 monolayers graphene shows n-type doping behavior with a much lower free electron mobility parameter of $470 \mathrm{~cm}^{2} \mathrm{~V}^{-1} \mathrm{~s}^{-1}$ and an order of magnitude higher free electron density in the low $10^{13} \mathrm{~cm}^{-2}$ range. The observed differences in the carrier type of the two samples are suggested to be due to increased hydrophobicity of graphene with increasing number of graphene layers, which significantly diminishes adsorption of p-type dopants from the environment. The reduced mobility parameter in the thick graphene sample was attributed to the higher free charge carrier density, higher effective mass and enhanced scattering between the graphene sheets in the thick graphene. The cavity-enhanced $\mathrm{THz}$ optical Hall effect is demonstrated to be an excellent tool for contactless access to the type of free charge carriers and their properties in two-dimensional materials such as graphene.

Paper VI reports the first in-situ experiments on the effect of ambient gas exposure on the free charge carrier concentration and mobility of monolayer graphene on $4 \mathrm{H}-\mathrm{SiC}(0001)$ using contactless in-situ cavity-enhanced $\mathrm{THz}$ optical Hall effect. The exposure of graphene to cycles of different inert gases (for example: helium and nitrogen) and air, shows that exposure to ambient causes an initial rapid change in the carrier properties, followed by a gradual change for the rest of the exposure time. This indicates that the sample could take longer than one day to reach its final ambient state. Furthermore, we have found that exposure to an inert gas reverses the electron withdrawal caused by air. The doping trend is essentially repeatable when switching between inert gases and air. We found that the mobility variation as a function of sheet density obtained from the different doping cycles shows a linear dependence. This suggests that the dominant scattering mechanism related to ambient doping originats from charged impurities. The charge impurities are presumed to be by products of the doping redox reaction involving $\mathrm{O}_{2}, \mathrm{H}_{2} \mathrm{O}$, and $\mathrm{CO}_{2}$ caused by ambient exposure.

Paper VII reports a new crucible design for the sublimation growth of graphene and the optimization of growth conditions to achieve large-area homogenous monolayer graphene on Si-face $4 \mathrm{H}-\mathrm{SiC}(0001)$. The effect of buffer layer growth temperature on the thickness homogeneity and free charge carrier properties is established. We show that at the best conditions $99 \%$ monolayer graphene with uniform surface morphology can be grown over areas of $15 \times 15 \mathrm{~mm}^{2}$. 
viii

\section{POPULÄRVETENSKAPLIG}

Materialvetenskapen har fått en uppmrksamhet utöver det vanliga ända sedan upptäckten av grafen år 2004. En stor mängd fysikaliska fenomen och potentiella applikationer associerade till grafens unika natur har föranlett en forskningsaktivitet utan motstycke. Grafen är mirakelmaterialet vars upphovsmakare, Novoselov och Geim, blev tilldelade Nobelpriset i fysik år 2010. Denna tvådimensionella kristall bestående av endast ett atomlager kol arrangerat likt en bivaxkaka är stabil i normal omgivning. Grafen har dessutom hög elektronmobilitet, hög elektrisk och termisk ledningsförmåga, optiskt transparent samt hög mekanisk tålighet. Utöver detta är grafen en semi-metall innehavande av en linjär energispridning, även kallat Dirac cone. Detta medför en spännande avvikelse i kontrast till konventionella halvledare och är därför mycket intressant inom framtida halvledarapplikationer. Den verkliga utmaningen är däremot uppskalningen från forskningsnivå till produktionsnivå i industrin. Ett av de stora problemen idag är att de mest enastående egenskaperna har uppmätts på mekaniskt exfolierat grafen vilket är den metod som framställer materialet i högsta kvalitet. Den metoden är inte tillämpbar för storskalig produktion, därför behövs ett alternativ, nämligen epitaxiell tillväxt av grafen med önskvärda egenskaper. För att åstadkomma detta har jag i min avhandling fokuserat på tillväxt och karaktärisering av epitaxiell grafen på SiC via sublimering. Metoden för högtemperatur upplösning av $\mathrm{SiC}$ för att bilda grafen på dess yta har utvecklats av Rositsa Yakimova och kan leverera material med hög homogenitet som är kompatibel med industriell framställning. I avhandlingen presenterar jag en egenutvecklad smältdegel designad för tillväxt av grafen, själva tillväxten av materialet samt karaktäriseringen av de strukturella egenskaperna. Ett antal olika karaktäriseringstekniker har använts i detta syfte för att belysa och optimera grafenets strukturella och elektroniska egenskaper. Detta inkluderar spektroskopisk ellipsometri och optiska Hall-effekten, Raman spektroskopi, röntgen-fotoelektron-spektroskopi / mikroskopi, atomkraftsmikroskopi, kelvinsondmikroskopi, vridningsresonant ledande atomkraft-mikroskopi, transmissionselektronmikroskopi, lågenergielektronmikroskopi, och lågenergi-elektrondiffraktion. 


\section{PREFACE}

This Doctoral thesis is the results of my doctoral studies in Semiconductor materials division at the department of physics, chemistry and biology (IFM) at Linköping University between 2012 and 2016. The main objective of this thesis is to investigate the structural and electronic properties of graphene on $4 \mathrm{H}-\mathrm{SiC}(0001)$ and on 3C-SiC(111) with different surface polarities. The thesis also includes designing a crucible with improved temperature homogeneity and a optimization of growth conditions for a monolayer graphene on the Si-face of $4 \mathrm{H}-\mathrm{SiC}(0001)$ substrates. The results are presented in seven papers proceeded by an introduction. This thesis was financed by: the Marie Curie actions under the Project No.264613NetFISiC, the centre of Nano Science and Nano technology (CeNano), the Swedish Research Council (VR Contract 2013 - 5580), the Swedish Governmental Agency for Innovation Systems (VINNOVA) under the VINNMER international qualification program Grant No.2011 - 03486, the Swedish foundation for strategic research (SSF) under Grants No. FFL12 - 0181 and No. RIF14 - 055, the Swedish Government Strategic Research Area in Materials Science on Functional Materials at Linköping University (Faculty Grant SFO Mat LiU No2009 00971). 


\section{INCLUDED PAPERS}

\section{- Paper I}

Structural properties and dielectric function of graphene grown by hightemperature sublimation on $4 \mathrm{H}-\mathrm{SiC}(000-1)$

C. Bouhafs, V. Darakchieva, I. L. Persson, A. Tiberj, P. O. Á.Persson, M. Paillet, A.-A. Zahab, P. Landois, S. Juillaguet, S. Schöche, M. Schubert, and R. Yakimova.

Journal of Applied Physics 117, 085701 (2015)

\section{- Paper II}

Interface and electrical properties, ordering and decoupling of few layer graphene on C-face $4 \mathrm{H}-\mathrm{SiC}$

C. Bouhafs, A. A. Zakharov, I. G. Ivanov, F. Giannazzo, J. Eriksson, V. Stanishev, P. Kühne, T. Iakimov, T. Hofmann, M. Schubert, F. Roccaforte, R. Yakimova, V. Darakchieva.

Carbon, under review

\section{- Paper III}

Decoupling and ordering of multilayer graphene on C-face 3C-SiC(111)

C. Bouhafs, V. Stanishev, A. A. Zakharov, T. Hofmann, P. Kühne, T. Iakimov, R. Yakimova, M. Schubert, V. Darakchieva.

Applied Physics Letters, under review

\section{- Paper IV}

On the Landau level splitting in few layer epitaxial graphene

C. Bouhafs, P. Kühne, V. Stanishev, I. G. Ivanov, T. Iakimov, R. Yakimova, M. Schubert, T. Hofmann and V. Darakchieva.

Manuscript

\section{- Paper V}

Cavity-enhanced optical Hall in epitaxial graphene detected at terahertz frequencies

N. Armakavicius, C. Bouhafs, V. Stanishev, P. Kühne, R. Yakimova, S. Knight, 
T. Hofmann, M. Schubert, and V. Darakchieva.

Applied Surface Science, in press

(DOI: 10.1016/j.apsusc.2016.10.023)

\section{- Paper VI}

Terahertz optical Hall effect and cyclic gas doping reveal impurity-induced carrier scattering mechanism in epitaxial graphene

S. Knight, C. Bouhafs, T. Hofmann, N. Armakavicius, P. Kühne, V. Stanishev,

I. G. Ivanov, R. Yakimova, S. Wimer, V. Darakchieva and M. Schubert.

Manuscript

- Paper VII

Effect of buffer layer growth conditions on the properties of monolayer graphene on Si-face $4 \mathrm{H}-\mathrm{SiC}$

V. Stanishev, C. Bouhafs, A. A. Zakharov, I. G. Ivanov, N. Armakavicius, P. Kühne, R. Yakimova, and V. Darakchieva.

Manuscript

\section{MY CONTRIBUTION TO THE PAPERS}

\section{- Paper I}

I contributed to the planning. I have participated in the measurements of micro-Raman, the AFM measurements, in the discussion of the TEM results. I have analyzed the spectroscopic data ellipsometry with my supervisor and the Raman data and i have written the first version of the paper.

\section{- Paper II}

I have participated in the measurements of conductive-AFM, Kelvin probe, Raman, reflectance, micro- LEED and LEEM. I have analyzed micro- LEED, LEEM, XPS, Raman, reflectance, Kelvin probe and mid-infrared optical Hall effect data. I have interpret the measurements together with my co-authors. I have written the first version of the paper. 


\section{- Paper III}

I have analyzed the micro- LEED, LEEM and mid-infrared optical Hall effect data. I have written the first version of the paper.

\section{- Paper IV}

I have performed the Raman polarization measurements. I have interpreted the data together with my supervisor.

\section{- Paper V}

I have participated in the growth of the graphene samples and reflectance measurements. I took part in the discussion of the results.

\section{- Paper VI}

I have participated in the growth of the graphene samples, the reflectance and Raman measurements. I have participated to the building of the experimental setup. I took part in the interpretation and the discussion of the results.

\section{- Paper VII}

I have participated the optimization of the growth. I have participated in the growth of the graphene samples and reflectance, AFM and Raman measurements. I have participated in the reflectance and Raman data analysis. I took part in the interpretation and the discussion of the results.

\section{RELATED BUT NOT INCLUDED PAPERS}

- Morphological and electronic properties of epitaxial graphene on SiC

R. Yakimova, T. Iakimov, G. R. Yazdi, C. Bouhafs, J. Eriksson, A. Zakharov, A. Boosalis, M. Schubert, and V. Darakchieva

Physics B, 439, 59 (2014) 


\section{NOT RELATED AND NOT INCLUDED PAPERS}

- Effect of nitrogen on the $\mathrm{GaAs}_{0.9-x} \mathrm{~N}_{x} \mathrm{Sb}_{0.1}$ dielectric function from the near-infrared to the ultraviolet

N. Ben. Sedrine, C. Bouhafs, J.C. Harmand, R. Chtourou, V. Darakchieva. Applied Physics Letters 97, 201903 (2010).

- Optical properties of $\mathrm{GaAs}_{0.9-x} \mathrm{~N}_{x} \mathrm{Sb}_{0.1}$ alloy films studied by spectroscopic ellipsometry

N. Ben. Sedrine, C. Bouhafs, M. Schubert, J.C. Harmand, R. Chtourou, V. Darakchieva.

Thin Solid Films 519 (9), 2838-2842 (2011). 


\section{ACKNOWLEDGMENTS}

During the last 5 years, I have learned a lot as a PhD student at the Semiconductor Materials Division at IFM (Linköping University), both at the scientific and personal levels. I am truly grateful for the knowledge and the experience I acquired during this time, thanks to the help and support of few persons that I would like to sincerely acknowledge.

First of all, I would like to thank my supervisor Prof. Vanya Darakchieva for giving me the opportunity to do my PhD at Linköping University under her supervision, for her patience, invaluable guidance and encouragements, and for financial support.

I would like to thank my co-supervisor, Prof. Rozitsa Yakimova, for providing the sublimation growth facilities, for sharing her invaluable knowledge and for financial support.

I would like also to thank my co-supervisor Ivan G. Ivanov for giving me the opportunity to perform my research in the optical characterization laboratory, for his guidance and help.

I am deeply thankful to Philipp Kühne, for his help, encouragements and discussions in the optical Hall effect experiments, data modeling and constructive criticism.

I would like to express a special thank to Alex A. Zakharov for the LEEM, LEED and XPS experiments at Lund University and for the fruitful discussions.

I would like to thank Vallery Stanishev for his help and professionalism.

I would like to thank Filippo Giannazzo and Fabrizio Roccaforte for inviting me to perform the electrical measurements at CNR-Institute for Microelectronics and Microsystems Catania (Catania, Italy).

Prof. Per Person and Ingemar Persson are acknowledged for their collaboration with Transmission electron microscopy.

Jens Eriksson is greatly acknowledged for the help and discussions about the Kelvin probe measurements.

I would like thank Prof. Mathias Schubert and Tino Hofmann for fruitful discussions.

I also thank Sandrine Juillaguet and Antoine Tiberj at Montpellier (France) for inviting me the opportunity to perform Raman measurements.

I would like to direct a special thank to the head of Division Prof. Erik Janzén and to the director of studies Prof. Per Olof Holtz for giving me the conditions to pursue my research plan and my courses.

All the administrative and technical staff at IFM are acknowledged as well, and a 
special thanks goes to Eva Wibom and Louise Gustafsson.

I would like express my gratitude to all present and former members of the Semiconductor Materials Division at IFM, especially to my colleagues Valdas Jokubavicius, Volodymyr Khranovskyy, Ian Booker, Gholam Reza Yazdi, Tihomir Iakimov, Prof. Nguyen Tien Son, Jawad ul Hassan, Prof. Leif Johansson, Prof. Anne Henry, Camille Pallier. You have all contributed to a creative and pleasant working environment and always been there if help was needed.

I would like to thank my friends at IFM: Houssaine Machhadani, Nerijus Armakavicius, Kevin Mead, Martin Eriksson, Daniel Nilsson, Chao Xia, Mohammed Ahmad, Ana-Beatriz Chaar, Alexandre Khaldi, Ali Maziz and Arnaud lefebvrier.

Finally, I would like to thank my colleagues and friends of the NetFISiC Marie Curie Network for the nice network environment, their friendship and for the unforgettable memories. 



\section{Contents}

1 Introduction to graphene $\quad$ I

1.1 Crystal and electronic structure of graphene . . . . . . . . . . I

1.2 Bilayer graphene . . . . . . . . . . . . . . . 4

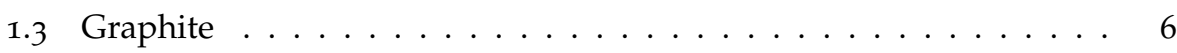

1.4 Vibrational properties of graphene . . . . . . . . . . . . 7

1.5 Optical properties of graphene . . . . . . . . . . . . . 7

1.6 Transport properties of graphene . . . . . . . . . . . . 10

1.7 Graphene and graphite in magnetic field . . . . . . . . . . . . 11

2 Epitaxial graphene on $\mathrm{SiC} \quad 15$

2.1 Introduction . . . . . . . . . . . . . . 15

2.2 Background of epitaxial graphene on $\mathrm{SiC} \ldots \ldots \ldots . . \ldots 18$

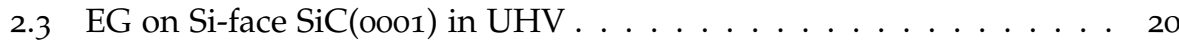

2.4 Graphene on C-face SiC(ooo-1) in UHV . . . . . . . . . . . . . . 21

2.5 Low-temperature EG on Si-face SiC (ooor) . . . . . . . . . . . . . 23

2.6 Low-temperature graphene on $\mathrm{C}$-face $\mathrm{SiC}($ ooo- 1 ) . . . . . . . . . . 24

2.7 High-temperature EG on Si-face SiC (оoо1) . . . . . . . . . . . . . . . 29

2.8 High-temperature graphene on $\mathrm{C}$-face $\mathrm{SiC}($ ooo-1) . . . . . . . . . 31

2.9 Si- and C-face graphene on $3 \mathrm{C}-\mathrm{SiC}(111) \ldots \ldots . \ldots . \ldots 32$

2.10 Samples studied in the thesis . . . . . . . . . . . . . 34

3 Experimental techniques $\quad 35$

3.1 Raman scattering Spectroscopy . . . . . . . . . . . . . . . . . . . . . . 35

3.1.1 The effects of doping ................ 36

3.1 .2 The effects of strain . . . . . . . . . . . 37

3.1.3 The effects of number of layers and stacking . . . . . . . 38

3.1.4 Raman Spectroscopy of graphene on $\mathrm{SiC} \ldots \ldots . . . . . . .38$

3.2 Reflectance . . . . . . . . . . . . . . . . . . . 39 
3.3 Scanning probe microscopy techniques . . . . . . . . . . 40

3.3.1 Atomic force microscopy . . . . . . . . . . . . 40

3.3.2 Conductive atomic force microscopy . . . . . . . . . . . 41

$3 \cdot 3.3$ Scanning Kelvin probe microscopy . . . . . . . . . . . . . . . . 42

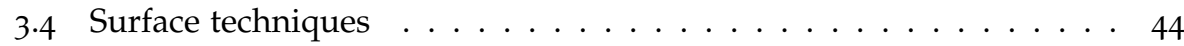

3.4.1 Low-energy electron microscopy . . . . . . . . . . . . 44

3.4 .2 Low-energy electron diffraction . . . . . . . . . . . . 46

3.4.3 X-ray photoelectron spectroscopy and microscopy . . . . . . 47

3.5 Spectroscopic ellipsometry . . . . . . . . . . . . . . . 48

3.5.1 Types of ellipsometry . . . . . . . . . . . . 48

3.5.1.1 Standard spectroscopic ellipsometry . . . . . . . . 48

3.5.1.2 Generalized spectroscopic ellipsometry . . . . . . . 48

3.5 .2 Optical Hall effect . . . . . . . . . . . . . . 50

3.5.2.1 The Optical Hall effect . . . . . . . . . . . . 50

3.5.2.2 Cavity-enhanced and in-situ cavity-enhanced $\mathrm{THz}$ optical Hall effect . . . . . . . . . . . . . . . . . . . . . . . . . . . . . . .

3.5 .3 Optical models . . . . . . . . . . . . . . . 52

3.5.3.1 Dielectric tensor ............... 53

3.5.3.2 Visible to vacuum ultraviolet optical models . . . . 53

3.5.3.3 Mid-infrared/terahertz optical models . . . . . . . 54

3.5 .4 Instrumentation . . . . . . . . . . . . 57

4 Summary of the results $\quad 59$

$\begin{array}{ll}\text { References } & 61\end{array}$

$\begin{array}{ll}\text { List of Figures } & 73\end{array}$ 


\section{Chapter 1}

\section{Introduction to graphene}

A. Geim and K. Novoselov reported the isolation of graphene using mechanical exfoliation in 2004. They first experimentally demonstrated the outstanding physical properties of graphene, such as massless Dirac fermions with extremely high mobility parameters. In 2010, A. Geim and K. Novoselov were awarded the Nobel prize for Physics for groundbreaking experiments regarding the two-dimensional material graphene [1].

\subsection{Crystal and electronic structure of graphene}

The outstanding properties of graphene originate from its unique two-dimensional lattice structure. Graphene consists of a single layer of $s p^{2}$-hybridized carbon atoms that are arranged in a honeycomb lattice. The crystal lattice of graphene contains two triangular sub-lattices composed of atoms A and B, as shown in Fig.1.1(a). The unit cell of graphene (black-dotted line) is rhombic and contains two non equivalent carbon atoms per unit cell (A and B) with unit cell vectors

$$
\overrightarrow{a_{1}}=\left(\frac{3}{2} a-\frac{\sqrt{3}}{2} a\right) \quad \text { and } \quad \overrightarrow{a_{2}}=\left(\frac{3}{2} a, \frac{\sqrt{3}}{2} a\right)
$$

where $a=1.42 \AA$ is the carbon-carbon bond length. The reciprocal lattice of graphene is also hexagonal as shown in Fig.1.1(b). The corresponding reciprocal lattice vectors are

$$
\overrightarrow{g_{1}}=\frac{4 \pi}{3 \sqrt{3} a}\left(\frac{\sqrt{3}}{2},-\frac{3}{2}\right) \quad \text { and } \quad \overrightarrow{g_{2}}=\frac{4 \pi}{3 \sqrt{3} a}\left(\frac{\sqrt{3}}{2}, \frac{3}{2}\right)
$$

The high symmetry points in the first Brillouin zone (BZ) (gray hexagon in Fig. 1.1(b)) are the $\Gamma$ point at the zone center (origin of the reciprocal space with 

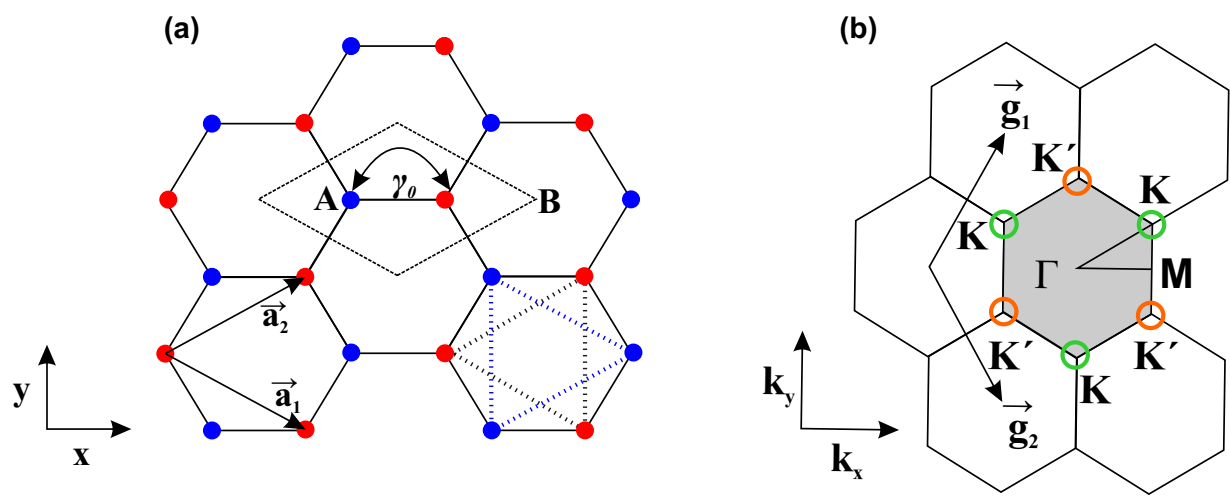

Figure 1.1: (a) Periodic lattice of graphene. The unit cell is indicated by dashed lines and the two sub-lattices composed of atoms A and B are shown by dotted triangles. $\gamma_{0}$ is the hopping energy between the nearest neighbour carbon atoms. (b) Reciprocal lattice and high symmetry points of graphene. The first BZ is indicated in gray.

$k=0$ ), the $\mathbf{M}$ point in the middle of the hexagonal sides, and the two non equivalent $\mathbf{K}=\left(\frac{2 \pi}{3 a}, \frac{2 \pi}{3 \sqrt{3} a}\right)$ and $\mathbf{K}^{\prime}=\left(\frac{2 \pi}{3 a},-\frac{2 \pi}{3 \sqrt{3} a}\right)$ points at the hexagon corners. The $\mathbf{K}$ and $\mathbf{K}^{\prime}$ points are known as the Dirac points.

An isolated carbon atom has the configuration of $1 s^{2} 2 s^{2} 2 p^{2}$ and it possesses four valence electrons to form chemical bonds. In graphene, three out of the four valence electrons are hybridized into three $\mathrm{sp}^{2}$ orbitals and form in-plane $\sigma$-bonds, while the remaining electron of each carbon atom forms $\pi$ - (or $\mathrm{P}_{z^{-}}$) orbitals perpendicular to the graphene plane. The $\sigma$-bond is the strongest covalent chemical bond, which is the reason for graphene outstanding mechanical properties [2]. The $\pi$-orbitals determine the low-energy electronic structure of graphene and are responsible for its high electrical conductivity.

The electronic structure of graphene can be calculated using a tight binding model as demonstrated first by P. R. Wallace [3]. The tight binding model only takes into account the interaction between the nearest neighbor carbon atoms (A-B) with hopping energy $\gamma_{0}$. The energy dispersion relation obtained from tight binding model calculations gives

$$
E(\vec{k})= \pm \gamma_{0} \sqrt{1+4 \cos ^{2}\left(\frac{\sqrt{3} k_{y} a}{2}\right)+4 \cos \left(\frac{3 k_{x} a}{2}\right) \cos \left(\frac{\sqrt{3} k_{y} a}{2}\right)}
$$

where $\gamma_{0}=3.12-3.15 \mathrm{eV}$ is the nearest neighbor hopping energy [3], $k_{x}$ and $k_{y}$ are the $\vec{k}$ momentum components in the $x$ and $y$ in-plane directions. Fig.1.2 shows the 


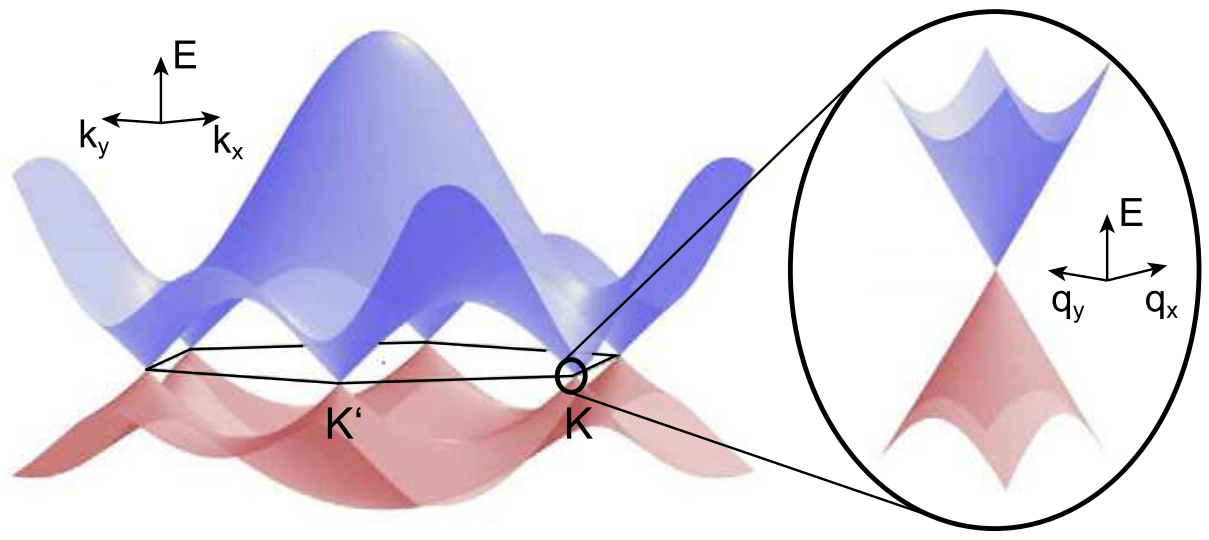

Figure 1.2: Electronic structure of graphene. The valence band (lower red band) and conduction band (upper blue band) touch at the K-points of the BZ. In the vicinity of these points, the energy dispersion relation is linear. Reprinted with permission from Ref. 4 .

electronic structure of graphene obtained from Eqn. 1.3, where the conduction and valence bands are connected at the six non equivalent $\mathbf{K}$ and $\mathbf{K}^{\prime}$ points of the first BZ. Therefore, graphene is considered as a gapless semiconductor or a semimetal. For undoped graphene, the Fermi energy lies exactly at the Dirac points. Close to the $\mathbf{K}$ and $\mathbf{K}^{\prime}$ points, where $\vec{k}=\overrightarrow{\mathbf{K}}+\delta k$, the graphene band structure can be described by

$$
E_{ \pm}(\vec{k})= \pm \hbar v_{f}|\overrightarrow{\delta k}|
$$

where $|\overrightarrow{\delta k}| \ll|\vec{K}|$ and $v_{f}$ is the Fermi velocity, given by

$$
v_{f}=\frac{3 \gamma_{0} a}{2 \hbar} \approx 1 \times 10^{6} \mathrm{~ms}^{-1}
$$

Equation 1.4 shows that the electronic structure of graphene exhibits a linear energy dispersion as a function of the wave vector close to the Dirac points. As a result, the free charge carriers in graphene behave exactly as massless relativistic Dirac-fermions (quasi-particles) with speed $v_{f}$. Consequently, the electrons (holes) in graphene near the $\mathbf{K}\left(\mathbf{K}^{\prime}\right)$ points can be described by the Dirac equation instead of the Schrödinger equation, which is used to describe electron (holes) in conventional semiconductors.

$$
H_{\mathbf{K}}= \pm \hbar v_{f} \vec{\sigma} \vec{k}
$$

where $\vec{\sigma}=\left(\sigma_{x}, \sigma_{y}\right)$ are the Pauli matrix vectors. 
(a)

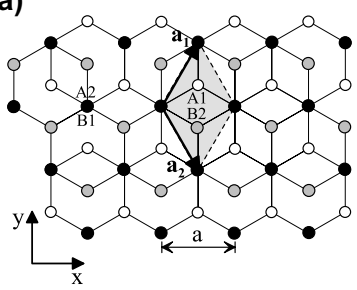

(b)

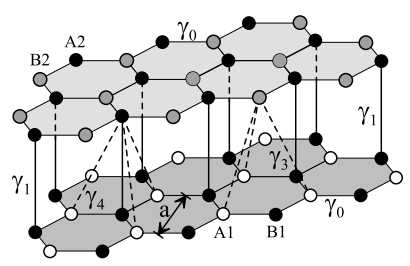

(c)

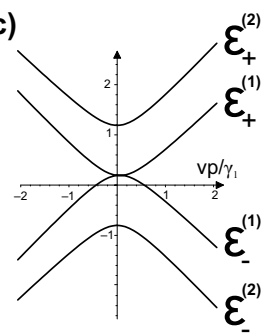

Figure 1.3: (a) Top and (b) side view of the crystal structure of AB-stacked bilayer graphene. Reprinted with permission from Ref. 7. (c) Electronic band structure of bilayer graphene near the $\mathbf{K}$ point obtained by taking into account inter-layer hopping $\gamma_{1}$ and $\gamma_{3}$, zero layer asymmetry and with $v_{3} / v_{f}=0.1$. Reprinted with permission from Ref. 8.

The Dirac fermions in graphene exhibit two-component wave function that describes the sublattices $\mathrm{A}$ and $\mathrm{B}$ with a pseudospin $\pm \sigma$, leading to a different chirality in the graphene conduction and valence bands at the $\mathbf{K}$ and $\mathbf{K}^{\prime}$ points. Therefore, the band structure of graphene at the different Dirac points is not equivalent.

The linear energy dispersion of graphene at the Dirac points is of particular interest since many novel physical phenomena and extraordinary electronic properties found in graphene have been correlated to this unusual band structure. The density of states (DOS) of graphene near the Dirac points is given by [5]

$$
\rho(E)=\frac{g_{s} g_{v}|E|}{2 \pi \hbar^{2} v_{f}^{2}},
$$

where $v_{f}, g_{s}=2$ and $g_{v}=2$ are the Fermi velocity, the spin and the valley degeneracy, respectively. The density of state of graphene increases linearly by increasing $|E|$ and vanishes at $|E|=0$ allowing the possibility of carrier modulation unlike conventional 2D semiconductors with a parabolic electronic structure and a constant DOS $\left(\rho(E)=m^{*} / \pi \hbar^{2}\right.$, where $m^{*}$ is the effective mass). Consequently, graphene can find applications in ambipolar field effect transistors [6].

\subsection{Bilayer graphene}

Bilayer graphene consists of two coupled monolayers that are stacked in a structured order with a layer spacing of $d \approx 0.334 \mathrm{~nm}$. The $A B$ stacking order of bilayer graphene (or Bernal stacking) is the most common and stable phase among all the different reported stacking orders (such as $A A A, A B C$, etc) [9]. In $A B$-stacking 
configuration, the unit cell has four carbon atoms where $\left(A_{1}, B_{1}\right)$ and $\left(A_{2}, B_{2}\right)$ are the non equivalent carbon atoms of the upper and lower layer, respectively (Fig. 1.3(a)). The $A B$-staking is formed when $A_{2}$-type carbon atoms in the upper layer sit on top of $B_{1}$-type carbon atoms of the adjacent lower layer, while the $B_{2}$-type (upper layer) atoms sit over the center of a hexagon, as illustrated in Fig. 1.3(a). The band structure of bilayer graphene can be calculated similarly to the one of graphene using tight binding model $[7,10-12]$. However, in addition to the in-plane interaction between the nearest neighbour carbon atoms of the upper $\left(A_{2}-B_{2}\right)$ and the lower layer $\left(A_{1}-B_{1}\right)$, with hopping energy $\gamma_{A_{1} B_{1}}=\gamma_{A_{2} B_{2}}=\gamma_{0}$ and velocity $v_{f}=3 \gamma_{0} a / 2 \hbar$, the strongest inter-layer interactions $\gamma_{1}, \gamma_{3}$ and $\gamma_{4}$, are included in the model (see Fig. 1.3(b)) [8]. The $A_{1}-B_{2}$ interaction is weak $\left(v_{3} \ll v_{f}\right)$, thus it can be neglected. When $\gamma_{3}=0$, the resulting electronic band structure near the $\mathbf{K}\left(\mathbf{K}^{\prime}\right)$ points obtained from tight binding calculation is given by [8]

$$
\varepsilon_{ \pm}^{(\alpha)}(p)= \pm \sqrt{\frac{\gamma_{1}^{2}}{2}+\frac{\Delta^{2}}{4}+v_{f}^{2} p^{2}+(-1)^{\alpha} \sqrt{\frac{\gamma_{1}^{4}}{4}+\left(\gamma_{1}^{2}+\Delta^{2}\right) v_{f}^{2} p^{2}}}
$$

where $\Delta$ is the asymmetry between on-site energies in the two layers, $\mathrm{p}$ is the momentum close to the $\mathbf{K}\left(\mathbf{K}^{\prime}\right)$ points and $\alpha=1,2$.

$\varepsilon_{ \pm}^{2}(p)$ represent the higher energy conduction and valence bands of bilayer graphene. These bands are the result of the strong inter-layer coupling and are separated by $\gamma_{1}=0.39$ [13]. $\varepsilon_{ \pm}^{1}(p)$ represent the lower conduction and valence bands of bilayer graphene for zero asymmetry $(\Delta=0)$ (Fig. 1.3(c)), which can be approximated by [14]

$$
\varepsilon_{ \pm}^{(1)}(p)= \pm \frac{\gamma_{1}}{2}\left[\sqrt{1+4 v^{2} p^{2} / \gamma_{1}^{2}}-1\right] .
$$

In contrast to monolayer graphene, bilayer graphene shows a parabolic band structure that is connected at $\mathbf{K}\left(\mathbf{K}^{\prime}\right)$ points with a zero bandgap. Consequently, the bilayer graphene can be described as massive Dirac-fermions with an effective mass $m^{*}=\gamma_{1} / 2 v_{f}^{2}$ for which the DOS is independent on the energy.

Interaction between graphene sheets plays an important role in the electronic band structure. Fig. 1.4 shows the electronic band structure obtained by density functional theory of monolayer graphene (dotted line), $A B$-stacked bilayer graphene (dashed line) and twisted bilayer graphene by an angle $\pm 2.204^{\circ}$ (solid line) at the $\mathbf{K}$ point [15]. In contrast to $A B$-stacked bilayer graphene, which has a parabolic band structure, twisted bilayer graphene exhibits a linear band structure similar to monolayer graphene. 


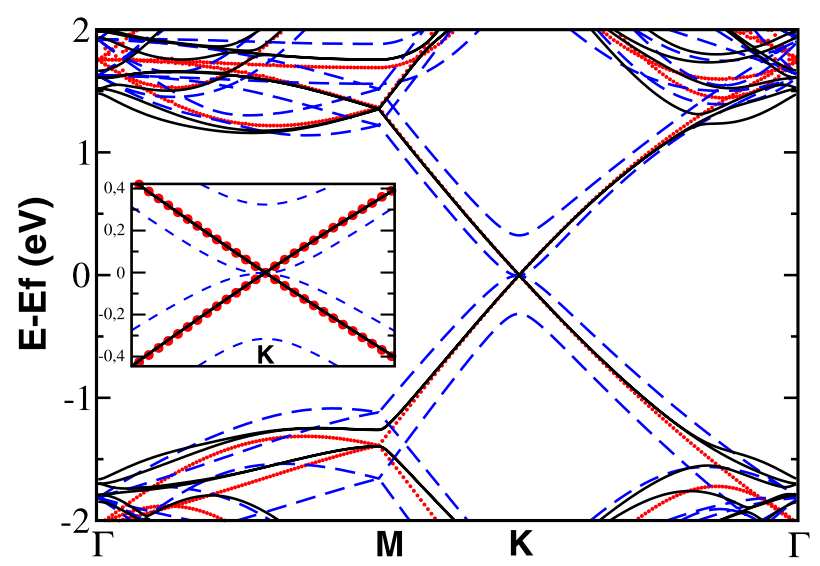

Figure 1.4: Band structure of monolayer graphene (dotted line), $A B$-stacked bilayer graphene (dashed line) and bilayer graphene twisted by an angle $\pm 2.204^{\circ}$ (solid line) calculated by density functional theory. Reprinted with permission from Ref. 15 .

\subsection{Graphite}

Three-dimensional (3D) graphite crystal is composed of $N$ graphene layers $(N \geqslant 10)$ stacked in AB-stacking order as shown in Fig. 1.5(a). The band structure of graphite was investigated by Slonczewski, Weiss and McClure using tight binding calculations (SWM model) [3, 16-18] (see Fig. 1.5(b)). The SWM model for graphite considers two additional interactions between the second-neighbour layers, $\gamma_{2}$ and $\gamma_{5}$ compared to to the tight bonding model for bilayer graphene, as shown in Fig. 1.5(a).

Following the same approach as for bilayer, and neglecting all inter-layer interactions $\left(\gamma_{1}-\gamma_{5}\right)$ except $\gamma_{1}$, the band structure of graphite can be described by [19]

$$
\varepsilon_{ \pm}^{(\alpha)}(p)= \pm \sqrt{\frac{\left(\lambda \gamma_{1}\right)^{2}}{2}+v_{f}^{2} p^{2}+(-1)^{\alpha} \sqrt{\frac{\left(\lambda \gamma_{1}\right)^{4}}{4}+v_{f}^{2} p^{2}\left(\lambda \gamma_{1}\right)^{2}}}
$$

where $\lambda=2 \cos \left(\pi K_{z}\right)$ is the effective coupling.

At the $\mathrm{K}$ point $\left(k_{z}=0, \lambda=2\right)$, graphite shows a parabolic band structure. The electrons in graphite behave similarly as in bilayer graphene (massive-Dirac fermions) with an effective mass $\left(m^{*}=\lambda \gamma_{1} /\left(2 v_{f}^{2}\right)\right)$, two times higher compared to the one of bilayer graphene [19]. At the $\mathbf{H}$ point $\left(k_{z}=0.5, \lambda=0\right)$, the inter-layer coupling between the different graphene layers disappears and graphite shows a linear band structure similarly to graphene. 
(a)

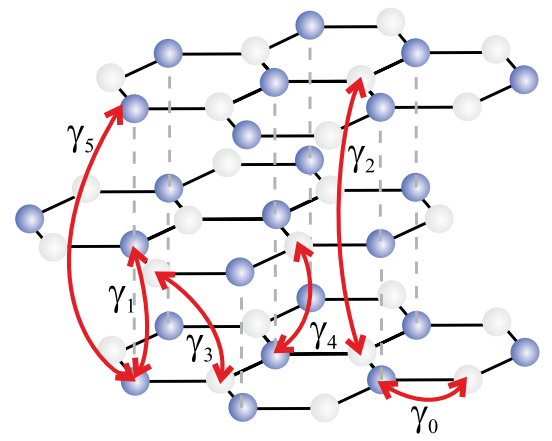

(b)

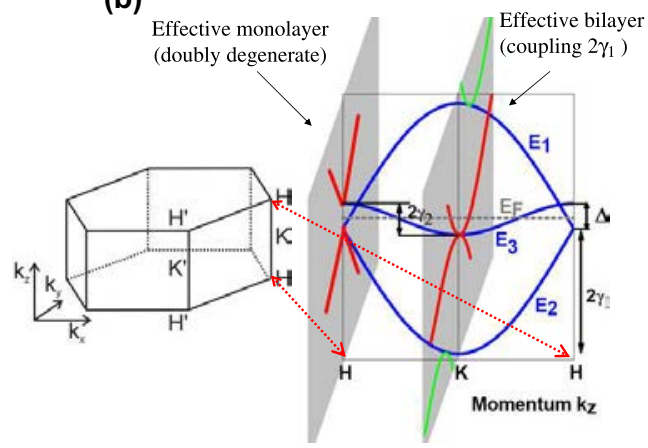

Figure 1.5: (a) Crystal structure of AB-stacked graphite. Different inter-layer interactions $\gamma_{1}-\gamma_{5}$ used in the standard SWM model are indicated. (b) First BZ and electronic band structure of graphite along $\mathbf{H}-\mathbf{K}-\mathbf{H}$ line of the 3D first BZ. Reprinted with permission from Ref. 19.

\subsection{Vibrational properties of graphene}

Monolayer graphene with two atoms per unit cell has six normal vibrational modes from which three are acoustic and three are optical vibrational modes. Fig. 1.6(a) shows the calculated phonon dispersion of graphene. The ZA and ZO modes (branches) correspond the out-of-plane acoustic and optical vibrational mode. The LO and TO modes represent the in-plane longitudinal and transverse optical vibrational mode, respectively. The LO and TO modes describe the vibrations of the graphene sub-lattice A towards the sub-lattice B (See inset in Fig. 1.6(a)) and are degenerated at the $\Gamma$ point [20]. The LA and TA modes represent the in-plane longitudinal and transverse acoustic vibrational mode, respectively. Graphene belongs to the space group P6/ $\mathrm{mmm}$ which corresponds to $\mathrm{D}_{6 h}$ point group. The irreducible representation for the lattice vibrations $\left(\Gamma_{\text {lat.vib }}\right)$ at the $\Gamma$ point is

$$
\Gamma_{\text {lat. vib. }}=A_{2 u}+B_{2 g}+E_{1 u}+E_{2 g}
$$

where $A_{2} u$ and $E_{1 u}$ are acoustic vibration modes, $B_{2 g}$ is out-of-plane optical vibrational mode (silent mode). The $\mathrm{E}_{2 g}$ is the only active Raman mode and correspond to the doubly degenerate $\mathrm{TO}$ and LO phonon mode at the $\Gamma$ point (Fig. 1.6).

\subsection{Optical properties of graphene}

The optical response of graphene depends on the spectral range and doping properties. The optical conductivity of graphene results from both inter-band and 

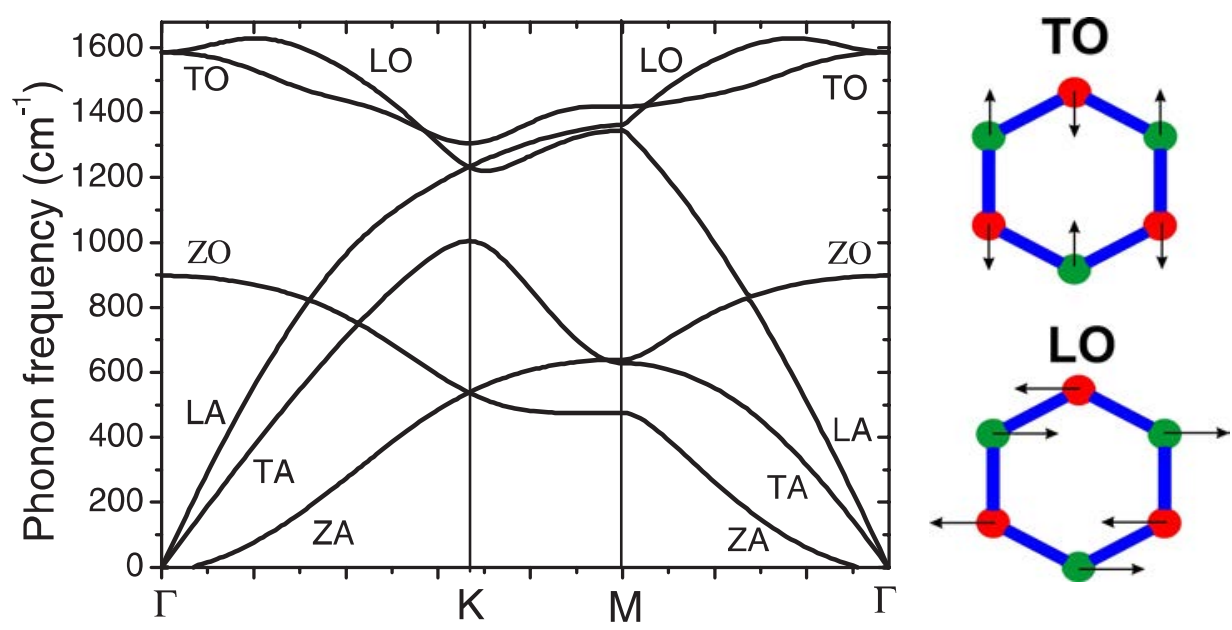

Figure 1.6: Phonon dispersions for monolayer graphene showing the LO, TO, ZO, LA and TA phonon branches. Adapted from Ref. 21. The inset shows $\mathrm{E}_{2 g}$ vibrational modes in graphene.

intra-band processes (free charge carrier absorption). The inter-band transition originates from the transition of an electron from an occupied state in the valence band to an unoccupied state in the conduction band, as shown in Fig. 1.7(a), only when the energy of the incident photon is two-times higher than the Fermi energy of graphene $\left(\hbar \omega>2 E_{f}\right)$. If $\hbar \omega<2 E_{f}$, the inter-band transition does not occur due to Pauli blocking, and in this case the optical conductivity is dominated by intra-band processes (free charge carrier absorption), as illustrated in Figs. 1.7(a) and (b).

For undoped graphene at zero temperature, the calculated optical conductivity $\sigma(\omega)$ near the Dirac points was found to be independent on the frequency of the light $(\omega)$ (i. e. universal optical conductivity) and can be described by [26-28]

$$
\sigma(\omega)=\frac{\pi e^{2}}{2 \hbar}
$$

The absorbance of graphene A $(\omega)$ can be related to the optical conductivity by

$$
A(\omega)=\frac{4 \pi}{c} \sigma(\omega)
$$

As a result of the universal conductivity of graphene, the absorbance of graphene is independent on the frequency $A(\omega)=\pi \alpha \approx 2.29 \%$, where $\alpha$ denotes the fine structure constant. The optical transmittance $T(\omega)$ of graphene is defined by

$$
T(\omega)=1-A(\omega) \approx 1-\pi \alpha
$$


(a)

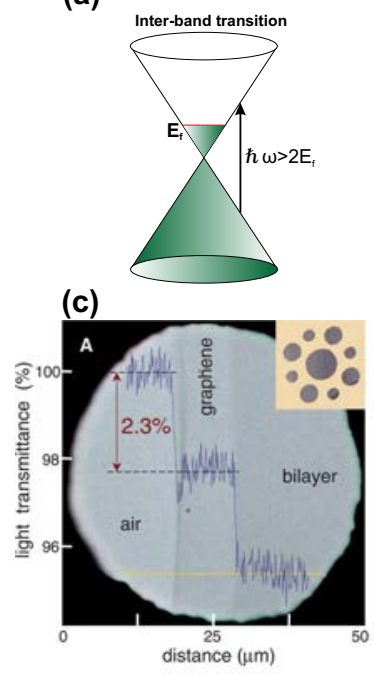

(b)

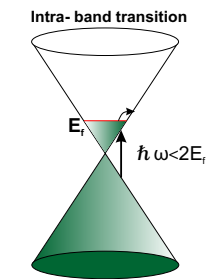

(d)

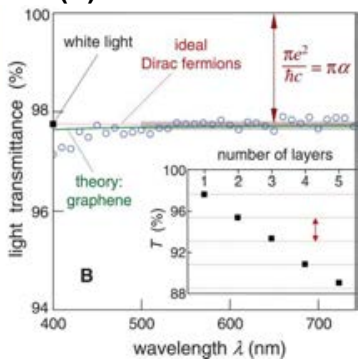

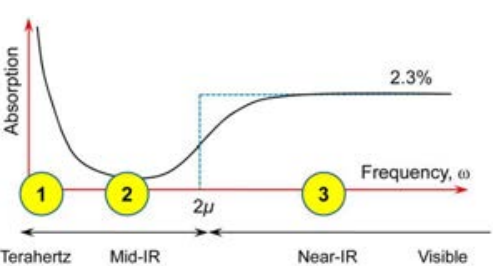

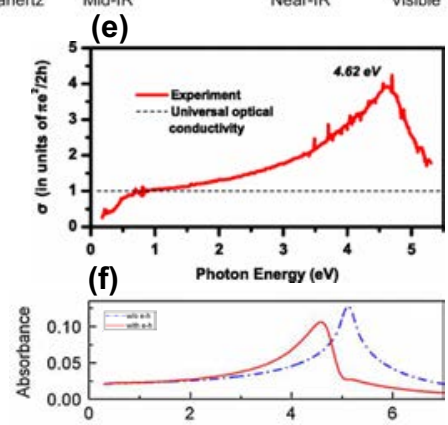

Figure 1.7: (a) Illustration of inter-band and intra-band (free charge carrier absorption) processes. (b) Illustration of a typical absorption spectrum of doped graphene, which shows a universal absorbance of $2.3 \%$ in the visible range, graphene becomes transparent in the mid-infrared region, and shows a strong Drude absorption peak in the far-infrared and $\mathrm{THz}$ frequency regions ( $\mu$ refers here to the Fermi energy). Reprint with permission from Ref. 22. (c) Photograph of a $50 \mu \mathrm{m}$ aperture partially covered with monolayer and bilayer graphene. The line scan profile shows the intensity of transmitted white light along the yellow line. (d) Transmittance spectrum of monolayer graphene (open circles) and comparison with the theoretical transmission (Eqn. 1.14) (red line). Reprint with permission from Ref. 23. (e) Measured optical absorbance of monolayer graphene in the spectral range of 0.2 to $5.5 \mathrm{eV}$. The experimental spectrum shows an absorption peak energy at $4.62 \mathrm{eV}$. Reprint with permission from Ref. 24. (f) GW ab-initio calculations of the optical absorbance of graphene with (solid-blue line) and without (red-dashed line) excitonic effects. Reprint with permission from Ref. 25.

The universal optical conductivity (i.e. absorbance) of graphene was experimentally confirmed for suspended graphene $[23,29]$ in the spectral range of $0.5-1.0 \mathrm{eV}$ (Fig. 1.7(d)). Figs. 1.7 (c) and (d) show the transmittance of suspended exfoliated monolayer and bilayer graphene membranes, in which the absorbance of a monolayer is $2.3 \pm 0.1 \%$. Therefore, the absorbance increases with the graphene thickness by $2.3 \%$ for each graphene layer.

In the ultraviolet region, the optical conductivity (i.e. absorbance) becomes dependent on the frequency and shows a large asymmetric peak absorption at $4.62 \mathrm{eV}$ (see Fig. 1.7(f)). The GW ab-initio calculations demonstrated that this absorption peak originates from the inter-band transitions near the saddle point 
singularity at the M-point (Van Hove singularity) and that the absorption peak is symmetric and occurs at $5.1 \mathrm{eV}$ [25]. The redshift of the peak near $4.5 \mathrm{eV}$ and the different line shape was attributed to the strong resonant excitonic effects (see Fig. 1.7(e)).

In this thesis, we have investigated the optical properties of graphene on $4 \mathrm{H}-\mathrm{SiC}(000 \overline{1})$ grown by sublimation technique using spectroscopic ellipsometry in the visible to vacuum ultraviolet spectral range. We have found that graphene exhibits a critical point transition energy $(\mathrm{CP})$ at $4.51 \mathrm{eV}$, associated with exciton enhanced van Hove singularity. We have investigated the graphene thickness dependence of the graphene dielectric function and we have found that the the $\mathrm{CP}$ point transition energy redshifts with increasing the graphene thickness towards those reported for graphite (Paper I).

In the far-infrared and $\mathrm{THz}$ regions, the optical properties of graphene are dominated by the free charge carriers absorption (intra-band). The optical conductivity can be described by a classical Drude-model as discussed in more detail in chapter 3 .

\subsection{Transport properties of graphene}

First transport experiments on graphene (exfoliated graphene on $\mathrm{SiO}_{2} / \mathrm{Si}$ ) have demonstrated high free charge carrier mobility in the range $10000-15000 \mathrm{~cm}^{2} \mathrm{~V}^{-1} \mathrm{~s}^{-1}$ at ambient conditions. Furthermore, graphene exhibits a strong ambipolar field effect due to the linear dependence of the carrier DOS as a function of the energy. The free charge carrier concentration in graphene can be tuned continuously from electrons to holes up to a concentration of $n=10^{13} \mathrm{~cm}^{-2}$, while the mobility parameter is as high as $15000 \mathrm{~cm}^{2} \mathrm{~V}^{-1} \mathrm{~s}^{-1}$, as presented in Fig. 1.8(a) $[6,30]$. This is not the case of high-mobility-semiconductors, for which the mobility is high only for bulk undoped materials and deceases with increasing carrier concentration (see table 1.1).

Magneto-transport measurements realized on exfoliated graphene have provided the first evidence of massless-Dirac fermions in graphene [30, 31]. Fig. 1.8(a) shows the measured cyclotron mass of monolayer graphene as a function of carrier concentration $(n)$ (varied by the field effect). The experimental cyclotron mass data show a square-root dependence with $n$ and the corresponding fit using Eqn. 1.18 gives $v_{f}=1 \times 10^{6} \mathrm{~ms}^{-1}$.

Graphene shows the anomalous quantum Hall effect (QHE) or chiral QHE, which is the shift of the QHE levels by $1 / 2[30,31]$ and it is a direct consequence of the chirality of graphene carriers at the Dirac points (see Fig. 1.8(c)). 
(a)

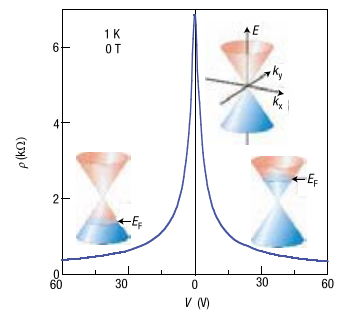

(b)

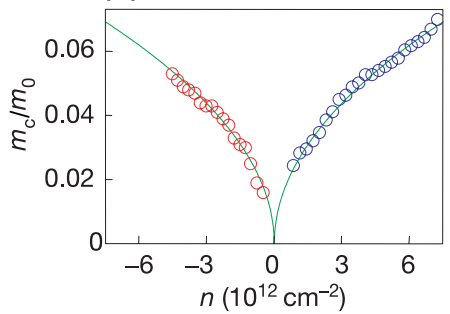

(c)

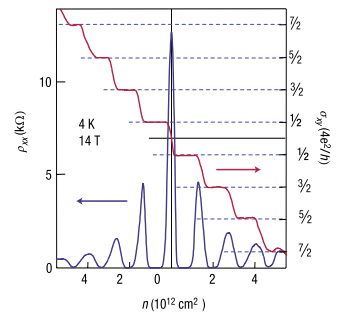

Figure 1.8: (a) Resistivity of graphene as a function of gate bias (Fermi energy), showing a strong ambipolar field effect where carrier concentration can be tuned from holes to electrons. Insets show low-energy band structure of graphene indicating changes in the position of the Fermi energy with changing gate bias. Reprinted with permission from Ref. 6. (b) Cyclotron mass of electrons and holes as a function of their concentrations. Symbols indicates the experimental data and solid curves represent the best fit to theory using Eqn. 1.18. Reprinted with permission from Ref. 30. (c) Chiral quantum Hall effects of monolayer graphene. Reprinted with permission from Ref. 30.

Table 1.1: Properties of high mobility semiconductors [39] and exfoliated graphene on $\mathrm{SiO}_{2}$ at room temperature [6].

\begin{tabular}{lllll}
\hline & $\mathrm{Si}$ & GaAs & InSb & Exfoliated graphene \\
\hline Electron mobility $\left(\mathrm{cm}^{2} \mathrm{~V}^{-1} \mathrm{~s}^{-1}\right)$ & 1417 & 8800 & 77000 & $10000-15000$ \\
Hole mobility $\left(\mathrm{cm}^{2} \mathrm{~V}^{-1} \mathrm{~s}^{-1}\right)$ & 471 & 400 & 850 & $10000-15000$ \\
\hline Band gap $(\mathrm{eV})$ & 1.12 & 1.42 & 0.17 & 0
\end{tabular}

The "half-integer" QHE is considered as the unique fingerprint of high-quality monolayer graphene. Furthermore, graphene is the only material that possesses a room-temperature QHE [32].

Bilayer graphene also possesses high carrier mobility parameters, in the range of $3000-8000 \mathrm{~cm}^{2} \mathrm{~V}^{-1} \mathrm{~s}^{-1}$ at $300 \mathrm{~K}[30,33,34]$, but lower than the mobility of monolayer graphene. Interestingly, a narrow band gap of $250 \mathrm{meV}$ can be created in bilayer graphene, in the presence of a potential difference between the adjacent graphene layers. This can be achieved via the creation of different doping levels between the top and the bottom layers [35] or by application of an electric field perpendicular to the graphene planes [36-38].

\subsection{Graphene and graphite in magnetic field}

When a magnetic field B is applied perpendicularly to the plane of a two-dimensional electron gas (2DEG), the electronic band structure is quantized into discrete 
electronic levels $\left(E_{n}\right)$, so called Landau levels (LLs)

$$
E_{n}=\hbar \omega_{c}\left(n+\frac{1}{2}\right)
$$

where $\omega_{c}=e B / m^{*}$ is the cyclotron frequency, $e$ the electron charge and $n$ the quantum number of the individual LL which is a positive (negative) integer for electron- (hole-) like LLs. Conventional 2DEG semiconductor heterostructures show a linear dependence of the LL energy with the magnetic field and the LLenergies are equally separated. However, graphene shows unique LLs due to its linear band structure at the Dirac point. The LL energy position in graphene are described by [32]

$$
E_{n}=\operatorname{sign}(n) v_{f} \sqrt{e \hbar B|n|}=\operatorname{sign}(n) E_{0} \sqrt{|n|},
$$

with $\mathrm{E}_{0}=v_{f} \sqrt{2 e \hbar B}$.

In contrast to $2 \mathrm{DEG}$, the LLs of graphene show a square-root dependence on the magnetic field and are not equally separated. The fundamental Landau level ( $n=0)$ for graphene is $E_{0}=0$, which can be equally shared by holes and electrons for undoped graphene. The cyclotron mass (in the semi-classical approximation) of graphene can be described by [5]

$$
m_{c}=\frac{1}{2 \pi}\left[\frac{\partial A(E)}{\partial E}\right]_{E=E_{f}},
$$

where $A(E)$ is the area in $k$-space enclosed by the orbit, such as $A(E)=\pi k^{2}=\pi E^{2} / v_{f}^{2}$ and $E_{F}$ is the Fermi energy. The resulting cyclotron mass $m_{c}$ is [5]

$$
m_{c}=\frac{\sqrt{\pi}}{v_{F}} \sqrt{n} .
$$

The LL-energies of bilayer graphene are given by $[26,40,41]$

$$
E_{n}^{\alpha}=\operatorname{sign}(n) \frac{1}{\sqrt{2}} \sqrt{\gamma_{1}^{2}+(2|n|+1) E_{0}^{2}-(1)^{\alpha} \sqrt{\gamma_{1}^{4}+2(2|n|+1) E_{0}^{2} \gamma_{1}^{2}+E_{0}^{4}}} .
$$

Figures 1.9 (a) and (b) show the LL-energies (obtained for $\alpha=1$ ) of monolayer and bilayer graphene, respectively. In contrast to monolayer graphene, the LLenergies of bilayer graphene follow a quasi-linear dependence with the magnetic field.

In the presence of an electromagnetic radiation, the electrons are excited from an occupied landau level state $\mathrm{L}_{n}$ to an unoccupied one $\mathrm{L}_{n}{ }^{\prime}$ by absorbing a photon, 
(a)

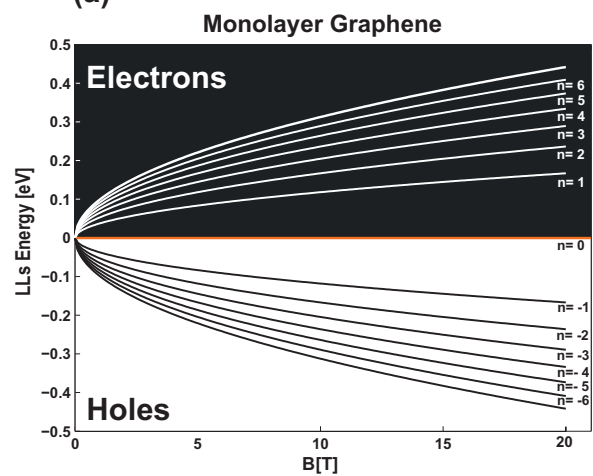

(b)

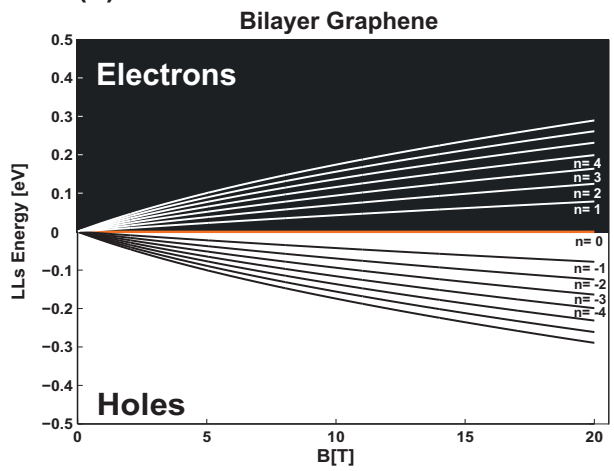

Figure 1.9: (a) Landau level energie as a function of magnetic field for (a) monolayer graphene calculated using Eqn. 1.16 with $v_{f}=1 \times 10^{6} \mathrm{~ms}^{-1}$ and (b) bilayer graphene calculated using Eqn. 1.19 with $v_{f}=1 \times 10^{6} \mathrm{~ms}^{-1}$ and $\gamma_{1}=0.39$.

which is referred to as LL-transitions. The LL-transitions obey the optical selection rules, described for monolayer and bilayer graphene by the relationship

$$
\left|n^{\prime}\right|=|n| \pm 1
$$

The LL-transitions can be classified as following

- The inter-LL-transitions occur between LLs with opposite signs, where an electron is excited from the hole-LL to the electron-LL. The inter-bands LLtransitions energy is given by

$$
E=v_{f} \sqrt{2 e \hbar B}\left(\operatorname{sign}\left(n^{\prime}\right) \sqrt{n^{\prime}}-\operatorname{sign}(n) \sqrt{n}\right)
$$

- The intra-LL-transitions occur between LLs with the same sign, where an electron is excited from electron- (hole-) LL to electron- (hole-) LL.

- The mixture-LLs-transition involves LL-transition of the fundamental level $\left(\mathrm{L}_{0}\right)$. The fundamental-LL is shared by holes and electrons. Consequently, the LL-transitions $\mathrm{L}_{-1} \rightarrow \mathrm{L}_{0}$ and $\mathrm{L}_{0} \rightarrow \mathrm{L}_{1}$ can be considered at the same time as inter- and intra-LL-transition.

Fig. 1.10 is an illustration of LL-energies for graphene at a constant magnetic field presenting the different possible allowed optical inter-, intra- and mixture-LLtransitions, labeled with $*$, \# and $t$, respectively. The symmetric inter- and mixtureLL-transitions with $\left|n^{\prime}\right|=|n| \pm 1$ (for example $\mathrm{L}_{-3} \rightarrow \mathrm{L}_{4}$ and $\mathrm{L}_{-4} \rightarrow \mathrm{L}_{3}$ ) are 
(a)

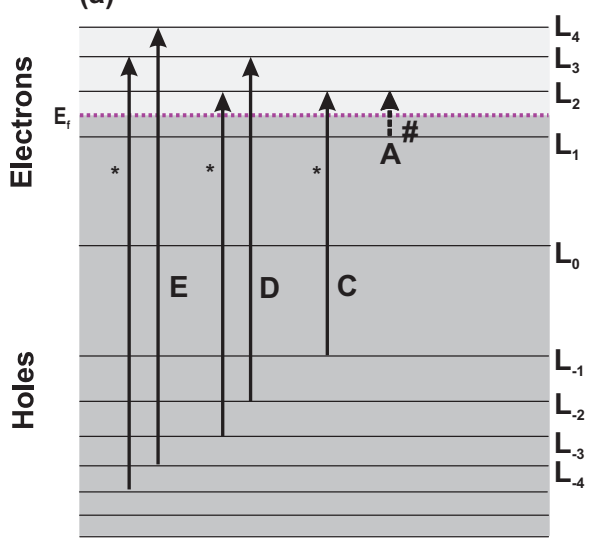

* Inter- band transition \# Intra- band transitions + Mixtre transitions (b)

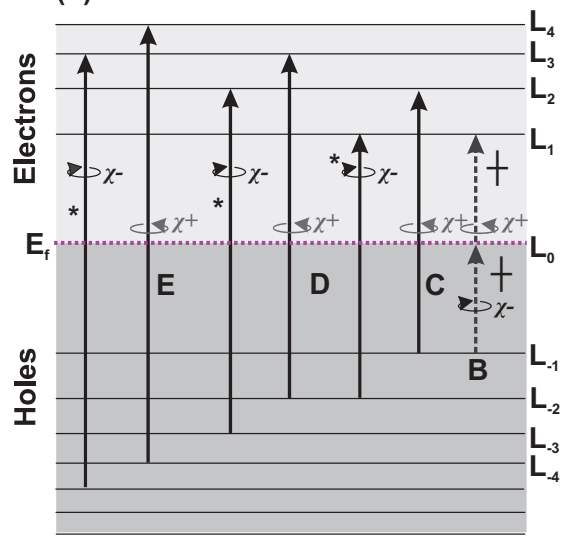

$\succ x^{-}:$: Left handed circular light

$\varangle \chi^{+}$: Right handed circular light

Figure 1.10: Schematic presentation of LL-energies for graphene at a constant magnetic field, the dashed line indicates the Fermi energy and the optically allowed LL-transition-energies are indicated by arrows (a) n-type doped graphene excited with unpolarized light, (b) undoped graphene excited with circularly polarized light. The inter-, intra- and mixture-LL-transition are labeled with $*$, \# and.$+ \chi^{+}$ and $\chi^{-}$denote the right- and left-handed circularly polarized light. Adapted from Ref. 25 .

energetically degenerated (Fig. 1.10 (a) and Eqn. 1.21). As a result, both transitions are excited simultaneously. The separation between the symmetric transitions can be achieved by using circularly polarized radiation [25], as shown in Fig. 1.10(b). 


\section{Chapter 2}

\section{Epitaxial graphene on $\mathrm{SiC}$}

\subsection{Introduction}

Most of the excellent properties of graphene discussed in the previous chapter were demonstrated on exfoliated graphene flakes with limited domain size. The mechanical exfoliation method is a cumbersome preparation method, which makes it unsuitable for large-scale production. Consequently, growth techniques that can produce large-area graphene with properties that match the high-quality of exfoliated graphene are needed in order to implement graphene in future applications. However, obtaining high-quality graphene with well-controlled properties (i.e. thickness, doping, etc.) over large-area remains a significant challenge. Despite the fact that several growth techniques have been developed during the last decade [42], only a few of them could be scaled up to mass production and are applicable at the industrial level. The most promising ways to fabricate large-area graphene are liquid-phase exfoliation of graphite (LPE), chemical vapor deposition of hydrocarbon precursors on transition-metal surfaces (CVD-graphene) and thermal decomposition of silicon carbide $(\mathrm{SiC})$ by sublimation of $\mathrm{Si}$ atoms (see Fig. 2.1).

LPE can produce large quantities of graphene flakes by the oxidation of graphite in liquid environments and sonication to extract individual layers (Fig. 2.1). LPE is a cost-effective growth technique and LPE-graphene can be deposited on a wide range of different substrates. This makes LPE attractive for several applications, such as, graphene ink, coating, composite and transparent conductive electrodes. However, LPE-graphene is defective with a limited lateral size of the flakes, which makes LPE-graphene unsuitable for electronic-based application.

Large-area of high-quality graphene can be grown using CVD and sublimation (Fig. 2.1 and Tbl. 2.1). Graphene can be grown by CVD on a large variety of 
transition metal substrates $(\mathrm{Cu}, \mathrm{Ru}, \mathrm{Ir}, \mathrm{Pt}, \mathrm{Co}, \mathrm{Pd}$, Re, etc.) using hydrocarbon reactants (such as methane). The transition metal substrate plays the role of a catalyst when maintained at high temperatures $\left(400^{\circ} \mathrm{C}-1000^{\circ} \mathrm{C}\right)$, the hydrocarbon reactants are adsorbed at the metal surface and thermally decomposed to carbon species that enable the graphene growth. Large area monolayer graphene (up to 30 inches) is grown by using low pressure-CVD (LP-CVD) on polycrystalline $\mathrm{Cu}$ foil substrate and methane as a hydrocarbon reactant [43]. The as-produced monolayers are polycrystalline where grain boundaries are formed between the adjacent graphene domains. As a result, the carrier mobility is limited and there are large variations of the electronic properties of CVD-graphene, which renders its implementation in electronic devices challenging.

It was shown recently that oxygen-rich $\mathrm{Cu}$ substrate enable the large-area single crystal monolayer graphene growth by CVD up to several square-centimeters $[44,45]$. The single-crystalline monolayer graphene shows high carrier mobility parameters similar to those of exfoliated graphene on silicon oxide substrate (Tab. 2.1). Despite the significant progress, the implementations of CVD-graphene in graphene-based electronics still faces several challenges. The growth rate of single-crystalline CVD graphene is very low, which leads to graphene domain in the centimeter-size range. The control of the size and the shape of singlecrystalline graphene domains over large-area is still a challenge [46]. Besides, most of the electronic and optoelectronic applications require a transfer of CVDgraphene from the metal to a dielectric substrate. During the transfer process, defects (holes, cracks, and wrinkles with lengths of several micrometers), doping and residues are typically introduced into CVD-graphene [47], which can significantly degrade its electronic properties [48]. This transfer process is expensive, thus, limiting the implementation of CVD-graphene at industrial scale. CVD-graphene grown directly on a dielectric substrate has the advantage of avoiding the transfer process, but is far from having the desired crystalline quality and domain size needed for the integration of CVD-graphene in electronic applications [49, 50].

Uniform large-area of high-quality epitaxial graphene (EG) can be grown by sublimation, directly on semi-insulating hexagonal SiC wafers [51-53] without the need of transfer (Fig. 2.1). This is a significant advantage of the sublimation method for graphene-based electronic applications. Several EG-based devices have already been demonstrated, such as high-frequency field effect transistors with a cutoff frequency of $200 \mathrm{GHz}$ [54]. In addition, EG finds application in metrological resistance standards [55]. A schematic summary of the different growth techniques and their main applications is presented in Fig. 2.1. 


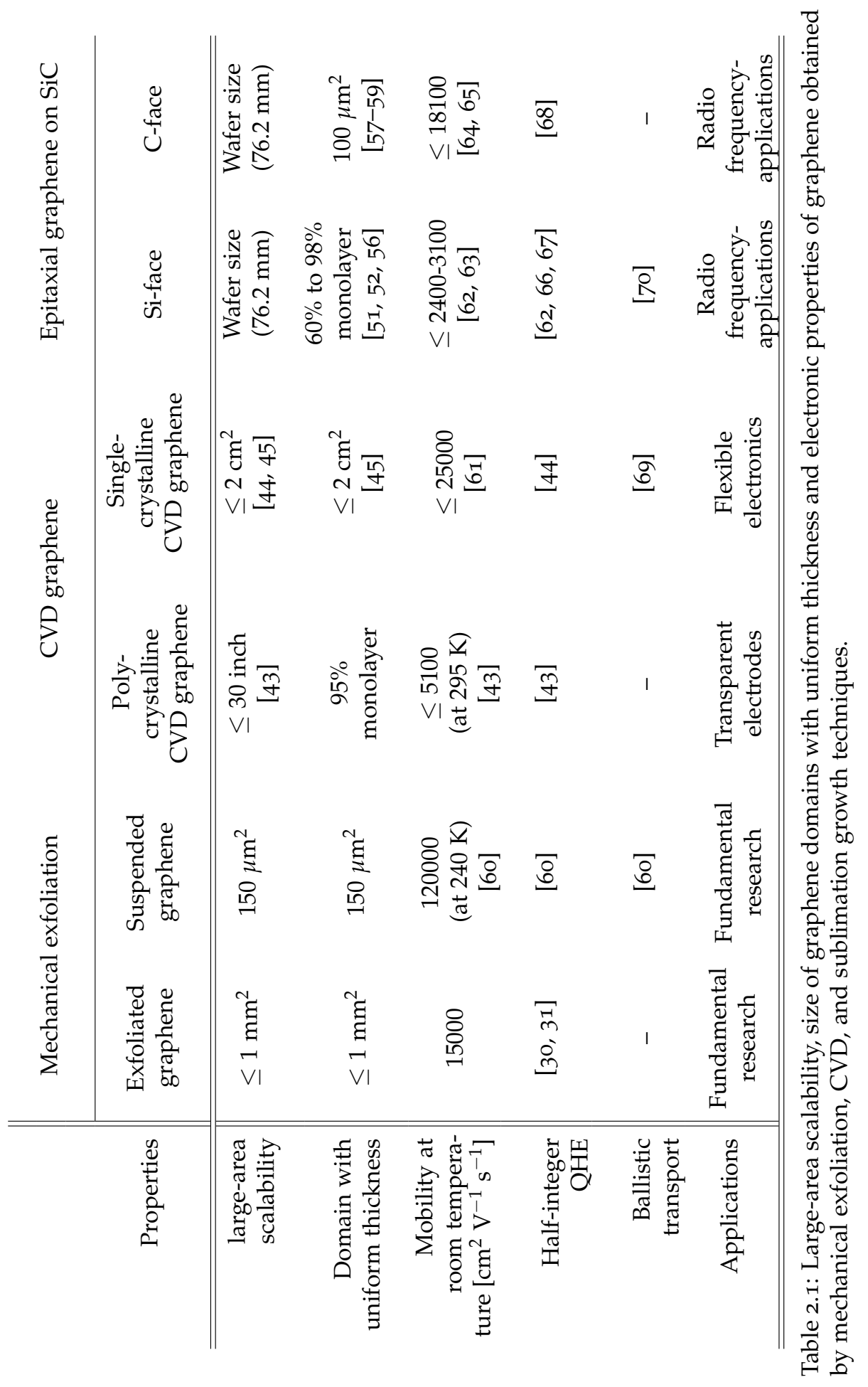




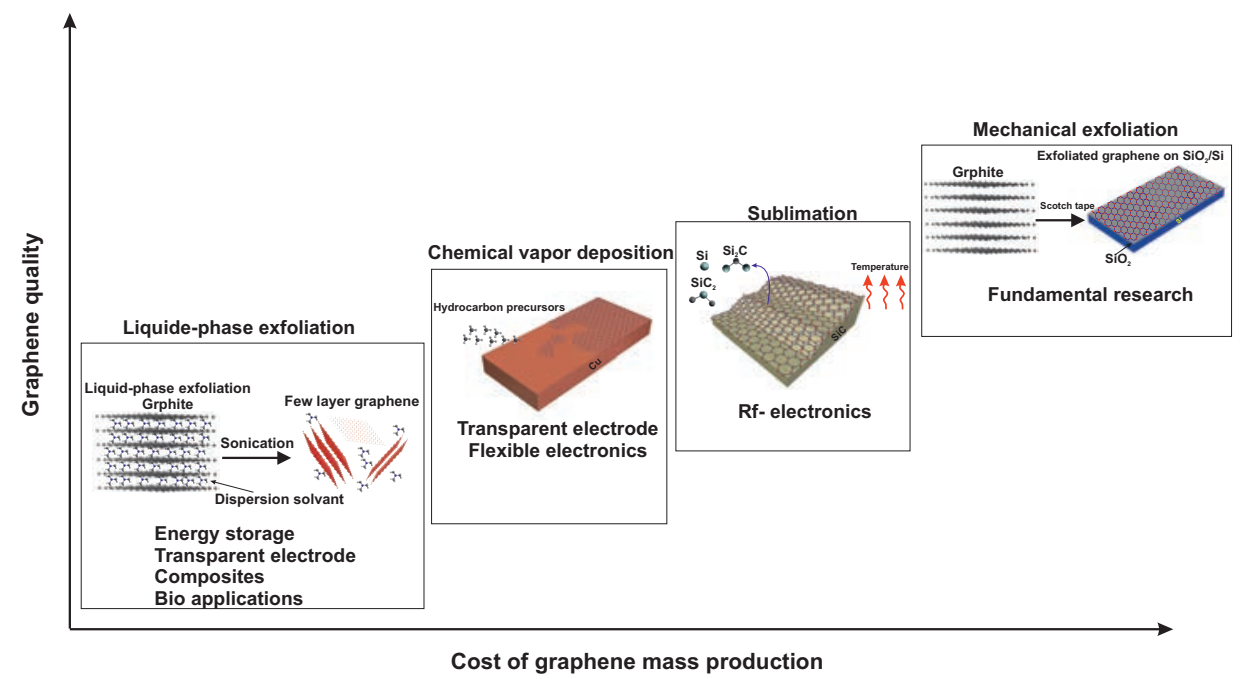

Figure 2.1: Schematic summary of growth techniques used to fabricate large-area graphene.

\subsection{Background of epitaxial graphene on $\mathrm{SiC}$}

The most common used $\mathrm{SiC}$ polytypes for the growth of epitaxial graphene are 3C-SiC (cubic), 4H-SiC (hexagonal) and 6H-SiC (hexagonal). Fig. 2.2(a) shows the different stacking of bilayers of $3 \mathrm{C}-, 4 \mathrm{H}$ - and $6 \mathrm{H}-\mathrm{SiC}$ crystals in the (1120) plane. $3 \mathrm{C}-\mathrm{SiC}, 4 \mathrm{H}-\mathrm{SiC}$, and $6 \mathrm{H}-\mathrm{SiC}$ have bilayers $\mathrm{Si}-\mathrm{C}$ stacking sequence of $\mathrm{ABC} \ldots$, $\mathrm{ABCBABCB} . .$. , and $\mathrm{ABCACBABCACB} .$. , respectively. Hexagonal $\mathrm{SiC}$ is a polar material with two non-equivalent polar faces when cut perpendicularly to the c-axis, where the Si-face corresponds to the (0001) surface and C-face corresponds to the $(000 \overline{1})$ surface. $3 \mathrm{C}-\mathrm{SiC}$ also exhibits similar polar surfaces along the [111] direction.

Graphene can be grown on both $\mathrm{SiC}$ faces using sublimation. In the following, we will refer to epitaxial graphene grown on Si-face and C-face $\mathrm{SiC}(0001)$ as Siface EG and C-face graphene, respectively. The $4 \mathrm{H}$ - and $6 \mathrm{H}-\mathrm{SiC}$ substrate have been the substrate of choice for the growth of EG using sublimation, since their hexagonal surfaces offer an excellent template for EG and they are commercially available (up to $150 \mathrm{~mm}$ for $4 \mathrm{H}-\mathrm{SiC}$ wafers). 3C-SiC has attracted less attention despite the fact that $3 \mathrm{C}-\mathrm{SiC}(111)$ has a hexagonal surface, which can be used also as a template for EG growth. This is due to the difficulty to grow 3C-SiC(111) substrates (or films) with a similar high-quality as $4 \mathrm{H}-\mathrm{SiC}$ (or $6 \mathrm{H}-\mathrm{SiC}$ ) wafers. The surface morphology and the off-cut angle of $\mathrm{SiC}$ also play important roles in the EG formation and properties [72]. 

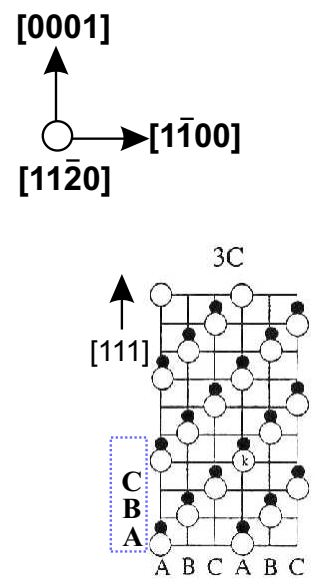

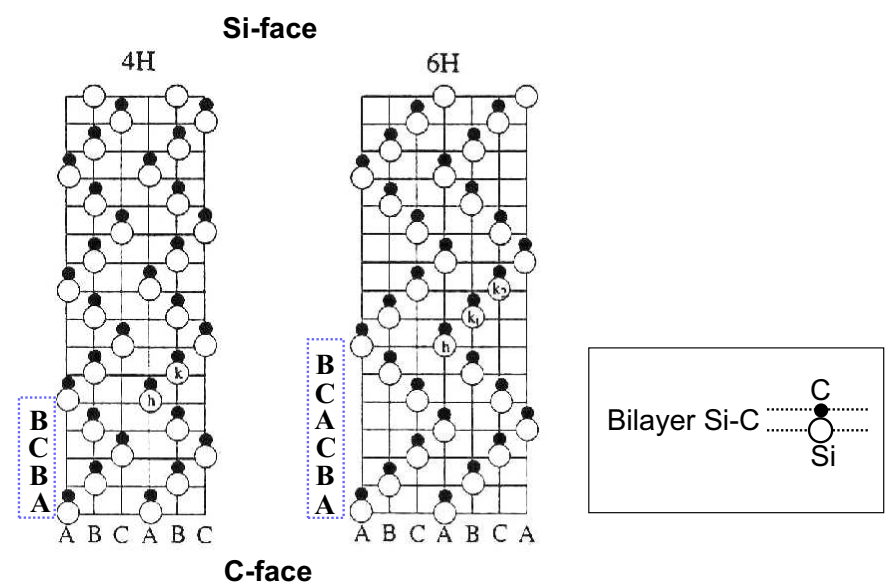

Figure 2.2: Stacking sequence of bilayer $\mathrm{Si}-\mathrm{C}$ of the $3 \mathrm{C}-, 4 \mathrm{H}$ and $6 \mathrm{H}-\mathrm{SiC}$ polytypes in the $(11 \overline{2} 0)$ plane and along the c-axis [0001]. Reprinted with permission from Ref. 71

Sublimation is a thermally driven growth process. When the $\mathrm{SiC}$ substrate is heated above the $\mathrm{Si}$ melting point $\left(1150^{\circ} \mathrm{C}\right)$, the $\mathrm{Si}$ atoms sublime from the $\mathrm{SiC}$ surface (Fig. 2.3(a)). Fig. 2.3(b) shows the vapor pressure of different Si and C species present at the $\mathrm{SiC}$ surface when heated at different temperatures. In an ultra-high-vacuum (UHV) system, graphene can be grown at a temperature as high as $1200^{\circ} \mathrm{C}$, since at this temperature, the Si atoms possess a vapor pressure of $10^{-10}$ bar and can be absorbed by the vacuum system, while the $C$ atoms have a much lower vapor pressure [73] and remain at the surface. When a three bilayer $\mathrm{Si}-\mathrm{C}$ are decomposed from the $\mathrm{SiC}$, the remaining $\mathrm{C}$ atoms reconstruct via $\mathrm{sp}^{2}$ hybridization and form a monolayer EG (Fig. 2.3(a)).

In UHV, the growth of EG occurs far from the thermodynamic equilibrium and the sublimation rate is high, which leads to a poor control of uniformity and thickness. EG grown by UHV-sublimation is defective and shows very rough surface morphology. As a result, the carrier mobility parameters of EG are very low: $\sim 300 \mathrm{~cm}^{2} \mathrm{~V}^{-1} \mathrm{~s}^{-1}\left[{ }^{1} 1\right]$ and $\sim 29 \mathrm{~cm}^{2} \mathrm{~V}^{-1} \mathrm{~s}^{-1}$ [75], for Si-face EG and C-face, respectively. In order to obtain EG graphene with good morphology, uniformity, and electronic properties, several advanced sublimation techniques have been developed. These include: low-temperature $\left(1400^{\circ} \mathrm{C}-1650^{\circ} \mathrm{C}\right)$ sublimation in argon gas (low-temperature sublimation) [51], high-temperature $\left(1800^{\circ} \mathrm{C}-2000^{\circ} \mathrm{C}\right)$ sublimation in argon atmosphere (high-temperature sublimation) [52] and confinement controlled sublimation (CCS) [53]. 
(a)

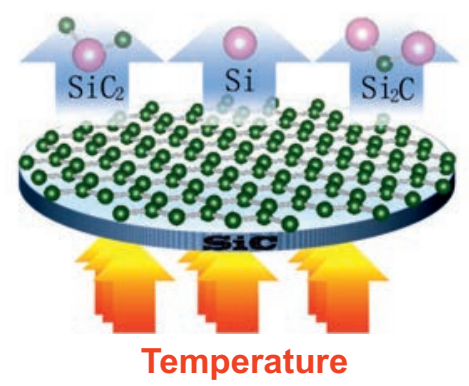

(b)

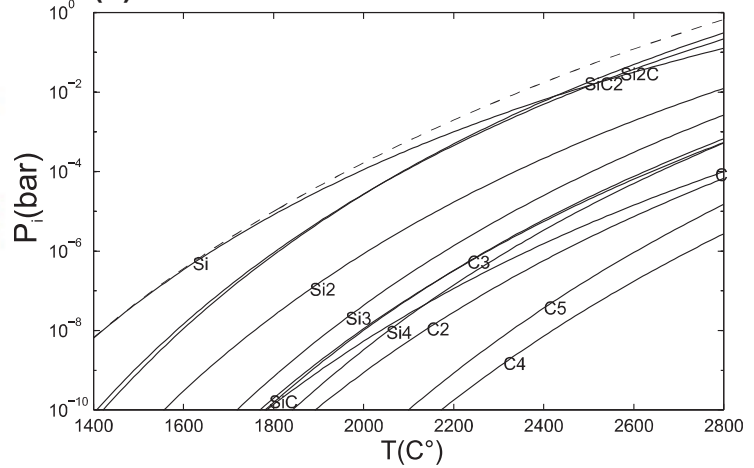

Figure 2.3: (a) Schematic illustration of the sublimation growth method. (b) Equilibrium partial pressures at different temperature shown for volatile $\mathrm{Si}$ species $\left(\mathrm{Si}, \mathrm{Si}_{2}, \mathrm{Si}_{3}, \mathrm{Si}_{2} \mathrm{C}, \mathrm{SiC}\right.$ and $\left.\mathrm{Si}_{3} \mathrm{C}_{3}\right)$ and $\mathrm{C}$ species $\left(\mathrm{C}, \mathrm{C}_{2}, \mathrm{C}_{3}, \mathrm{C}_{2} \mathrm{Si}\right)$ in $\mathrm{SiC}$ system assuming condensed $\mathrm{SiC}$ and carbon. Reprinted with permission from Ref. 74 .

\subsection{EG on Si-face SiC (0001) in UHV}

Fig. 2.4 shows LEED patterns of Si-face $\mathrm{SiC}$ (0001) upon heating. SiC surfaces are usually covered with a native oxide layer $\left(\mathrm{SiO}_{x}\right)$, which has to be removed before the EG growth by annealing the $\mathrm{SiC}$ substrate under a $\mathrm{Si}$ flux at $\geq 800^{\circ} \mathrm{C}$. In this case, the Si-face $\mathrm{SiC}(0001)$ shows Si-rich $(3 \times 3)_{\mathrm{Si}}$, while the C-face $\mathrm{SiC}(000 \overline{1})$ shows a Si-rich $(2 \times 2)_{\mathrm{Si}}$. Note that the native oxide can also be removed by cleaning the $\mathrm{SiC}$ substrate with a hydrofluoric acid solution and by hydrogen etching. In such cases, the $\mathrm{SiC}$ surfaces show $(1 \times 1)$ surface reconstruction. For Si-face $\mathrm{SiC}(0001)$, the thermal annealing of the Si-rich $(3 \times 3)_{\mathrm{Si}}$ surface results in a Crich $(\sqrt{3} \times \sqrt{3})$ R30 (Fig. 2.4(b)). By increasing the annealing temperature up to $1150^{\circ} \mathrm{C}$, a C-rich phase with $(6 \sqrt{3} \times 6 \sqrt{3}) \mathrm{R} 30^{\circ}$ surface reconstruction appears (Fig. 2.4(c)). This surface reconstruction was attributed to the formation of a carbon layer (so-called buffer layer) on top of $\mathrm{SiC}$, rotated by $30^{\circ}$ with respect to the $\mathrm{SiC}$. Further heating (up to $\sim 1280^{\circ} \mathrm{C}$ ) leads to the formation of a new carbon layer between the buffer layer and the SiC. The newly formed carbon layer interacts with the underlying $\mathrm{SiC}$ to form a new buffer layer, while the former buffer layer is detached from the $\mathrm{SiC}$, leading to the formation of the first graphene layer on top with $(1 \times 1)_{\text {graphene }}$ (Fig. $\left.2.4(\mathrm{~d})\right)$. Further heating of Si-face SiC results in the formation of graphite with $(1 \times 1)_{\text {graphite }}$ LEED diffraction pattern (Fig. 2.4(e)).

It was found that Si-face EG has an intrinsic electron doping in UHV environment $\left(\sim 10^{13} \mathrm{~cm}^{-2}\right)$ [76-78]. The buffer layer exhibits $\sigma$-band similar to graphene, indicating that it has a similar atomic arrangement to the one of graphene. How- 
(a)

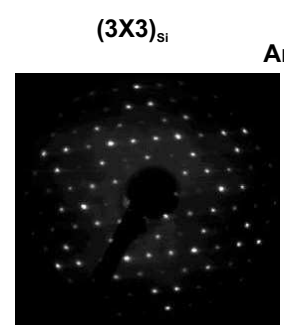

$134 \mathrm{eV}$ (b)

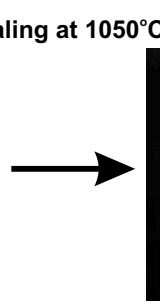

(d)

(c)

$(\sqrt{3} \mathbf{X} \sqrt{3}) \mathrm{R} 30^{\circ}$
$\mathrm{C}^{(6 \sqrt{ } 3 \times 6 \sqrt{3}) \mathrm{R} 30^{\circ}}$

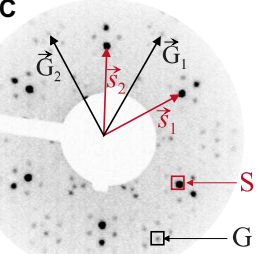

$53 \mathrm{eV}$
$134 \mathrm{eV}$

(e)

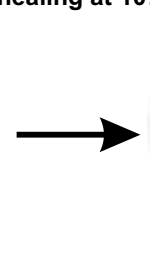

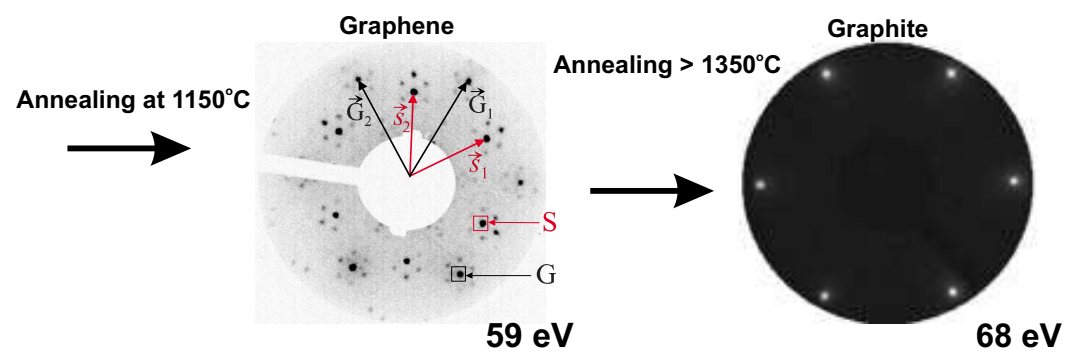

Figure 2.4: LEED patterns of Si-face $\mathrm{SiC}$ (0001) upon annealing in UHV: (a) $(3 \times 3)_{\mathrm{Si}}$, (b) $(\sqrt{3} \times \sqrt{3}) \mathrm{R} 30^{\circ}$, (c) $(6 \sqrt{3} \times 6 \sqrt{3}) \mathrm{R} 30^{\circ}$ surface reconstructions, (d) Si-face EG formation and (e) graphite. The reciprocal lattice vectors of the SiC $\left(S_{1} ; S_{2}\right)$ and graphene $\left(G_{1} ; G_{2}\right)$ lattices are indicated. The LEED patterns in (a) and (b) are adapted from Ref. 81. The LEED in (c), (d) and (e) are adapted from Ref. 79.

ever, the $\pi$-band is distorted due to a strong bonding with the underlying $\mathrm{SiC}$ and thus, the buffer layer shows nonmetallic behavior and different electronic properties compared to graphene [79]. Only one-third of the C-atoms of the buffer layer are covalently bonded to the Si-atoms of the $\mathrm{SiC}$ surface underneath, resulting in a significant degree of $\mathrm{sp}^{3}$ hybridization of the buffer layer. Furthermore, the atomic configuration of the buffer layer leads to a high density of charged Si-atom dangling bonds at the $\mathrm{SiC}$ interface, which introduces a high electron doping into the graphene $[79,80]$.

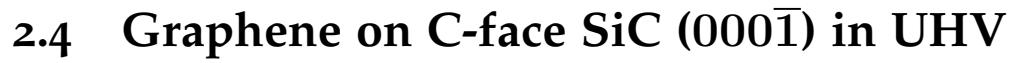

$\mathrm{C}$-face $\mathrm{SiC}$ surface shows a completely different surface reconstruction compared to $\mathrm{Si}$-face $\mathrm{SiC}$, as shown in Fig. 2.5. Heating the Si-rich $(2 \times 2)_{\mathrm{Si}}$ (Fig. 2.4(a)) up to a temperature of $1050^{\circ} \mathrm{C}$ results in the formation of a C-rich $(3 \times 3)_{C} \mathrm{SiC}$ (Fig. 2.3(b)), upon further annealing, the $\mathrm{C}$-rich $(3 \times 3)_{\mathrm{C}} \mathrm{SiC}$ transforms in to a C-rich $(2 \times 2)_{\mathrm{C}}$ 
(a)

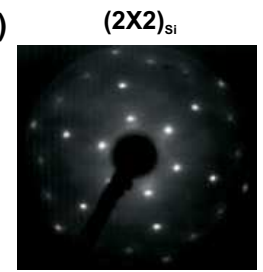

(d)

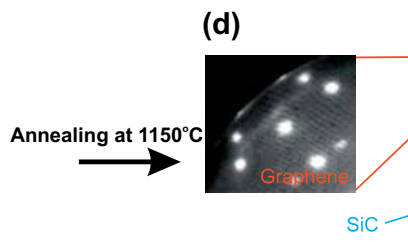

(b)

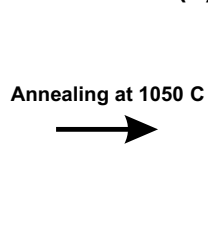

$(2 \times 2)_{c}$ and $(3 \times 3)_{c}$

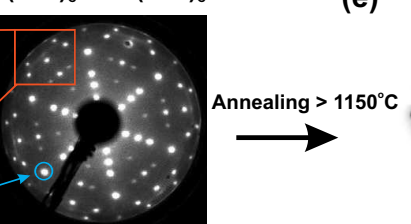

$(3 \times 3)$

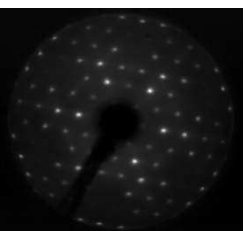

e)
Annealing at $1075 \mathrm{C}$

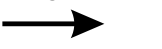

(c)
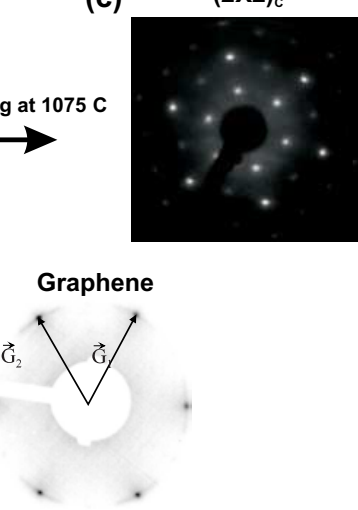

Figure 2.5: LEED patterns C-face $\mathrm{SiC}(000 \overline{1})$ upon annealing of in UHV: (a) $(2 \times 2)_{\mathrm{Si}}$, (b) $(3 \times 3)_{\mathrm{Si}}$, (c) $(2 \times 2)_{\mathrm{C}}$ surface reconstructions, (d) C-face graphene formation on $(2 \times 2)_{C}$ coexisting with $(3 \times 3)_{C}(e)$ FL C-face graphene. The reciprocal lattice vectors of the $\operatorname{SiC}\left(\mathrm{S}_{1} ; \mathrm{S}_{2}\right)$ and graphene $\left(\mathrm{G}_{1} ; \mathrm{G}_{2}\right)$ lattices are indicated. The LEED patterns are adapted from Refs. 79 and 81.

phase (Fig. 2.5(c)). By further increasing the annealing temperature up to $1150^{\circ} \mathrm{C}$, C-face graphene is formed (Fig. 2.4(d)) suggesting the growth rate of C-face graphene is much higher compared to Si-face EG. Furthermore, it was found that the $(2 \times 2)_{C}$ reconstructioncoexists with $(3 \times 3)_{C}$ before graphene formation $[79,82]$ and C-face graphene grows on top of C-rich $(2 \times 2)_{C}$ or $(3 \times 3)_{C}$. The ratio between the two reconstructions depends on the growth temperature, surface preparation, heating furnaces, etc.

Further heating results in the formation of few layers (FL) C-face graphene. Unlike Si-face SiC $(0001)$ that shows single $(1 \times 1)_{\text {graphene, }}$ FL C-face LEED pattern shows multiple $(1 \times 1)_{\text {graphene }}$ diffraction spots and a diffusive ring-like diffraction pattern with a strong intensity modulation (Fig. 2.5(d) and (e)). These diffraction patterns was attributed to the rotation of adjacent graphene layers in the stack, exhibiting preferential rotational angles. FL C-face graphene shows a Dirac-like band structure similarly to single layer graphene [79]. This observation indicates that the adjacent layers in FL C-face graphene stack are electronically decoupled and behave as a stack of non-interacting graphene layers. Furthermore, it was shown that $\mathrm{C}$-face graphene interacts weakly with the underlying $\mathrm{SiC}$ substrate and a buffer layer is absent, in contrast to Si-face EG [79]. 
(a)

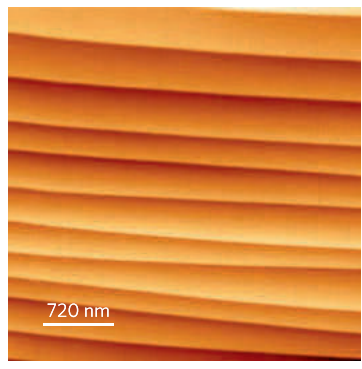

(d)

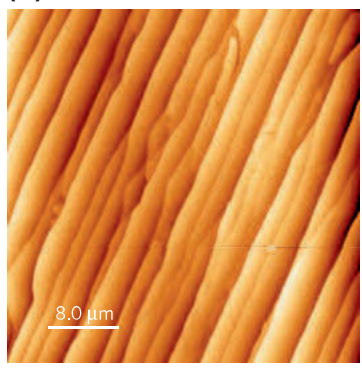

(b)

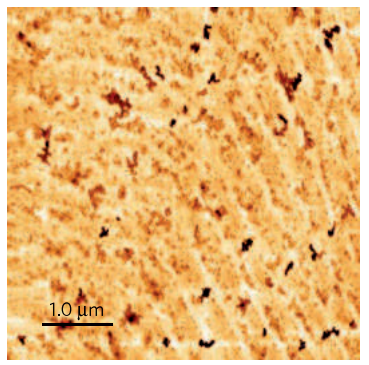

(e)

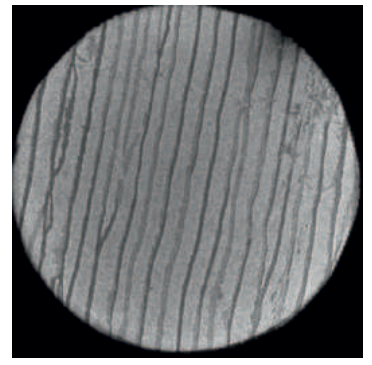

(c)

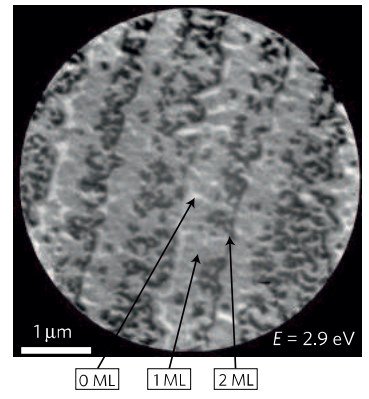

(f)

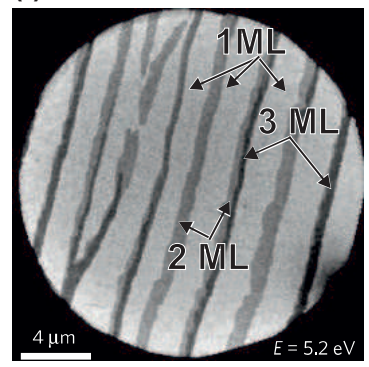

Figure 2.6: (a) AFM image of Si-face $\mathrm{SiC}$ (0001) after hydrogen etching. (b) and (c) AFM and LEEM images of Si-face EG grown by UHV-sublimation. (d) AFM and (e) LEEM images of Si-face EG grown by low-temperature sublimation in argon atmosphere, receptively. (f) A zoomed LEEM image of Si-face EG grown by low-temperature sublimation in argon atmosphere. The LEEM image shows the formation of bilayer and trilayer Si-face EG around the step edges. Images are reprinted with permission from Ref. 51 .

\subsection{Low-temperature EG on Si-face SiC (ooo1)}

Emtsev et. al. [51] have demonstrated uniform large-area monolayer (ML) Si-face EG by sublimation in argon atmosphere (Fig. 2.6(e)), with pressure of 1000 mbar and a growth temperature of about $1650^{\circ} \mathrm{C}$. The presence of a dense argon gas slows down the Si atoms evaporation by acting as a diffusion barrier and shifting the $\mathrm{Si}$ atoms desorption to a significantly high temperature (up to $1500^{\circ} \mathrm{C}$ ). This opens the temperature window where the $\mathrm{SiC}$ surface undergoes a surface restructuring before graphene is formed, leading to a major improvement of the Si-face EG surface morphology, as shown in Fig. 2.6(d). Furthermore, the hightemperature increases the diffusion rate of the carbon atoms, which improves EG crystalline quality.

LEEM investigations (Fig. 2.6(f)) show that using this method uniform ML graphene can be formed along the $\mathrm{SiC}$ terraces. However, bilayer and trilayer 
graphene are formed close to the step edges. Often for low-temperature sublimation in argon, prior to graphene growth, the $\mathrm{SiC}$ surface is treated in hydrogen at relatively high-temperatures to remove the mechanically polishing process damage. Such so-called hydrogen etching results into a significant step bunching, as well as a change of the initial $\mathrm{SiC}$ from relatively flat surface (with atomic terrace steps height) to well-defined terraces perpendicular to the [0001] direction (with relatively higher terrace step height) $[83,84]$ (Fig. 2.6(a)). Additionally, during the initial stage of graphene growth, another significant step bunching occurs where a higher macro-terrace steps height with larger terraces in between are formed [85] (Fig. 2.6(d)). It was found that Si-face EG nucleates and exhibits much higher growth rate at the macro-steps compared to the terraces [86-88]. Si-face EG grown by low-temperature sublimation in argon forms on top of the buffer layer and exhibits high electron doping, similarly to the case of Si-face EG grown by UHV-sublimation [51].

Si-face EG grown by low-temperature sublimation in argon shows a higher carrier mobility of $900 \mathrm{~cm}^{2} \mathrm{~V}^{-1} \mathrm{~s}^{-1}$ at $300 \mathrm{~K}$ compared to Si-face grown in UHV with a mobility of $300 \mathrm{~cm}^{2} \mathrm{~V}^{-1} \mathrm{~s}^{-1}$ at $300 \mathrm{~K}$. Similarly, Tedesco et. al. also reported the improvement of carrier mobility of Si-face EG grown in argon compared to those grown in an intermediate vacuum [89], as shown in Fig. 2.7.

\subsection{Low-temperature graphene on C-face $\operatorname{SiC}(000 \overline{1})$}

Confinement controlled sublimation (CCS) is another approach used to improve the uniformity of EG by confining $\mathrm{SiC}$ substrate in an enclosure (in argon or vacuum) with an orifice on top, which limits the Si escape and increases the Si vapor pressure at the $\mathrm{SiC}$ surface. This keeps the process close to the thermodynamic equilibrium. The high $\mathrm{Si}$ pressure at the $\mathrm{SiC}$ surface allows to grow graphene in an intermediate vacuum $\left(10^{-7}\right.$ to $\left.10^{-4} \mathrm{mbar}\right)$ or in moderate argon pressure [53]. The graphene growth temperature in CCS ranges from 1300 to $1600^{\circ} \mathrm{C}$.

In contrast to Si-face EG, C-face graphene was found to form typically multilayer or FL graphene (Fig. 2.8(a)). Fig. 2.8(b) shows atomic force microscopy (AFM) topographic image of CCS grown multilayer graphene, revealing well-defined $\mathrm{SiC}$ terraces unlike C-face graphene grown in vacuum. Moreover, C-face graphene grown by CCS exhibits wrinkles (white lines in Fig. 2.8(b)), which may originate from compressive thermal strain generated by the different thermal expansion coefficients of $\mathrm{SiC}$ and graphene [9o]. Similar to the case of UHV, C-face graphene grown by CCS shows multiple $(1 \times 1)_{\text {graphene }}$ spots. One set of $(1 \times 1)_{\text {graphene }}$ spots rotated by $30^{\circ}(\mathrm{R} 30)$ with respect to the $(1 \times 1)$ diffraction spots of the SiC. There 


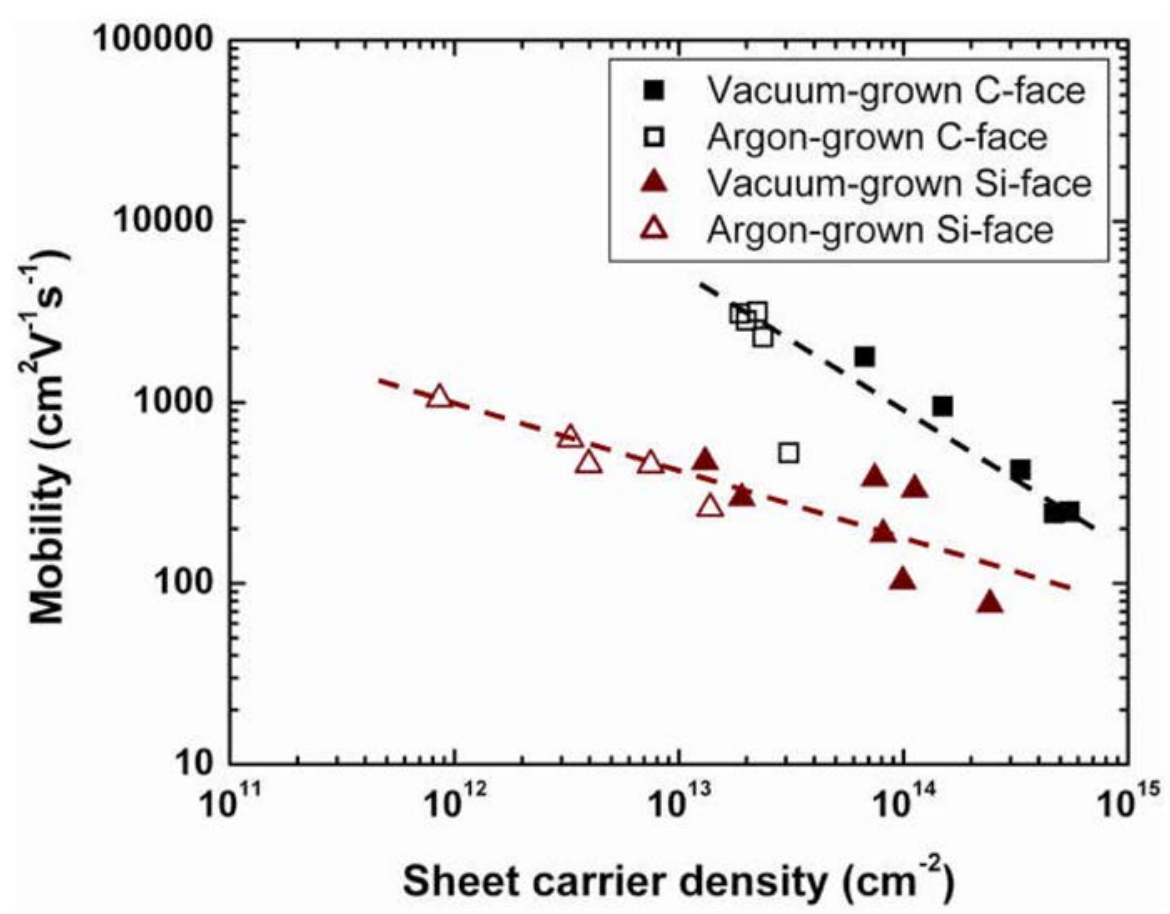

Figure 2.7: Hall effect mobility versus sheet carrier density measured at $300 \mathrm{~K}$ for $\mathrm{C}$-face and Si-face grown in vacuum and in argon. The dashed lines are linear fits to the data for C- and Si-face samples. Reproduced with permission from Ref. 89.

is also a diffusive ring located at $0 \pm 2.2^{\circ}\left(\mathrm{R}^{2 \pm}\right)$ with respect to the $\mathrm{SiC}$ diffraction spots (see Fig. 2.8(c)). Multilayer C-face graphene grown by CCS was shown to have a Dirac-like band structure, (see Fig. 2.8(d)) [91] but also $A B$-stacking bilayer and multilayer arrangement $[41,92,93]$. The presence of a Dirac-like band structure indicates that the individual layers in the FL C-face graphene stack are electronically decoupled and the stack behaves as a single layer graphene. The origin of the decoupling was attributed to the rotation disorder between the adjacent graphene layers [15]. The decoupling of FL C-face graphene was further confirmed by infrared transmission in magnetic field that showed a square-root dependance of the LL-transitions energies on magnetic field [97, 98]. It was found that the first graphene layers close to the SiC (0001) substrate possess a highelectron doping $\left(\sim 9.2 \times 10^{12} \mathrm{~cm}^{-2}\right)$, the top graphene layers are gradually doped until reaching the undoped graphene layers on the top [96, 99]. The doping of the graphene layer close to the $\mathrm{SiC}(000 \overline{1})$ was attributed to a charge transfer from the interface and the $\mathrm{SiC}(000 \overline{1})$ substrate. C-face graphene grown by CCS 
(a)

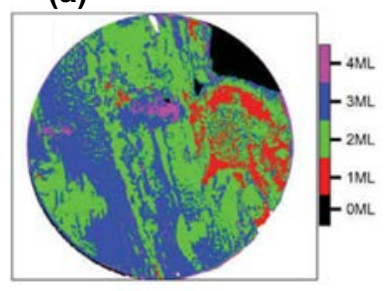

(b)

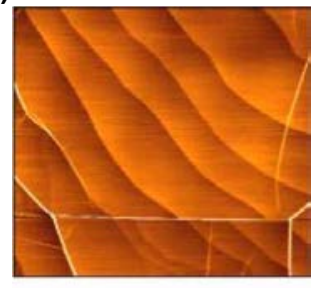

(c)

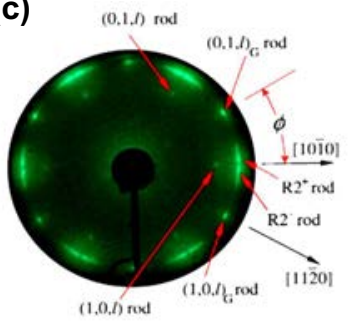

(d)

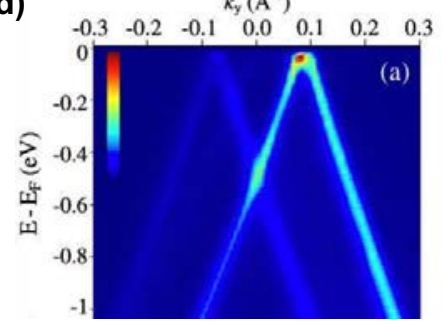

(e)

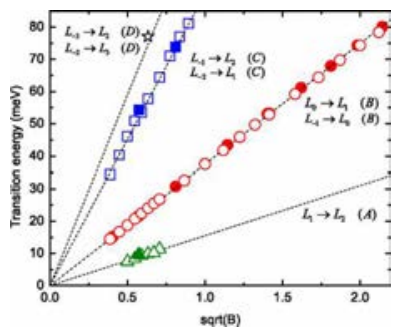

Figure 2.8: (a) Thickness map of CCS FL C-face graphene extracted from LEEM measurement. The field of view is $10 \mu \mathrm{m}$. Reprinted with permission from Ref. 94. (b) AFM images of CCS grown multilayer C-face graphene (10 12 ML). Reprinted with permission from Ref. 95. (c) LEED patterns of CCS multilayer $\mathrm{C}$-face graphene $(\sim 10 \mathrm{ML})$ acquired at $72.2 \mathrm{eV}$, showing the graphene and $\mathrm{SiC}$ spots and the diffuse arcs labeled as the $\mathrm{R}^{2+}$ and $\mathrm{R}^{2-}$ rods. Reprinted with permission from Ref. 95. (d) ARPES measured from the top 3 layers of a 10-layer CCS grown multilayer graphene C-face graphene film. Adapted from Ref. 96. (e) Inter-LL-transitions energies versus the square-root of the magnetic field. The solid symbols are data obtained for the CCS FL C-face graphene sample tilted with respect to the direction of $\mathrm{B}$ by an angle of $50^{\circ}$. The dashed lines represent calculated transition energies for single layer graphene with Fermi velocities $v_{f}=1.03 \times 10^{6} \mathrm{~ms}^{-1}$ [97].

method shows a high carrier mobility at room temperature, in the range of 5000 to $15000 \mathrm{~cm}^{2} \mathrm{~V}^{-1} \mathrm{~s}^{-1}[53,100-102]$. This was attributed to the high-quality of graphene, the decoupling of the adjacent graphene layers and the weak interaction between C-face graphene and SiC. Despite that, half-integer QHE was not observed for FL C-face graphene. On the other hand, For monolayer C-face graphene grown by CCS, half-integer QHE was observed and a very high carrier mobility of 15000 $\mathrm{cm}^{2} \mathrm{~V}^{-1} \mathrm{~s}^{-1}$ and an electron density of $1.27 \times 10^{12} \mathrm{~cm}^{-2}$ was found [103].

$\mathrm{C}$-face graphene also can be grown by sublimation in argon environment in CVD-type reactor. Typical growth temperatures are in the range of $1500-1600^{\circ} \mathrm{C}$ and typical argon pressures range from 50 to 1000 mbar [104]. Similar to the CCS growth method, C-face graphene in this case consists also of small domains with different thicknesses. The surface defects of $\mathrm{SiC}$ were found to play an impor- 
(a)

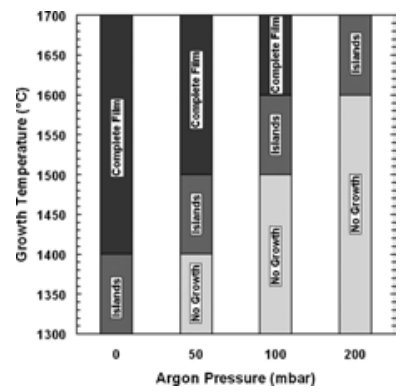

(b)

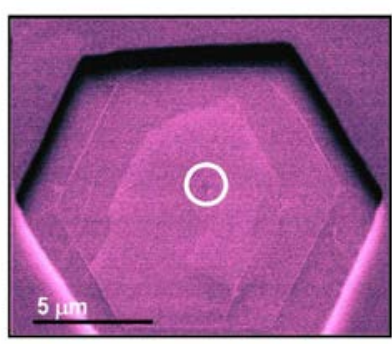

(c)

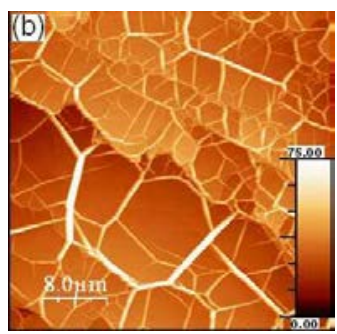

Figure 2.9: (a) Growth mode of C-face graphene as a function of growth temperature and argon growth pressure. Zero argon pressure corresponds to an in-vacuum growth at $10^{-5}$ mbar. Reprinted with permission from Ref. 104. (b) Electron channeling contrast imaging microscopy image showing the nucleation of $\mathrm{C}$-face graphene at a threading screw dislocation, indicated with the white circle. Reprinted with permission from Ref. 105. (c) Typical AFM images of Cface graphene grown by low-temperature sublimation. Reprinted with permission from Ref. 89 .

tant role in the growth in this case. Fig. 2.9(a) shows a histogram of the growth mode of C-face graphene as a function of growth temperature and argon growth pressure [104]. It is seen that graphene islands are typically formed before the entire $\mathrm{SiC}$ is covered with graphene, indicating that the C-face graphene grows in a three-dimensional mode. Hite et. al. showed that graphene nucleates around threading screw dislocation defects forming thick islands (see Fig. 2.9(b)). As the sublimation process proceeds, graphene islands coalesce, which results in non homogeneous FL or multilayer C-face graphene with a rough surface morphology [106] (Fig. 2.9(c)). Camara et. al. have investigated the stacking order of FL C-face graphene grown around the screw dislocations in intermediate vacuum regime and found that graphene is composed of $A B$-stacked multilayer (i.e. graphite) [107].

The transport properties of C-face graphene grown by low-temperature sublimation in argon were investigated by infrared transmission in magnetic field [108] and mid-infrared optical Hall effect [109]. These investigations showed inter-LLtransitions energies with square-root and sub-linear dependance on the magnetic field (Fig. 2.7, [109]). The quasi-linear dependance indicates that C-face graphene contains $A B$-stacked bi- and tri-layer graphene. The electric magneto-transport measurements showed further that $\mathrm{C}$-face graphene grown by low-temperature sublimation in argon exhibits a higher carrier mobility $\left(3000-5000 \mathrm{~cm}^{2} \mathrm{~V}^{-1} \mathrm{~s}^{-1}\right.$ at room temperature and hole doping [89]) compared to graphene grown by sublimation in vacuum, as shown in Fig. 2.7. 
(a)

(b)

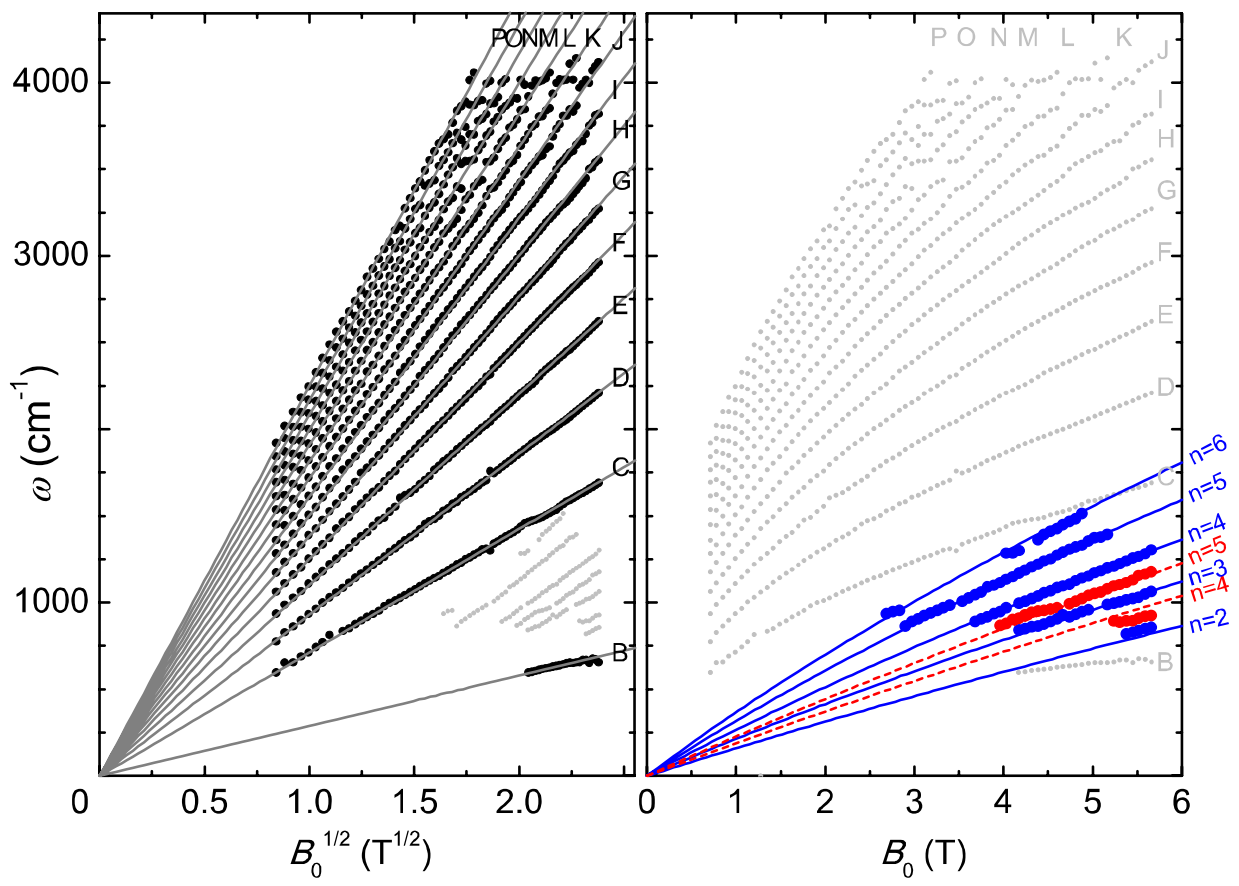

Figure 2.10: Inter-Landau-level transition energies in multilayer (10-20 MLs) Cface graphene on $6 \mathrm{H}-\mathrm{SiC}$ grown at $1400{ }^{\circ} \mathrm{C}$ in argon atmosphere determined from optical Hall effect measurements at $1.5 \mathrm{~K}$. Solid lines denote single-layer graphene [(a) black solid lines and dots], bilayer graphene [(b) blue solid lines and dots], and trilayer graphene [(b) red dashed lines and dots] dependencies on the magnetic field. Reprinted with permission from Ref. 109.

The interface properties of C-face graphene grown by low-temperature in argon pressure are not well understood. Luxmi et. al. observed the formation of diffraction spots with $(\sqrt{3} \times \sqrt{3})$ R30 $0^{\circ}$ instead of the $(1 \times 1) \mathrm{SiC}$ spots for C-face graphene grown at $1600^{\circ} \mathrm{C}$ and argon pressure of $1000 \mathrm{mbar}$ [110]. They explained this observation by the presence of a silicate layer $\left(\mathrm{Si}_{2} \mathrm{O}_{3}\right)$ at the interface between $\mathrm{C}$-face graphene and $\mathrm{SiC}$. It was found that the silicate layer suppresses the growth of uniform C-face graphene. The origin of the interface layer was attributed to unintentional oxidation of the $\mathrm{C}$-face $\mathrm{SiC}$ during the annealing process in argon, due to the presence of oxygen species in the growth reactor. The silicate layer was reported to form at a temperature of $\approx 1200^{\circ} \mathrm{C}$ and to remain stable up to $1640^{\circ} \mathrm{C}$. Transmission electron microscopy studies showed that C-face graphene can either form on top of an amorphous interface layer composed of Si and C atoms [111113], or grow directly on the C-face $\mathrm{SiC}$ without an interface layer [114, 115]. 
(a)

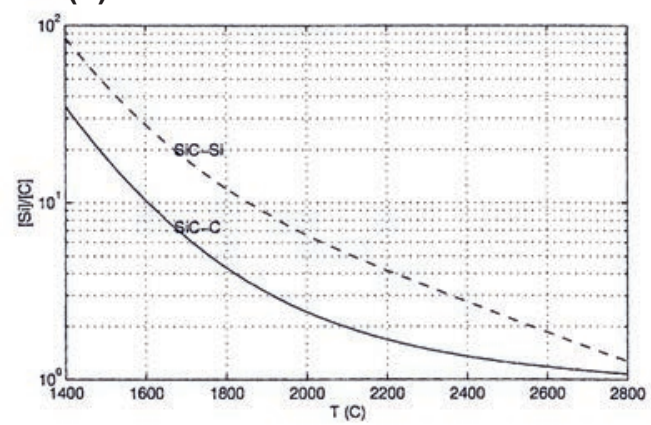

(b)

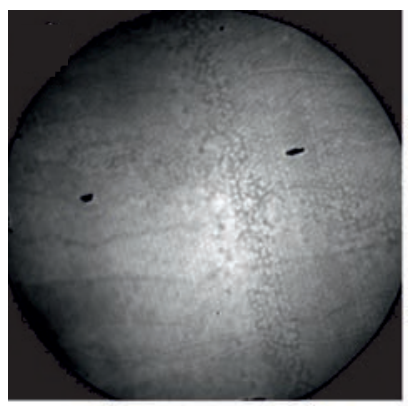

Figure 2.11: (a) The $\mathrm{Si} / \mathrm{C}$ ratio in the vapor phase as a function of annealing temperature of $\mathrm{SiC}$ in C-rich (solid line) and Si-rich environment (dashed-line) (reproduced with permission from Ref. 116). (b) LEEM images taken from monolayer Si-face EG grown on $0.03^{\circ} 6 \mathrm{H}-\mathrm{SiC}(0001)$, the field of view is $50 \mu \mathrm{m}$. The graphene sample was a temperature of $2000^{\circ} \mathrm{C}$ at an ambient argon pressure of 1000 mbar. Adapted from Ref. 117.

\subsection{High-temperature EG on Si-face $\mathrm{SiC}(0001)$}

A novel growth method was developed by Yakimova et. al. [52, 118] for large-area homogenous Si-face EG by using a high-temperature sublimation in argon atmosphere. This method uses high growth temperatures from $1800^{\circ} \mathrm{C}$ to $2000^{\circ} \mathrm{C}$. The growth of Si-face EG is performed inside a high purity dense graphite crucible with excellent isothermal conditions to ensure uniform temperature distributions. The crucible is placed in radio frequency inductively heated furnace. The hightemperature and the growth inside a high purity graphite crucible present significant advantages for achieving large-area of uniform and high-quality Si-face EG. Fig. 2.11 (a) shows the $\mathrm{Si} / \mathrm{C}$ ratio in the vapor phase as a function of annealing temperature of $\mathrm{SiC}$ in $\mathrm{C}$-rich (solid line) and Si-rich environments (dashed line). The growth at high-temperature and the $\mathrm{C}$-rich environment leads to the decrease of the $\mathrm{Si} / \mathrm{C}$ ratio, consequently more carbon atoms are available for the growth of Si-face EG. Contrary to the low-temperature sublimation, in which hydrogen etching is performed to prepare uniform SiC terraces before graphene growth, high-temperature sublimation uses in-situ sublimation etching in argon for: i) controlling the surface restructuring (step bunching), and ii) obtaining regular terrace structures and controlled terraces step height before the graphene formation. This procedure is achieved by optimization of the growth process parameters and substrate misorientation (off-cut angle). Large-area of continuous monolayer Siface EG without any bilayer neither multilayer inclusions at the terraces steps 
(a)

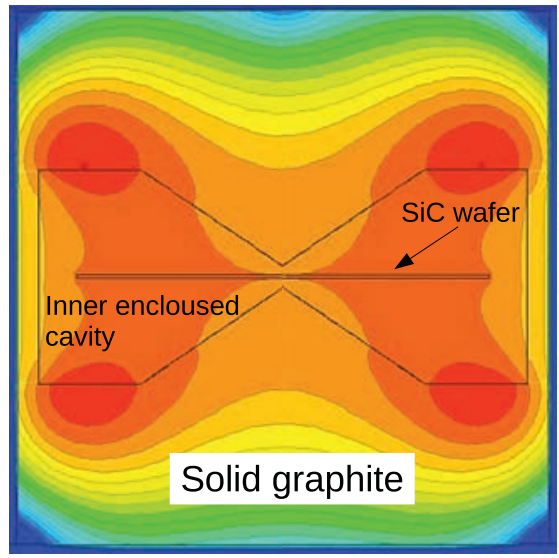

(b)

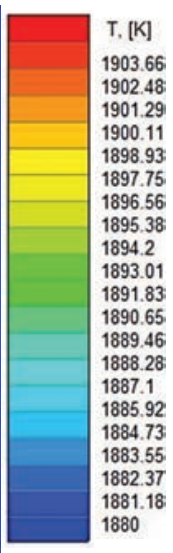

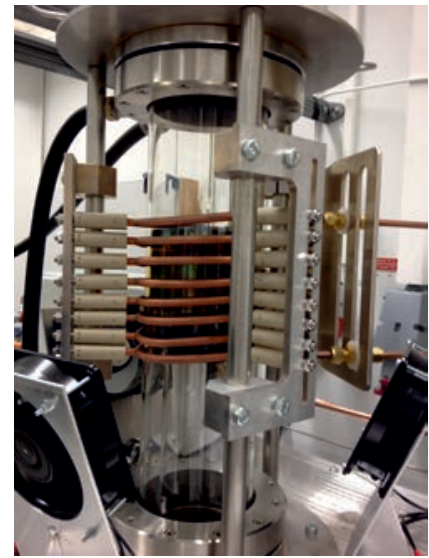

Figure 2.12: (a) Temperature distribution inside the growth reactor and the crucible. Adapted from Paper VII. (b) Image of the growth reactor used for the growth of Si-face EG samples (Courtesy of Prof. Rositsa Yakimova).

have been demonstrated on nominally on-axis $6 \mathrm{H}-\mathrm{SiC}(0001)$ with an off-cut $0.03^{\circ}$ over an area of $\sim 2000 \mu \mathrm{m}^{2}$ (Fig. 2.11 (b)) [117]. These monolayers grew on $6 \mu \mathrm{m}$ wide terraces and 1-1.5 $\mathrm{nm}$ terraces step height (Fig. 2.11 (b)). Si-face EG grown by high-temperature sublimation also exhibits a buffer layer [52] and similar structural properties as Si-face EG grown by low-temperature sublimation in argon or UHV-sublimation [52]. Thus, Si-face EG has universal structural properties (buffer layer and electron doping) independently of the growth conditions. The Si-face EG grown by high-temperature sublimation in argon shows a high crystalline quality, in addition to good uniformity across the terraces steps, the observation of half-integer QHE, as well as a high mobility $\sim 2400 \mathrm{~cm}^{2} \mathrm{~V}^{-1} \mathrm{~s}^{-1}$ at room temperature for typical electron doping in the range $5 \times 10^{11}$ to $1 \times 10^{12} \mathrm{~cm}^{-2}$ (at room temperature) [62].

In this thesis, we have developed a new crucible to achieve large-area of uniform Si-face EG over 2 inch $\mathrm{SiC}$ substrates. To ensure uniform temperatures distribution, we have modeled the heat transfer in the growth system and temperature distribution of the crucible using virtual reactor software from Soft-Impact, Ltd. Fig. 2.12(a) shows the design and the thermal distribution of the crucible with a uniform temperatures distribution (Paper VII). The free charge carrier mobility parameters in Si-face EG grown with the new crucible were determined to be in the range $1500-2500 \mathrm{~cm}^{2} \mathrm{~V}^{-1} \mathrm{~s}^{-1}$ (Papers VI and VII). 


\subsection{High-temperature graphene on C-face $\operatorname{SiC}(000 \overline{1})$}

In similarity to the other sublimation methods, the growth of uniform large-area $\mathrm{C}$-face graphene by high-temperature sublimation in argon is also very challenging. The C-face graphene obtained by this growth method consists of relatively small domains with a size of a few microns. Johansson et. al. have investigated the stacking of FL C-face graphene grown by high-temperature sublimation in argon using selected area LEED (micro-LEED). They found that FL C-face graphene shows no rotational disorder between the adjacent graphene layers in the stack,

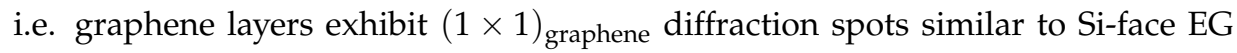
(Fig. 2.13(b) and (c)), and that the rotation of different domains does not occur between adjacent layers in the stack but rather in-plane within the same graphene sheet (Fig. 2.13(d)) [119, 120]. Recently, the authors reported that FL C-face graphene is $A B$-stacked (Fig. 2.13(f)) and the single $\pi$-band observed in ARPES experiments is a superposition of several multiple bands associated with two, three or more $A B$-stacked layers (Fig. 2.13(e)), in contrast to the results reported for C-face graphene grown by other sublimation techniques [120]. Johansson et. al. could not identify any interface between C-face graphene and $\mathrm{SiC}[119,121]$. On the contrary, in this thesis, we have identified an amorphous interface layer between $\mathrm{C}$-face graphene and $4 \mathrm{H}-\mathrm{SiC}(000 \overline{1})$ in relation with polishing defects using highresolution transmission electron microscopy and spectroscopic ellipsometry. We have found that the interface layer is composed of $\mathrm{Si}, \mathrm{C}$ and $\mathrm{O}$ atoms. We also have found that the interface layer thickness increases with increasing the growth temperature [122]. Our results were later confirmed by Nicotra et. al.. In paper II, we have identified a $\mathrm{SiO}_{x}$ layer between graphene and $4 \mathrm{H}-\mathrm{SiC}(\mathbf{0 0 0} \overline{\mathbf{1}})$ without polishing defects using selected area X-ray photoelectron spectroscopy and we have shown that its chemical composition changes towards $\mathrm{SiO}_{2}$ and its thickness increases with aging in ambient conditions. The interface $\mathrm{SiO}_{x}$ layer also causes the formation of non-ideal Schottky contact between the ML C-face graphene and SiC. In this thesis, we investigated the interaction between the individual layers in FL $\mathrm{C}$-face graphene stack using mid-infrared optical Hall effect and the stacking order of FL C-face graphene using micro-LEED. We found that C-face graphene consists of decoupled non-interacting sheets independently of the substrate polytype. We further determined that FL C-face graphene does not show any rotational disorder. However, the presence of polishing defects on the substrate surface may cause the formation of $A B$-stacked inclusions. This may provide a possible explanation for the finding of $A B$-stacked FLG reported by Johansson et. al. [120]. 
(a)

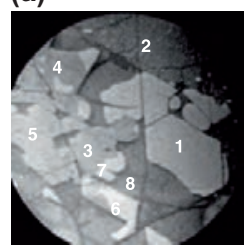

(e)

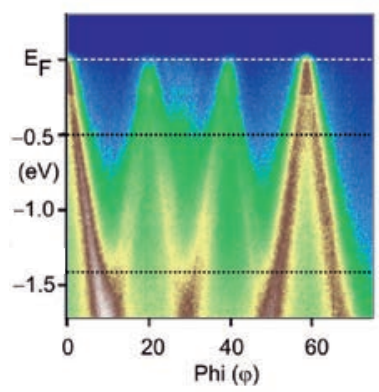

(b)

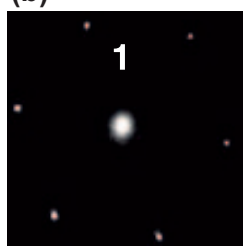

(c)

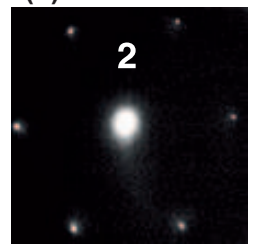

(d)

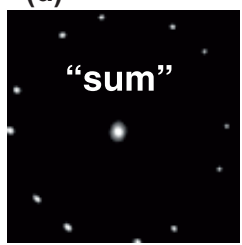

(f)

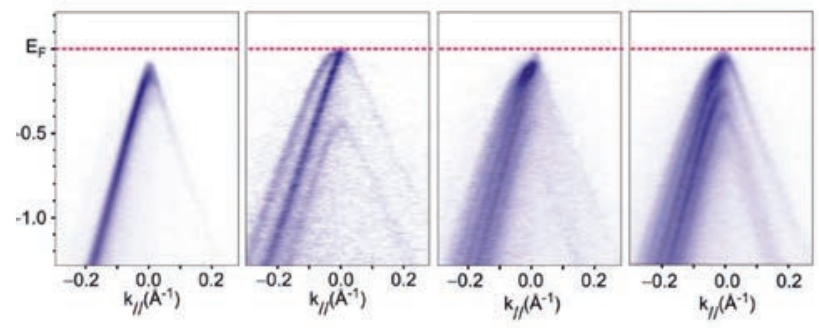

Figure 2.13: (a) LEEM image of FL C-face graphene on $\mathrm{SiC}(000 \overline{1})$ field of view $10 \mu \mathrm{m}$. The area 1 and 2 correspond to $6 \mathrm{ML}$ of graphene. (b) and (c) MicroLEED diffraction patterns collected using a probing area of $400 \mathrm{~nm}$ from the area 1 and 2. respectively. (d) Micro-LEED pastern of collected using a probing area of $5 \mu \mathrm{m}^{2}$, the sampling area includes area 1 and 2. (e) Micro-ARPES of C-face graphene showing a Dirac-like band structure. (f) Nano-ARPES of different Cface graphene domains showing that the band structure is composed of multiple $\pi$-bands. (a), (b), (c) and (d) adapted with permission from Ref. 119. Copyrighted by the American Physical Society. (e) and (f) are reprinted with permission from Ref. 120.

\subsection{Si- and C-face graphene on $3 \mathrm{C}-\mathrm{SiC}(111)$}

Si-face EG grown on 3C-SiC(111) exhibits similar structural properties as Si-face $\mathrm{EG}$ on $4 \mathrm{H}-$ and $6 \mathrm{H}-\mathrm{SiC}$ (0001), i.e. graphene grows on top of a buffer layer (Fig. 2.14(c)) [123-125] and is characterized by an excess of electron doping. Siface $\mathrm{EG}$ and C-face graphene can be grown on 3C-SiC(111) on hexagonal $\mathrm{SiC}(0001)$ substrate. In this case, 3C-SiC(111)/4H-or 6H-SiC(0001) epilayers show an adequate crystalline quality. Si-face EG was grown by low- and high-temperature sublimation techniques, where a high-crystalline quality and at least $\sim 2000 \mu \mathrm{m}^{2}$ large-area of homogenous graphene were achieved (Fig. 2.14(a)) [56, 123, 126]. Yazdi et. al. compared the Si-face EG formation on 4H-SiC(0001), 6H-SiC(0001) and $3 \mathrm{C}-\mathrm{SiC}(111)$ under the same growth conditions using high-temperature sublimation. They found that Si-face EG on 3C-SiC(111) exhibits a better thickness uni- 

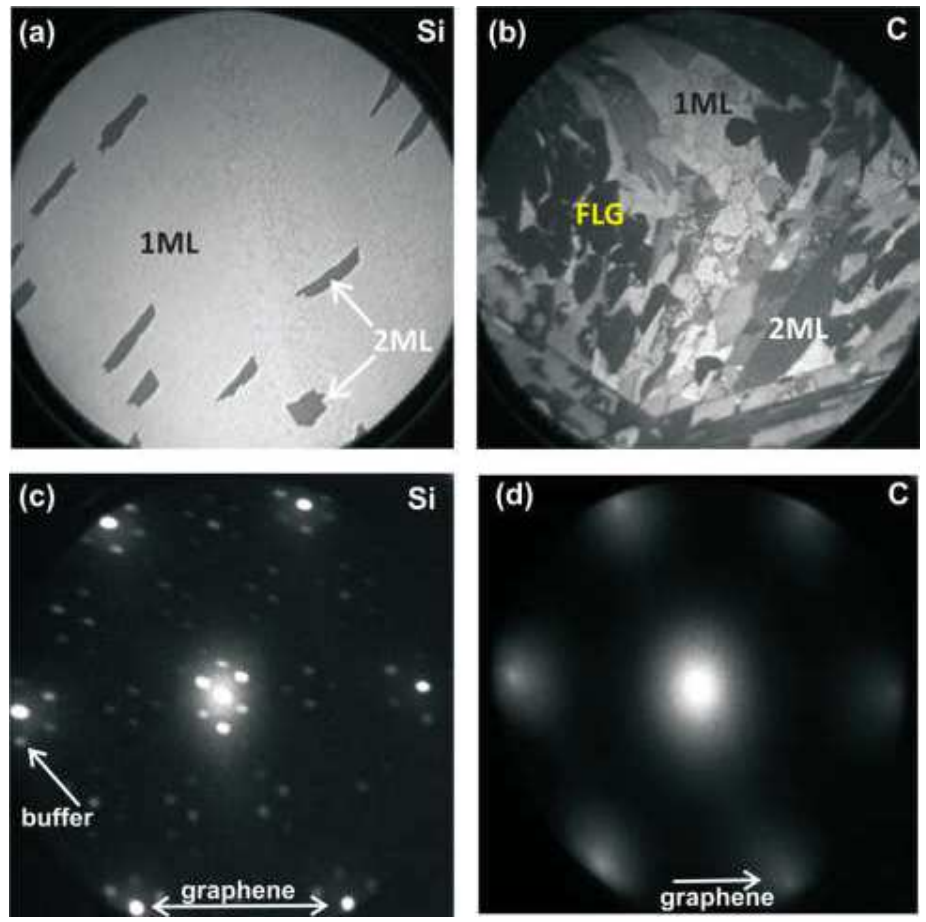

Figure 2.14: LEEM images from selected sample areas for Si-face EG (a) and (b) C-face graphene on 3C-SiC(111)/6H-SiC (field of view $50 \mu \mathrm{m}$ ). Domains with 1, 2 and few layer (FL graphene: 3 and 4 monolayers) graphene are indicated on the LEEM images. Micro-LEED pattern from a monolayer area of the Si-face (c) and C-face (d) taken at 40 and $44 \mathrm{eV}$, respectively. Reprinted with permission from Ref. 123 .

formity compared to $4 \mathrm{H}-\mathrm{SiC}(0001), 6 \mathrm{H}-\mathrm{SiC}(0001)$ over an area of $\sim 2000 \mu \mathrm{m}^{2}$ [56]. Darakcheiva et. al. compared the Si-face EG and C-face graphene formation on 3C-SiC(111)/6H-SiC(0001) grown by high-temperature sublimation in argon [123]. On Si-face EG a large-area of homogenous monolayer graphene up to $\sim 2 \times 2 \mathrm{~mm}^{2}$ can be achieved. However, the C-face graphene consists of small domains with uniform thickness (Fig. 2.14(b)). The micro-LEED investigations showed that C-face graphene in this case forms without a buffer layer (Fig. 2.14(d)). The structural properties, stacking order, interaction between the individual layers, as well as the free charge carrier properties in FL C-face graphene on 3C-SiC(111) are not well understood. Si-face EG can be also grown on 3C-SiC(111) epilayer on Si substrate, 3C-SiC(111)/Si(111). This approach facilitates the integration of EG in conventional Si technology but Si-face EG in this case is non-uniform over large-area and has a low crystalline quality $[125,127,128]$. 
(a)

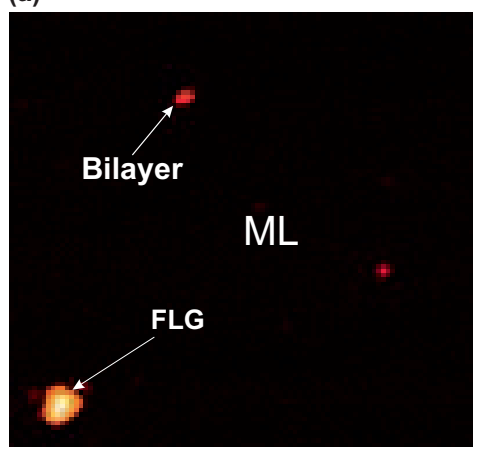

(b)

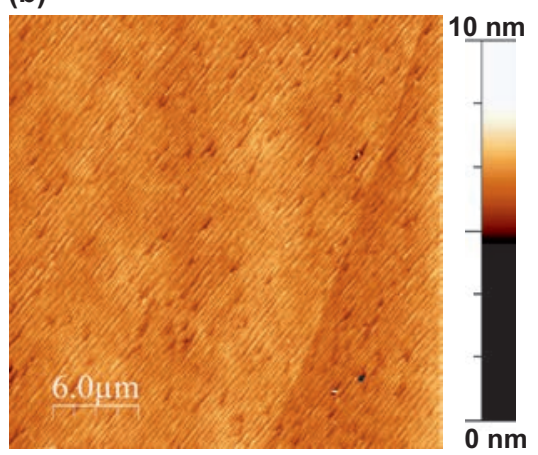

Figure 2.15: (a) $30 \times 30 \mu \mathrm{m}^{2}$ thickness map of Si-face EG on $4 \mathrm{H}-\mathrm{SiC}(0001)$ with $98 \%$ of monolayer graphene coverage. (b) $30 \times 30 \mu \mathrm{m}^{2}$ AFM image of Si-face EG on $4 \mathrm{H}-\mathrm{SiC}(0001)$. Adapted from Paper VII.

\subsection{Samples studied in the thesis}

In this thesis, I have investigated Si-face EG and C-face graphene grown on $4 \mathrm{H}-$ $\mathrm{SiC}$ (0001) and 3C-SiC (111). The graphene samples were grown by high-temperature sublimation in custom build reactors at the sublimation laboratory at IFM. The C-face graphene samples were provided by Prof. Rositsa Yakimova. The Siface EG sample were grown in a newly designed crucible as shown in Fig. 2.12(a). Figures. 2.15(a) and (b) show a typical thickness map and an AFM image, respectively of Si-face EG on $4 \mathrm{H}-\mathrm{SiC}(0001)$ grown in our new crucible (Fig. 2.12(b)). The reflectance map shows uniform monolayer graphene $(98 \%)$ over $900 \mu \mathrm{m}^{2}$. The AFM shows uniform surface morphology with an average terrace step height of $0.5 \mathrm{~nm}$. The $4 \mathrm{H}-\mathrm{SiC}$ substrates used in this thesis are from Cree Inc. The Siface $4 \mathrm{H}-\mathrm{SiC}(0001)$ are chemically mechanically polished (CMP) epiready surfaces with an on-axis orientation. We have used mechanically polished (MP) and CMP polished epiready surfaces on-axis C-face $4 \mathrm{H}-\mathrm{SiC}(000 \overline{1})$ substrates. 3C-SiC(111) substrates are not commercially available. Thick 3C-SiC(111) films were grown by sublimation on $6 \mathrm{H}-\mathrm{SiC}(0001)$ in house within the NetFISiC project. The as-grown 3C-SiC(111) quasi-substrates were CMP processed by NOVASiC. 


\section{Chapter 3}

\section{Experimental techniques}

\subsection{Raman scattering Spectroscopy}

Raman spectroscopy describes the inelastic scattering of light by the Raman active optical phonons of a crystal. Raman scattering consists of the following processes: i) a phonon with frequency $\omega_{i}$ and a wavevector $\vec{k}_{i}$ interacts with a crystal and an optical transition occurs by exciting an electron-hole pair from the valence to the conduction band, ii) the excited electron interacts with Raman-optical active phonon with a frequency $\omega_{0}$ and a wavevector $\vec{q}$ either by gaining (or losing) energy $\hbar \omega_{0}$, therefore becoming in a virtual state, iii) the electron recombines at the valence band by emitting a photon with $\omega_{s}=\omega_{0}+\omega_{i}$ and $\vec{K}_{s}=\vec{q}+\vec{k}_{i}$ (so called Stokes) or with $\omega_{s}=\omega_{0}-\omega_{i}$ and $\vec{K}_{s}=\vec{q}-\vec{k}_{i}$ (so called anti-Stokes). The first-order Raman process corresponds to an optical phonon with $\vec{q} \sim 0$ and the second-order Raman process involves two optical phonons where their energy and momentum are conserved. The difference between emitted photon frequency and the incident light frequency corresponds to the Raman shift. The Raman spectrum of graphene is typically measured in Stokes configuration and exhibits three main Raman peaks: G, D and 2D peaks (Fig. 1.6).

The $G$ peak, located at $1582 \mathrm{~cm}^{-1}$, corresponds to the $E_{2 g}$ vibrational mode and it is a first-order Raman scattering process. The $\mathrm{D}$ and $2 \mathrm{D}$ peaks are associated with $\mathbf{K} / \mathbf{K}^{\prime}$ intervalley double resonance Raman scattering processes and involve two TO phonons. The D mode is Raman inactive and requires a defect to be activated. The D peak is caused by an intravalley double resonant Raman scattering process and it appears for graphene layers with significant amount of defects $[20,129,130]$. The $\mathrm{D}$ and 2D peak positions are blueshifted with increasing the laser energy. The $G\left(\omega_{G 0}\right)$ and $2 D\left(\omega_{2 D 0}\right)$ peak positions for freestanding graphene (undoped and unstained graphene) are $1582 \mathrm{~cm}^{-1}$ and $2670 \mathrm{~cm}^{-1}$, respectively for an excitation wavelength of $532 \mathrm{~nm}$. 


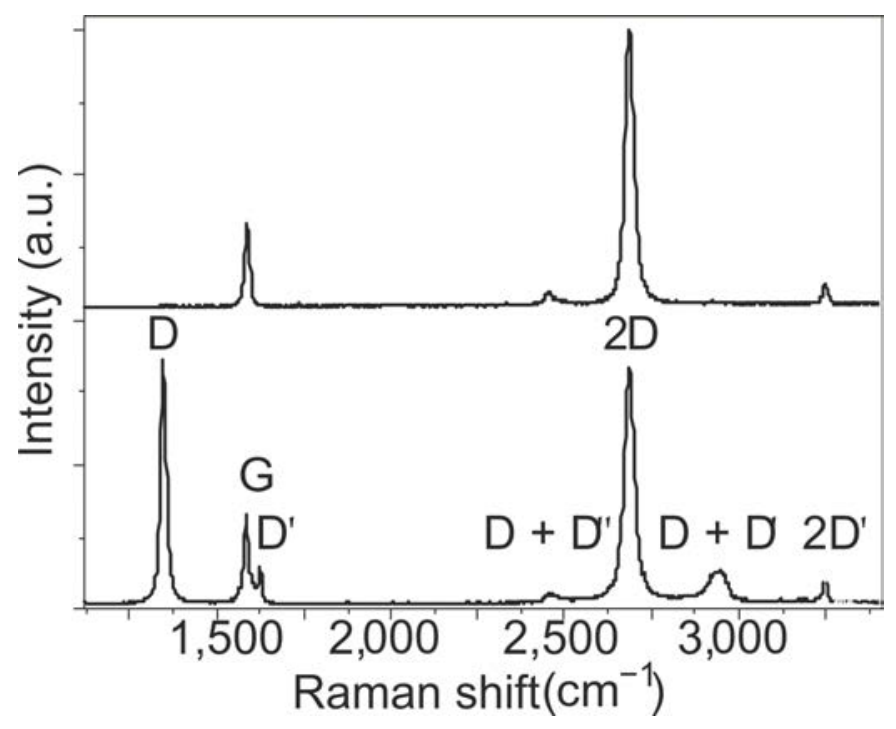

Figure 3.1: Raman spectra of pristine (top) and defected (bottom) graphene, showing the main Raman D, G and 2D peaks. Reprinted with permission from Ref. 130.

\subsubsection{The effects of doping}

Electron and hole doping has a major effect on the the Raman spectra of graphene and it has been investigated on gated exfoliated graphene [131]. Figs. 3.1.1(a) and (b) show the position $\omega_{G}$ and the full width at half maximum (FWHM(G)) of the $\mathrm{G}$ peak as a function of electron and hole doping, respectively. Both electron and hole doping blueshift the $G$ peak position with respect to those of undoped graphene by $\delta \omega_{G}^{e} / \delta E_{F}=30 \mathrm{~cm}^{-1} \mathrm{eV}^{-1}$ and $\delta \omega_{G}^{h} / \delta E_{F}=42 \mathrm{~cm}^{-1} \mathrm{eV}^{-1}$ for electron and hole doping, respectively [131, 132]. The FWHM(G) decreases for both electron and hole doping and saturates for a Fermi energy higher than half of the photon energy. The 2D peak position and FWHM(2D) exhibit different behaviors compared to the $G$ peak. The $\omega_{2 D}$ shows a quasi-linear shift to higher frequency for hole doping by $\delta \omega_{2 D}^{h} / \delta E_{F}=32 \mathrm{~cm}^{-1} \mathrm{eV}^{-1}$, while for electron doping, the $2 \mathrm{D}$ position first shifts to higher energies for concentrations up to $2-3 \times 10^{-13} \mathrm{~cm}^{-2}$, then drastically decreases [131, 133], as shown in Fig. 3.1.1(c). The FWHM(2D) increases with increasing the electron and hole concentrations. The increase of the FWHM(2D) with increasing doping was attributed to carrier interactions [134, 135]. The increases in doping ads to decrease of the ratio between $2 D$ and $G$ peak intensities, $I(2 D) / I(G)$, and their integrated intensities, $A(2 D) / A(G)$ [136] (Fig. 3.1.1(d)). These variations of Raman peak characteristics can be used to estimate the doping of graphene. 
(a)

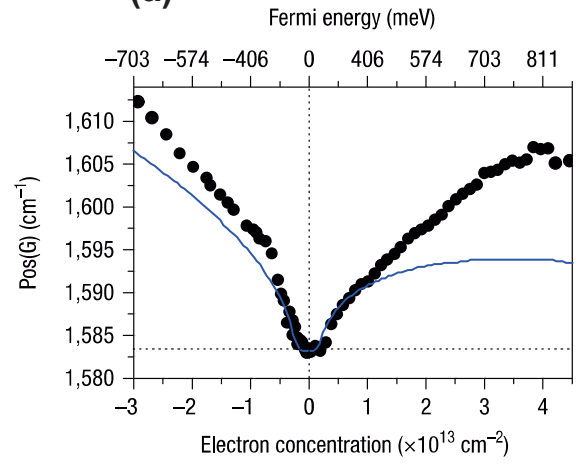

(c)

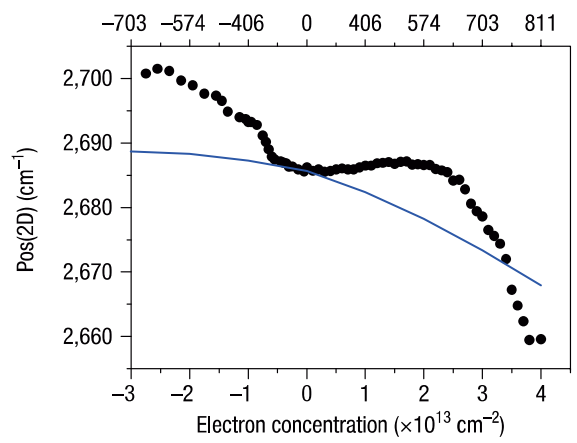

(b)

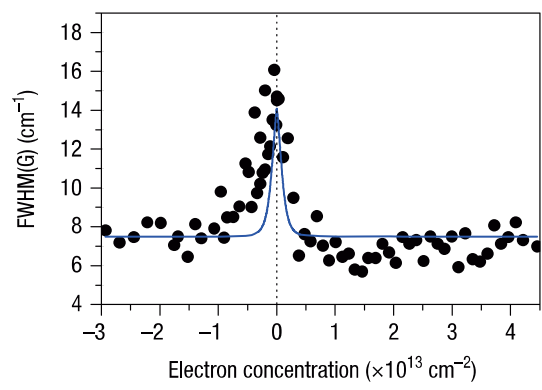

(d)

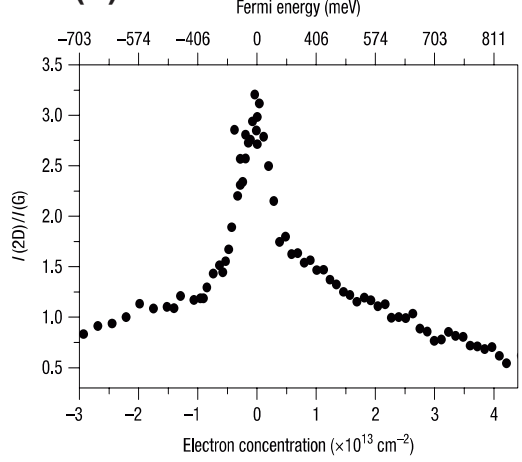

Figure 3.2: (a) G peak position, (b) full width at half maximum of G peak, (c) 2D peak position and (d) ratio between $2 D$ and $G$ peak intensities, as a function of hole and electron doping in gated exfoliated graphene. Adapted from Ref. 131.

\subsubsection{The effects of strain}

Strain also can induce major changes in the Raman spectra of graphene. Tensil biaxial strain $(\varepsilon)$ redshifts the $G$ and $2 D$ peak positions with respect to $\omega_{G 0}, \omega_{2 D 0}$. The $G(2 D)$ peak position shift due to biaxial strain is described by [137]

$$
\Delta \omega_{G(2 D)}^{b}=-2 \omega_{G(2 D), 0} \gamma_{G(2 D)^{\mathcal{E}}}
$$

where $\gamma_{G(2 D)}$ is the Grüneisen parameter of the $G(2 D)$ peak. Uniaxial strain shifts the $G$ and $2 D$ positions to lower frequencies with respect to $\omega_{G 0}, \omega_{2 D 0}$. Uniaxial strain applied along either zigzag or armchair directions changes the crystal symmetry of graphene. In such case, distorted graphene belongs to the $D_{2 h}$ point 
group and consequently, the $E_{2 g}$ degeneracy is lifted off and the $G$ peak splits into $\mathrm{G}^{+}$and $\mathrm{G}^{-}$peaks. In order to determine the direction, along which strain is applied, polarized Raman measurements are typically employed [137, 138].

\subsubsection{The effects of number of layers and stacking}

Ferrari et. al. showed that the number of graphene layers and their stacking can be determined for exfoliated graphene from the shape and FWHM of the 2D peak [129]. Fig. 3.1.4(a) shows the Raman spectra for exfoliated graphene in the vicinity of the $2 \mathrm{D}$ peak as a function of the number of graphene layers. Monolayer graphene has a symmetric $2 \mathrm{D}$ peak, which can be fitted using one Lorentzian peak, with a $\mathrm{FWHM}(2 \mathrm{D}) \approx 25 \mathrm{~cm}^{-1}$. However, $A B$-stacked bilayer graphene shows a broad and asymmetric peak, with a $\operatorname{FWHM}(2 \mathrm{D}) \approx 55 \mathrm{~cm}^{-1}$, which can be fitted using four Lorentzian peaks reflecting the parabolic band structure of bilayer graphene [20, 129].

\subsubsection{Raman Spectroscopy of graphene on $\mathrm{SiC}$}

Si-face and C-face EG on $\mathrm{SiC}$ are doped and strained, consequently their $\omega_{G}$ and $\omega_{2 D}$ are typically shifted from the strain-free position of undoped graphene $\left(\omega_{G 0}\right.$, $\omega_{2 D 0}$ ) [139]. Since these shifts are a combination of the effects caused by the strain and doping, the determination of doping type for EG is not feasible from Raman spectroscopy alone. If the doping type is known, the carrier concentration and strain can be estimated by solving a system of two equations with two unknown parameters [140]. In contrast to the case of exfoliated graphene, the determination of the number of layers in Si-face EG and C-face graphene by Raman spectroscopy is not a trivial task. Strain nonuniformity, defects, doping, and stacking order, are several factors that may change the shape of the 2D peak and the FWHM(2D) in EG [141, 142]. The analysis of the 2D peak shape can be used to investigate the interaction between the individual layers in the FL C-face graphene stack. For example, Fig. 3.1.4 shows the Raman spectra of monolayer graphene and FL $\mathrm{C}$-face graphene on $4 \mathrm{H}-\mathrm{SiC}(000 \overline{1})$ grown using the crucible shown in Fig. 2.12. The monolayer and FL C-face graphene show a symmetric Lorentzian peak with FWHM(2D) of about 34 and $24 \mathrm{~cm}^{-1}$, respectively and indicate that the FL C-face graphene behave as a single layer graphene.

In this thesis, we have used three different Raman systems: Raman setup at IFM (see section 3.2), Raman setup at Laboratoire Charles Coulomb (University of Montpellier, France), and Renishaw system at the University of Nebraska-Lincoln (U.S.A.). 
(a)

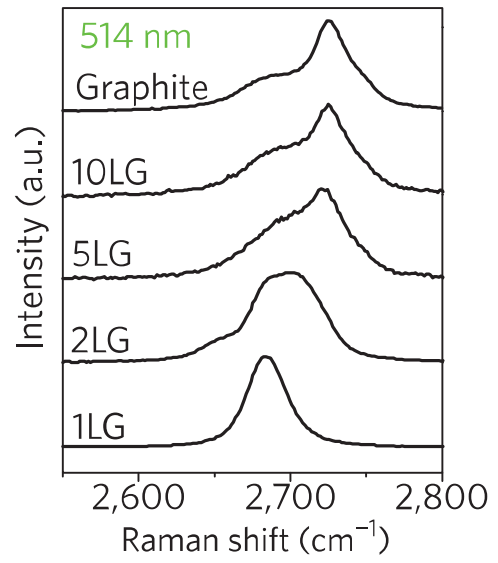

(b)

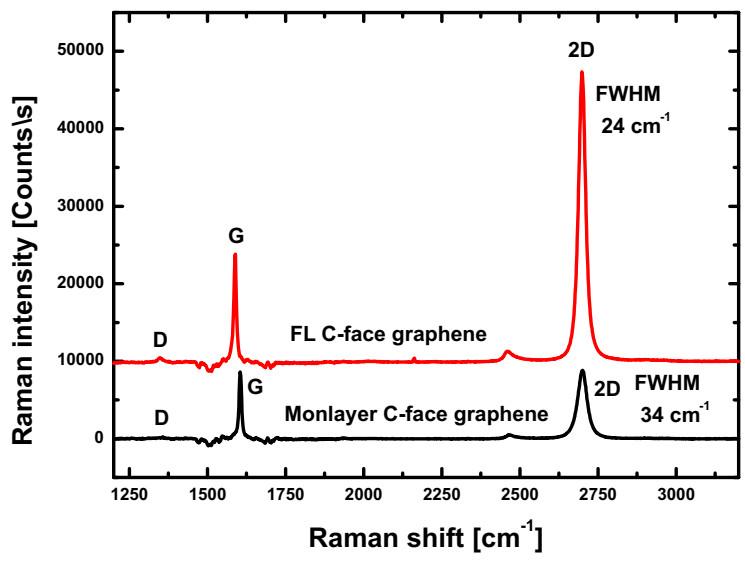

Figure 3.3: (a) Raman spectra in the vicinity of the 2D peak for different numbers of graphene layers. Reprinted with permission from Ref. 130. The Raman spectra are obtained from exfoliated graphene. (b) Raman spectra of monolayer (black

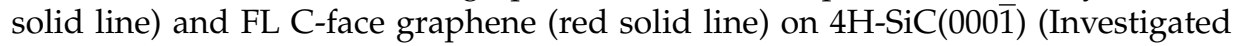
in the thesis).

\subsection{Reflectance}

Reflectance can be used to determine the number of graphene layers and map their distribution over the sample surface. Upon reflection, the intensity of light is changed as a result of interaction with graphene. Thus, the intensity of the reflected light from the graphene sample $R(N)$ can be used to determine the number $\mathrm{N}$ of graphene layers using [143]

$$
R(N)=R_{S i C}(1+0.017 N),
$$

where $R_{S i C}$ is the reflectance of a bare $\mathrm{SiC}$ reference. The intensity of the reflected light increases linearly by $1.7 \%$ per graphene layer for up to 12 layers.

Fig. 3.4 shows the reflectance experimental setup used in this thesis. The setup is based on a similar optical configuration as a conventional micro-Raman system, where two beam splitters (BS1 and BS2) were added. BS1 splits the incident laser beam and directs the laser beam after reflection from the sample to BS2. The role of BS2 beam splitter is to divide the laser beam into two beams: one reflected the CCD camera used as a feedback loop to control the focus on the sample via a piezoelectric system, while the other is reflected on the power meter. The samples are mounted on a three-axis piezoelectric stage used for scanning the sample dur- 


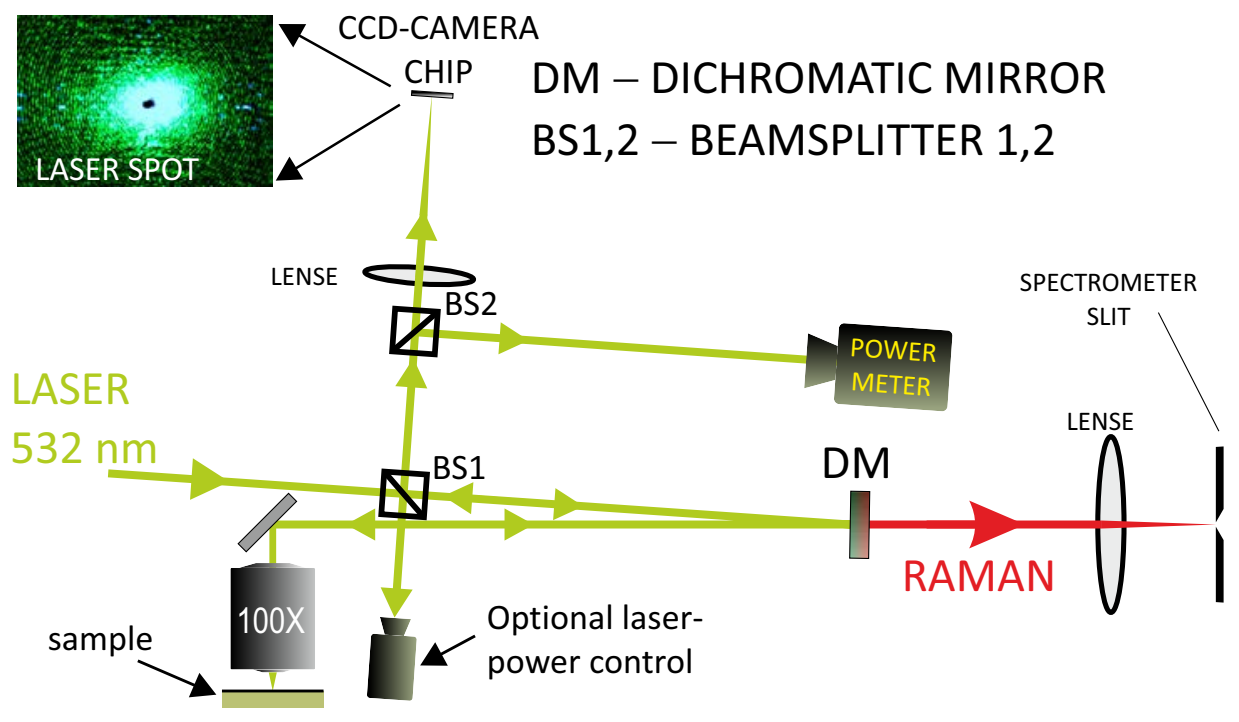

Figure 3.4: Schematic representation of the reflectance setup at LiU. Adapted from Ref. 143.

ing the acquisition, to perform the reflectance mapping and focusing of the laser spot. The focus of the laser beam over the sample is achieved via a $100 \times$ objective. The FWHM of the laser spot is about $400 \mathrm{~nm}$. A diode-pumped semiconductor laser with a wavelength of $532 \mathrm{~nm}$ (photon energy $\mathrm{E}_{L}=2.33 \mathrm{eV}$ ) was used as an excitation source [143].

This system has the capability to perform reflectance and micro-Raman mapping simultaneously. This allows to assign a specific Raman spectrum to the respective number of layers and draw a conclusion about the properties of graphene, such as coupling/decoupling, doping, and strain in relation to the number of layers.

\subsection{Scanning probe microscopy techniques}

\subsubsection{Atomic force microscopy}

Atomic force microscopy (AFM) was used in this thesis to measure the morphology (topography) of the graphene samples. The AFM uses a cantilever equipped with a sharp tip. The tip interacts with the sample surface via different forces, such as: Van de Waals force, mechanical contact, etc, which cause the deflection or oscillation of the tip. A detection system including laser, photodetector and 
piezoelectric crystal is used to detect and measure this interaction during a scan over the sample. The AFM measurements are typically performed in the contact, non-contact or tapping modes. In contact mode operation, the AFM tip is in a mechanical contact with the sample surface inducing a deflection of the cantilever due to the repulsive forces between the tip and the sample. Such deflection is used as a feedback signal to measure the surface topography [144]. The tapping-AFM mode (dynamic scan mode) is used to measure the surface topography of soft samples, such as graphene, to avoid the surface damage that can be caused by using the contact mode. In the tapping mode, the cantilever oscillates with a frequency close to the resonance frequency of the piezoelectric crystal, which depends on the tip and cantilever properties. During the surface scan, the oscillating tip taps the surface of the sample inducing a change of its oscillation amplitude. These changes are used to measure the surface topography. A feedback loop keeps the oscillation amplitude constant by adjusting the distance tip-sample in order to ensure constant tip-surface interaction during the scanning [144, 145]. In this thesis, the graphene surface morphology was investigated using the AFM tapping mode and with a Veeco DI3100 AFM equipment at IFM.

\subsubsection{Conductive atomic force microscopy}

Conductive atomic force microscopy (CAFM) allows simultaneous measurements of surface topography and the local electrical conductivity map of a surface. The conventional CAFM uses scanning contact-AFM mode to measure surface morphology, in which the local current maps are obtained by applying a DC bias between a conductive tip in contact mode and the sample backside. A low-noise linear current amplifier senses the resulting electrical current through the sample, as illustrated in Fig. 3.5(a). Apart from conductivity maps, the CAFM can also be used to measure local current-voltage $\left(I-V_{\text {tip }}\right)$ curves at a specific position of the topographic or current map. However, the CAFM is not suitable for investigating graphene sample due to the high shear forces that can damage the graphene sheets. Instead, torsion resonance conductive atomic force microscopy (TR-CAFM) was used in this thesis to investigate the local electrical conductivity of graphene samples. TR-CAFM is based on the same principle as CAFM, but the tip is oscillating in a torsional or twisting mode in close proximity to the sample surface $(0.3-3 \mathrm{~nm})[146,147]$. Consequently, the current flows from the tip to the graphene sample backside via tunneling when a DC bias is applied. Fig. 3.5 (a) and (b) show typical topographic and current conductivity maps of FL C-face graphene on $4 \mathrm{H}-\mathrm{SiC}(000 \overline{1})$, respectively. 
(a)

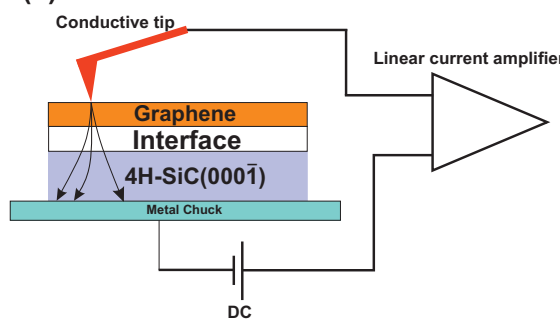

(b)

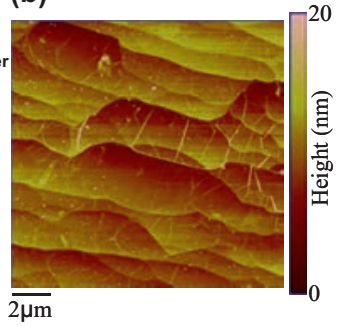

(c)

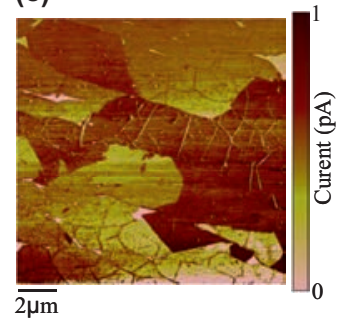

Figure 3.5: (a) Schematic representation of torsion resonance conductive atomic force microscopy. (b) TR-CAFM topography and (b) the corresponding current map of FL C-face graphene on $4 \mathrm{H}-\mathrm{SiC}(000 \overline{1})$. Adapted from Paper II.

In this thesis, TR-CAFM measurements were carried out using a Veeco DI3100 AFM with Nanoscope $\mathrm{V}$ controller and tunneling atomic force microscopy (TUNA®) application module. Platinum-coated $\mathrm{Si}$ tips with a typical apex radius of $10-20 \mathrm{~nm}$ were used. From selected positions of the current maps, local $I-V_{\text {tip }}$ measurements were carried out by scanning current spectroscopy where the conductive tip was in direct contact with graphene. The measurement were performed at CNR-Institute for Microelectronics and Microsystems (Italy).

\subsubsection{Scanning Kelvin probe microscopy}

Scanning Kelvin probe microscopy (SKPM) allows to simultaneously measure the surface topography and to probe the local contact potential difference (CPD) between a conducting tip and a sample. When the tip and sample are not connected, their vacuum level are aligned but their Fermi energies are different (Fig. 3.6(a)). However, when the tip is in close proximity to the sample surface, the work function difference between the tip $\left(\phi_{\text {tip }}\right)$ and the sample surface $\left(\phi_{\text {sample }}\right)$ cause a tunneling electron current flow. In this case, the Fermi energy of the tip and sample surface line-up and the system reaches an equilibrium state. Therefore, the sample and tip are charged, and have now different vacuum levels. As a result of charging of the tip and the sample, an electrostatic force is created and an apparent $V_{\mathrm{CPD}}$ is formed between the tip and sample surface [144], as illustrated in Fig. 3.6(b). $V_{\mathrm{CPD}}$ can be compensated when an external bias $V_{\mathrm{DC}}$ with the same magnitude and opposite sign to $V_{\mathrm{CPD}}$ is applied to the tip, and consequently the force between the tip and the sample will be canceled as seen in Fig. 3.6(c). In this thesis, we have used amplitude modulated SKPM, which is a two-pass technique: i) during the first pass, the surface topography is measured using tapping-AFM mode, ii) the second pass uses the saved surface topography to maintain the tip 
(a)

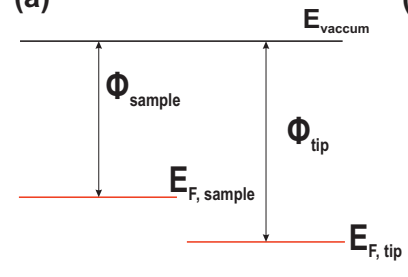

(b)

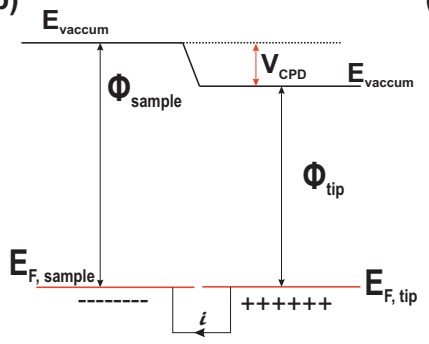

(c)

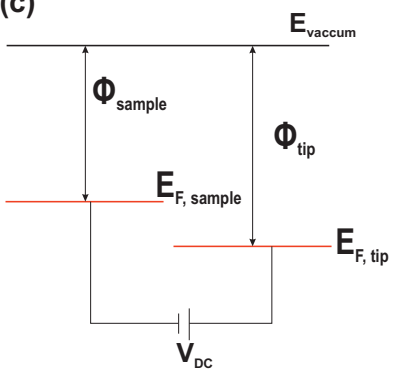

Figure 3.6: Schematic diagram of the energy levels of: (a) the AFM-tip and the sample surface in noncontact mode, (b) the AFM-tip and the sample close in the proximity, (c) the AFM tip and the sample in close proximity when $V_{\mathrm{DC}}$ bias is applied to nullify $V_{\mathrm{CPD}}$ and consequently the electrostatic forces. $\mathrm{E}_{\mathrm{vacuum}}$ denotes the vacuum level [144].

at certain constant lift height of $10-20 \mathrm{~nm}$ and the tip oscillation caused by the piezoelectric crystal is disabled. During the second pass an $A C\left(V_{A C}\right)$ and $D C$ $\left(V_{\mathrm{DC}}\right)$ biases are applied to the tip. The frequency of the $\mathrm{AC}$ bias is similar to the resonance frequency of the tip-cantilever $(\omega)$. The electrostatic forces between the non-contacting tip and the sample surface are very small [148]. The $V_{A C}$ is used to increase the sensitivity while the $V_{\mathrm{DC}}$ is used to nullify the tip-sample forces. The electrostatic forces between tip-sample can be expressed as [144]

$$
F(z, t)=-\frac{1}{2} C(\Delta V)^{2}
$$

where

$$
V=V_{C P D}-V_{D C}+V_{A C} \sin (\omega t) .
$$

Inserting Eqn. 3.4 into the Eqn. 3.3 gives

$$
\begin{aligned}
F(z, t)= & -\frac{1}{2} \frac{d C}{d z}\left(V_{C P D}-V_{D C}\right)^{2} \\
& -\frac{d C}{d z}\left(V_{C P D}-V_{D C}\right) \times V_{A C} \sin (\omega t) \\
& \left.+\frac{1}{4} \frac{d C}{d z}\left(V_{A C}^{2} \cos (2 \omega t)-1\right)\right) .
\end{aligned}
$$

The $V_{\mathrm{CPD}}$ value can be measured by using a feedback loop that nullifies $\mathrm{F}(\mathrm{z}, \mathrm{t})$ by adjusting $V_{\mathrm{DC}}$. The local work function of graphene $W_{G}$ is related to $V_{\mathrm{CPD}}$ by

$$
W_{G}=\phi_{\text {tip }}-e V_{C P D}
$$




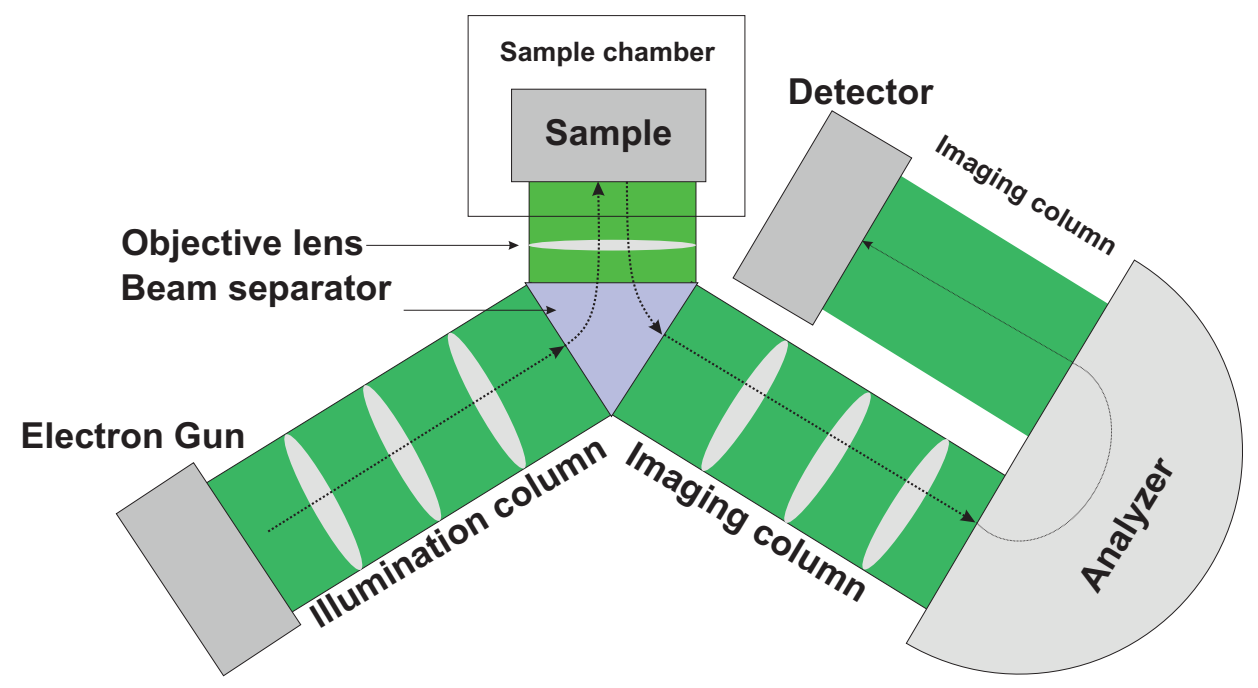

Figure 3.7: Schematic representation of the ELMITEC-LEEM III instrument. Adapted from Ref. 149 .

where $\phi_{\text {tip }}$ and $e$ are the work function of the tip and the elementary charge, respectively. The absolute local work function of graphene is difficult to quantify due the change of $\phi_{\text {tip }}$ during the SKPM scans caused by attachment of adsorbates present at graphene surface and the tip, variation of ambient humidity, tip deformation etc. However, one can investigate the local variations of the work function which can be expressed independently of $\phi_{\text {tip }}$.

In this thesis, SKPM was performed at room temperature and ambient conditions using a Veeco DI 3100 scanning probe microscope equipped with Nanoscope IV controller. Conductive Pt/Ir-coated Si tips (Nanosensors PPP-EFM-20) with resonance frequencies between 45 and $115 \mathrm{kHz}$ was used [148].

\subsection{Surface techniques}

\subsubsection{Low-energy electron microscopy}

Low-energy-electron microscopy (LEEM) uses low-energy elastically backscattered electrons for surface imaging. Fig. 3.7 shows a schematic illustration of the used LEEM instrument with a resolution $\sim 5 \mathrm{~nm}$ used in this thesis. In LEEM measurements, the electrons are emitted by an electron gun with high energy and guided to the sample via the illumination column. A beam separator bends the electrons to the sample through an objective lens. As they approach the sample, the electrons are decelerated by a negative potential held between the electron gun 
(a)

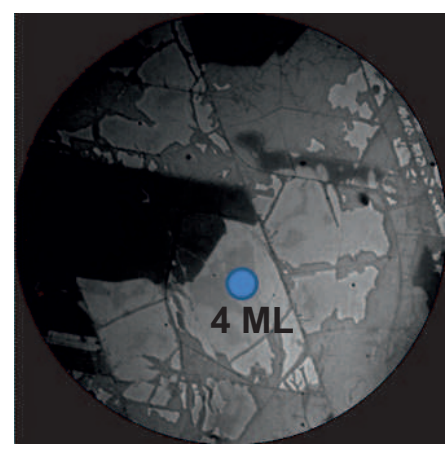

(b)

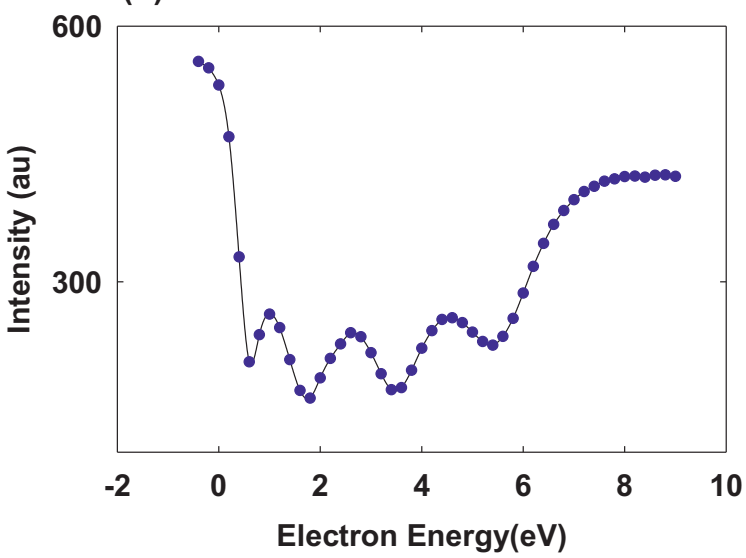

Figure 3.8: (a) LEEM image of FL C-face graphene on 3C-SiC(111) acquired at an electron energy of $2.8 \mathrm{eV}$ and with a field of view of $30 \mu \mathrm{m}$, (b) electron reflectivity $I-V$ spectrum extracted from the area indicated by a circle in (a). Adapted from Paper III.

and the sample, reaching a low energy in the range of $0-100 \mathrm{eV}$. The energy of the incident electron can be adjusted by changing the potential (bias voltage) between the gun and the sample. The backscattered electrons are then reaccelerated through the same objective lens and deflected by a beam separator to the imaging column. The objective lenses and the imaging column induce a magnification of the probed area. The LEEM instrument can be used also in a diffraction mode. In this case, the elastically backscattered electrons form low energy diffraction patterns of the sample surface at the backfocal plane of the objective lens. If an illumination aperture is introduced to the objective lens, a selected area-low energy diffraction (micro-LEED) can be performed on the sample [150, 151].

The surface morphology and the crystallographic structure of the sample surface induce a phase contrast in the LEEM image. Electron reflectivity curves (I-V) are used to extract the number of graphene layers. Fig 3.8(a) presents a LEEM image of FL C-face graphene on $3 \mathrm{C}-\mathrm{SiC}(111)$, and the corresponding electron reflectivity spectrum from selected area indicted by the circle in Fig 3.8(b). The electron reflectivity spectra are characterized by the presence of minima that are due to the constructive and destructive interference caused by the electron waves reflected from the different graphene layers [152]. The number of graphene layers can be determined by counting the number of minima in the reflectivity spectrum, e.g., the area indicated by a circle in Fig 3.8(b) exhibits an $I-V$ curve with four minima which correspond to four graphene layers. 
(a)

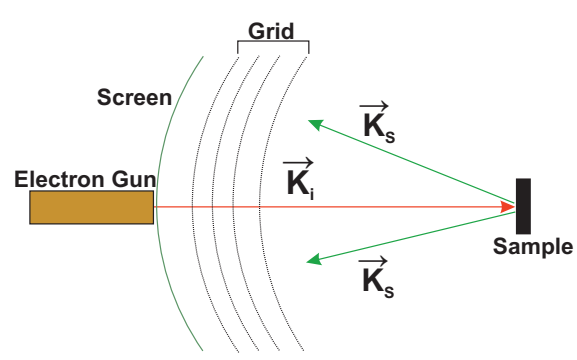

(b)

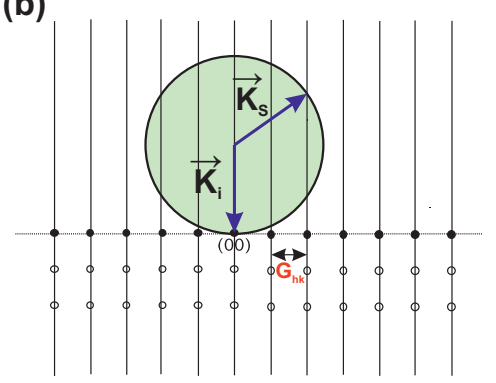

Figure 3.9: (a) Schematic representation of a LEED experiment. (b) Ewald sphere construction in two dimensions.

In this thesis, LEEM measurements were performed in ultra-high vacuum (UVH) conditions, in a ELMITEC-LEEM III instrument at the I311 beamline of the MAX-Lab synchrotron radiation facility in Lund (Sweden).

\subsubsection{Low-energy electron diffraction}

Low-energy electron diffraction (LEED) is a surface technique that can determine the crystallographic structure of a surface. In a LEED experiment, a low energy electron beam, with a wavevector $\vec{k}_{i}=2 \pi / \lambda_{i}$, is directed to a sample surface at normal incidence, then the electrons are backscattered: $i$ ) elastically with a wavevector $\vec{k}_{s}=2 \pi / \lambda_{s}$ and ii) inelastically (filtered out using energy-filtering grids). The elastically scattered electrons are then accelerated by a potential between the grids and a fluorescent screen. A schematic of a LEED experiment is shown in Fig. 3.9(a). In order to observe a diffraction (interference) pattern, the incident and the reflected beams must obey Laue equation

$$
\vec{k}_{0}-\vec{k}_{1}=\vec{G}_{h k},
$$

where $\vec{G}_{h k}$ is the lattice vector of the sample surface. Since we only consider the elastically scattered electrons, then, $\left|\vec{K}_{0}\right|=\left|\vec{K}_{1}\right|$. The Ewald sphere is usually used to visualize the Laue equation (Fig 3.9(b)). This sphere is centered at the origin of the incident vector and represents all the possible vectors having the same magnitude and origin as $\vec{k}_{i}$. The intersections between the Ewald sphere with the reciprocal lattice rods indicate the different $\vec{k}_{s}$ vectors that satisfy the Laue equation and the direction of the diffraction spots [153, 154], as sketched in Fig. 3.9. The LEED experiments in this thesis were performed with the LEEM equipment introduced in section 3.4.1. 


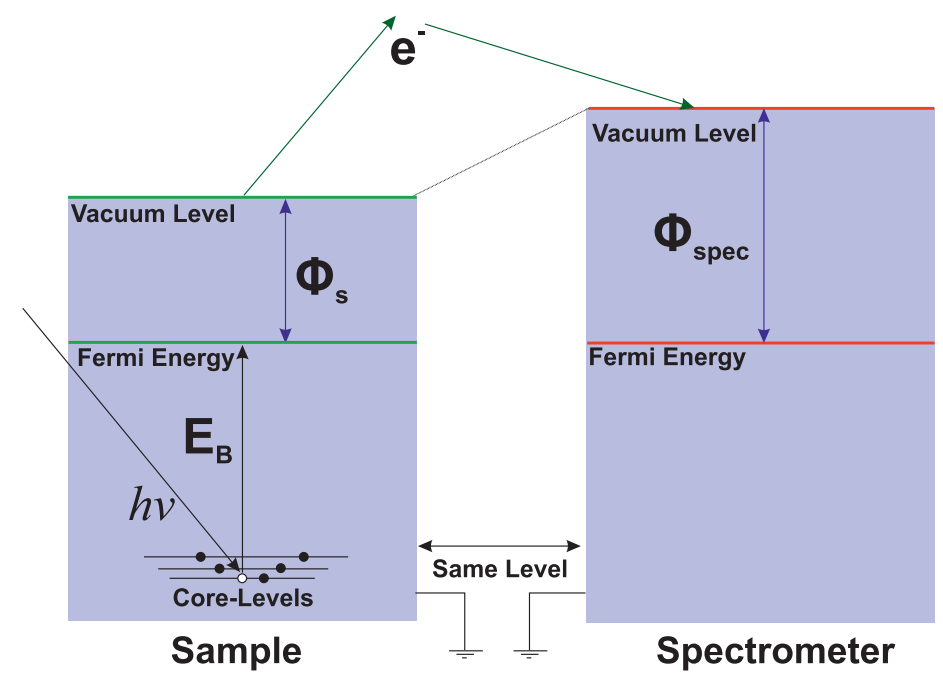

Figure 3.10: Schematic presentation of the XPS emission process.

\subsubsection{X-ray photoelectron spectroscopy and microscopy}

X-ray photoelectron spectroscopy (XPS) is a surface technique used to probe the chemical composition of a sample surface. XPS is based on the photoelectric effect. When a soft-X-ray photon with an energy $(E=\hbar v)$ excites the sample surface, an interaction occurs with an electron (with bonding energy $E_{B}$ ) located in the corelevels of an atom [155]. If the photon energy is higher than $E_{B}$, the energy of the photon is transferred to the electron, which leaves the sample surface with a certain kinetic energy

$$
E_{\mathrm{kin}}=h v-E_{B}-\phi_{s},
$$

where $\phi_{s}$ is the work function of the sample surface. The kinetic energies of the photoexcited electrons are recorded using a spectrometer. The sample and the spectrometer are electrically connected and as a result their Fermi energies are aligned, while their vacuum levels are different (see Fig. 3.10). Consequently, the kinetic energy of the electrons measured at the spectrometer $\left(E_{k i n 1}\right)$ is different and can be expressed as

$$
E_{\mathrm{kin} 1}=E_{\mathrm{kin}}-\left(\phi_{\mathrm{s}}-\phi_{\mathrm{spec}}\right)
$$

where $\phi_{\text {spec }}$ is the work function of the spectrometer. Replacing Eqn. 3.9 in Eqn. 3.9 gives

$$
E_{B}=h v-E_{\mathrm{kin} 1}-\phi_{\mathrm{spec}} .
$$


The bonding energy can be measured if the energy of the photon and the work function of the spectrometer are known parameters. X-ray photoemission electron microscopy (XPEEM) can be used in several modes [156-158]: secondary electron XPEEM, selected area XPS (micro-XPS) and energy filtered XPEEM. Micro-XPS is based on XPS in microscopy mode, performed on a selected area on sample surface (or an area chosen from LEEM image). Energy filtered XPEEM is an imaging mode that uses the electrons emitted from the atomic core levels (XPS), where an energy filter is used to select certain $E_{K i n}$. The image obtained from XPEEM provides information about the lateral chemical composition of sample surface. The XPS and XPEEM measurements reported in this thesis are performed using the synchrotron radiation facility at MAX IV.

\subsection{Spectroscopic ellipsometry}

Spectroscopic ellipsometry (SE) measures the relative change of the polarization state of an incident beam upon reflection (or transmission) from a sample. The incident electromagnetic wave is polarized perpendicular $(s)$ or parallel $(p)$ to the plane of incidence (see Fig. 3.11). The wavevector $k_{i n}$ of the incoming electromagnetic plane wave and the sample normal define the angle of incidence $\phi_{a}$ and the plane of incidence. More precisely, standard SE measures two complex reflectance ratios defined in terms of the ellipsometric angles $\psi$ and $\Delta . \tan \psi$ and $\Delta$ represent the amplitude of the complex ratio and the relative phase changes of the $s$ and $p$ components of the electric field vectors, respectively.

\subsubsection{Types of ellipsometry}

\subsubsection{Standard spectroscopic ellipsometry}

Standard SE is used for optical isotropic materials, where the ellipsometric angles $\psi$ and $\Delta$ do not depend on the polarization state of the incident incoming electromagnetic plane waves, in this case $\psi$ and $\Delta$ are defined by

$$
\rho=\frac{r_{p}}{r_{s}}=\tan \psi \exp (i \Delta)
$$

where $r_{p}$ and $r_{s}$ represent the $p$ - and s-polarized complex reflection coefficients.

\subsubsection{Generalized spectroscopic ellipsometry}

Generalized spectroscopic ellipsometry (GSE) is used for optical anisotropic materials, e.g. $\mathrm{SiC}$, in which the conversion of $p$ - into $s$-polarized light or vice versa 


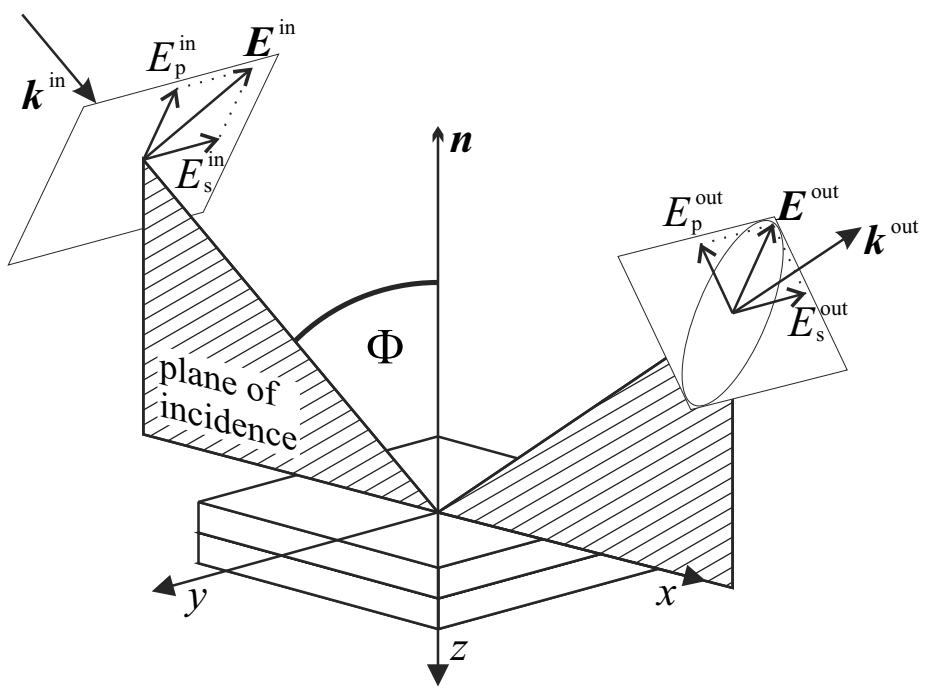

Figure 3.11: Schematic representation of a SE measurement in reflection mode: the amplitudes of the electric field of the incoming $E_{i n}$ and the reflected $E_{\text {out }}$ plane wave can be decomposed into complex field amplitudes $E_{p}^{i n}, E_{s}^{i n}, E_{p}^{o u t}$ and $E_{s}^{\text {out }}$.

occurs. The GSE parameters can be described by using the Jones reflection matrix $r$ or the Mueller matrix formalism.

The Mueller matrix formalism describes the polarization state of the electromagnetic plane waves in terms of intensities. The Stokes parameters of the electromagnetic plane waves are described via the Mueller matrix, before and after sample interaction. In the $s$ - and $p$-coordinate system, the individual Stokes parameters components can be defined by $S_{1}=I_{p}+I_{s}, S_{2}=I_{p}+I_{s}, S_{3}=I_{+45}-I_{-45}$, and $S_{4}=I_{\sigma_{+}}+I_{\sigma_{-}}$, where $I_{p}, I_{s}, I_{+45}, I_{-45}, I_{\sigma_{+}}$, and $I_{\sigma_{-}}$being the intensities for the $p-, s^{-},+45^{\circ},-45^{\circ}$, right- and left-handed circularly polarized light components, respectively. The Mueller matrix $\mathrm{M}$ is the transformation matrix for Stokes vectors [159-161]

$$
\mathrm{S}^{\text {out }}=\mathrm{MS}^{\mathrm{in}},
$$

where $\mathbf{S}^{\text {out }}$ and $\mathbf{S}^{\text {in }}$ denote the Stokes vectors of the electromagnetic plane wave before and after the transformation. The Mueller matrix is a dimensionless, realvalued $4 \times 4$ matrix

$$
\mathbf{M}=\left(\begin{array}{llll}
M_{11} & M_{12} & M_{13} & M_{14} \\
M_{21} & M_{22} & M_{23} & M_{24} \\
M_{31} & M_{32} & M_{33} & M_{34} \\
M_{41} & M_{42} & M_{43} & M_{44}
\end{array}\right) .
$$




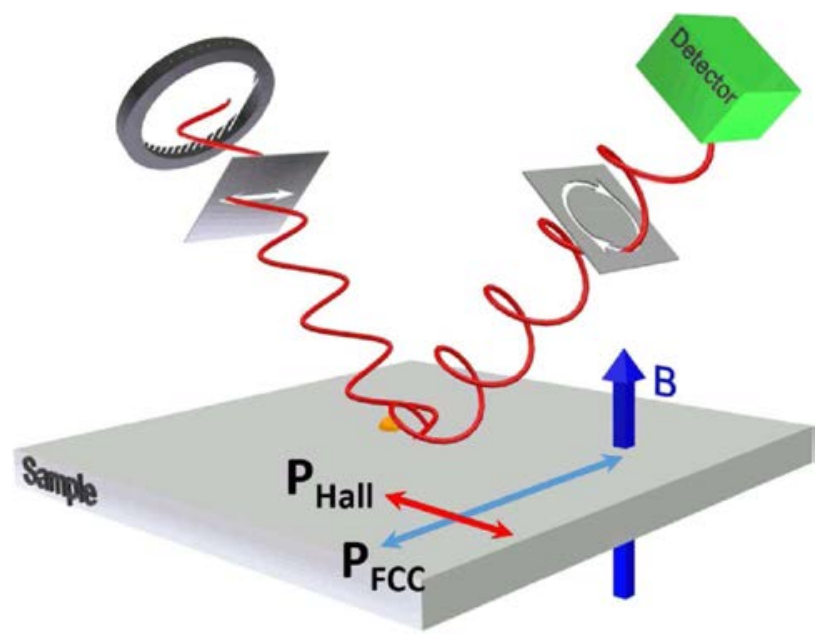

Figure 3.12: Optical Hall effect schematic representation: Free charge carriers induce a dielectric polarization following the electric field of an incident electromagnetic field, here for example parallel to the surface. The induced polarization $\mathbf{P}_{\text {FCC }}$ produces $\mathbf{P}_{\text {Hall }}$ due to the Lorentz force, oriented perpendicular to $\mathbf{B}$ and the incident electric field vector. $\mathbf{P}_{\mathrm{FCC}}+\mathbf{P}_{\text {Hall }}$ are the source of the reflected light and contain a small circular polarization component, which provides information on the type of charge carrier, its density, mobility, and effective mass properties. Reprinted with permission from Ref. 164.

The Muller matrix elements can be decomposed in 4 sub-matrices, where the matrix elements of the two off-diagonal-blocks $\left[\begin{array}{ll}M_{13} & M_{14} \\ M_{23} & M_{24}\end{array}\right]$ and $\left[\begin{array}{ll}M_{31} & M_{32} \\ M_{41} & M_{42}\end{array}\right]$ are nonzero only if $p$-s-polarization mode-conversion appears. The matrix elements in the two on-diagonal-blocks $\left[\begin{array}{ll}M_{11} & M_{12} \\ M_{21} & M_{22}\end{array}\right]$ and $\left[\begin{array}{ll}M_{33} & M_{34} \\ M_{43} & M_{44}\end{array}\right]$ are typically non-zero and contain information about $p$-s-polarization mode-conserving processes [159].

\subsubsection{Optical Hall effect}

\subsubsection{The Optical Hall effect}

The optical Hall effect is the magnetic field-induced birefrengence in the free charge carrier response when interacting with an electromagnetic wave (see Fig. 3.12) [162, 163]. When a sample is excited with an incident electromagnetic plane wave with an angular frequency $(\omega)$ and a linear polarization parallel to the sample, the free charge carriers oscillate in a straight line in the direction of the time-varying electric field ( $\left.\mathbf{P}_{\mathrm{FCC}}\right)$ (see Fig. 3.12). 


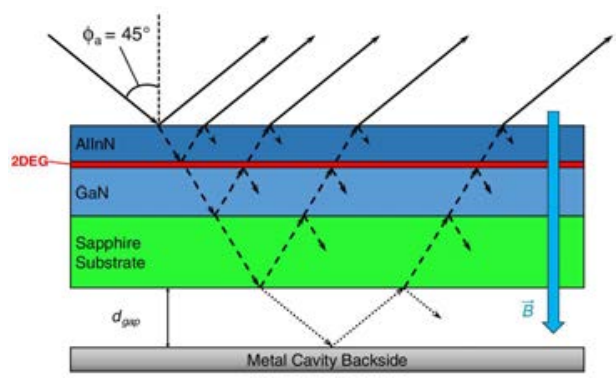

Figure 3.13: Schematic representation of the cavity-enhanced optical Hall effect principle demonstrated for a III-nitride 2DEG heterostructure as an example. Reprinted with permission from Ref. 165.

In the presence of a static magnetic field (B), the motion of the free charge carrier is dominated by the Lorentz force. The reflected electromagnetic plane wave in this case possesses a small fraction of circularly polarized light and causes polarization-state changes $\left(\mathbf{P}_{\mathrm{FCC}}+\mathbf{P}_{\text {Hall }}\right)$, which contain information on the type of charge carriers, their density, mobility, and effective mass properties (see Fig. 3.12) [164].

Mueller matrix formalism is employed to measure the changes in the polarization states induced by the OHE and the OHE data $\delta \mathbf{M}$ are defined as the difference between the Muller matrix elements measured under magnetic field $\pm \mathrm{B}$ $\left(\mathbf{M}_{ \pm B}\right)$ and their corresponding GSE $\left(\mathbf{M}_{0}\right)$

$$
\delta \mathbf{M}=\mathbf{M}_{ \pm \mathbf{B}}-\mathbf{M}_{\mathbf{0}} .
$$

\subsubsection{Cavity-enhanced and in-situ cavity-enhanced THz optical Hall effect}

The cavity-enhanced terahertz optical Hall effect (THz-OHE) is a variety of the OHE technique. The cavity-enhanced OHE uses a tunable air gap between the sample and a metallic permanent magnet. The metal surface of the magnet reflects back the unused radiation emitted out the backside of the sample. As a result, the THz-OHE signal is enhanced (see Fig. 3.13). The physical cause of the enhancement is the radiation reflected back in by the magnet surface undergoing further polarization-state changes when passing the magneto-optic birefringent sample constituent $[165,166]$. THz OHE allows performing an OHE measurements a room temperature and with relatively low-field permanent magnet.

In this thesis, in-situ THz-OHE is further used to measure the effect of ambient conditions on the free charge carrier parameters of epitaxial graphene. For in- 


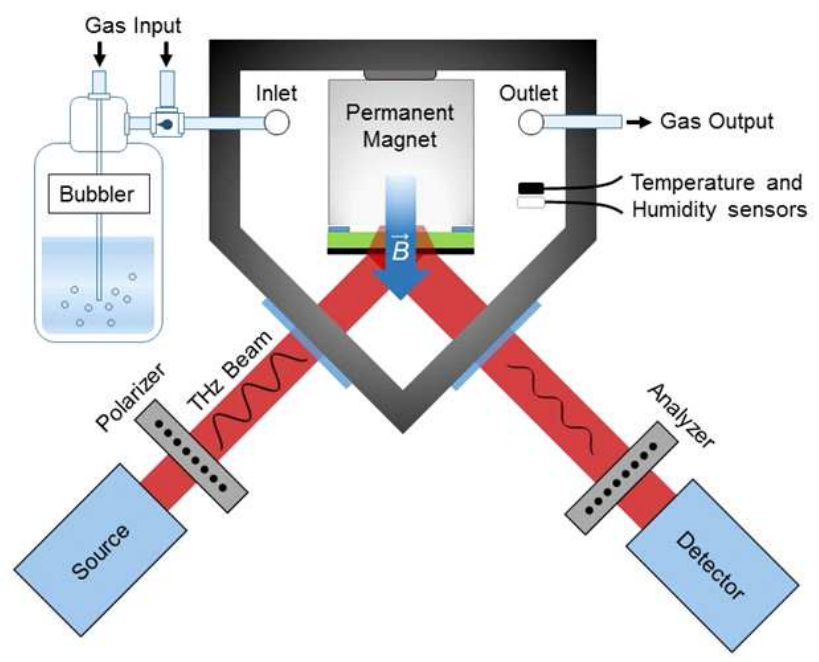

Figure 3.14: Schematic representation of the in-situ cavity-enhanced $\mathrm{THz}$ optical Hall effect. Adapted from paper VI.

situ cavity-enhanced $\mathrm{OHE}$, a gas flow cell is mounted in the $\mathrm{THz}$ ellipsometer. The sample and the magnet are placed inside a gas flow cell (Fig. 3.14). The cell windows are made from homopolymer polypropylene with a thickness of $0.27 \mathrm{~mm}$. The gas follow system is connected to the humidifier enabling precise control of the relative humidity levels. This technique allows the in-situ and the contactless measurements of the free charge carrier properties upon varying the relative humidity or gasses. In this thesis, the in-situ cavity-enhanced OHE has been used to investigate the effect of air and inert gasses on the free charge carrier parameters of epitaxial graphene.

\subsubsection{Optical models}

Spectroscopic ellipsometry is an indirect technique, therefore, a optical model is needed in order to extract the physical parameters of interest. The model is constructed using parametrized model dielectric functions (or tensor) (MDF) of each layer component. A regression analysis is performed via Levenberg-Marquardt algorithm allowing the model parameters to be varied until the model-calculated data are matched as close as possible to the experimental data. The mean squared error used to evaluate the difference between experimental and model generated data is minimized to achieve the best-match model. 


\subsubsection{Dielectric tensor}

The interaction between an electromagnetic wave and a dielectric medium causes the displacement of bound carriers. In linear anisotropic materials, this displacement $(\mathbf{D})$ is a tensor that can be expressed as

$$
\mathbf{D}=\varepsilon_{0} \mathbf{E}+\mathbf{P}=\varepsilon_{0} \mathbf{E}+\chi \mathbf{E}=\varepsilon_{0}(\mathbf{I}+\chi) \mathbf{E}=\varepsilon_{0} \varepsilon \mathbf{E},
$$

$\varepsilon_{0}, \mathbf{E}, \mathbf{P}$, and $\chi$ denote the electric vacuum permittivity, the electric field vector, the electric polarization vector and the electric susceptibility tensor of the medium, respectively. In the Cartesian coordinates $(x, y, z)$, the dielectric tensor can be deduced from (D) and can be expressed as a function of $\chi$ using

$$
\boldsymbol{\varepsilon}=\mathbf{I}+\chi=\mathbf{I}+\left(\begin{array}{lll}
\chi_{x x} & \chi_{x y} & \chi_{x z} \\
\chi_{y x} & \chi_{y y} & \chi_{y z} \\
\chi_{z x} & \chi_{z y} & \chi_{z z}
\end{array}\right)
$$

\subsubsection{Visible to vacuum ultraviolet optical models}

The visible to vacuum ultraviolet (VUV) MDF of epitaxial graphene is described using a sum of Lorentzian and Gaussian oscillators in order to account for the contribution of the critical point (CP) in the DF associated with the Van-Hove singularity at $\sim 4.5 \mathrm{eV}$ [167] such as $\varepsilon=1+\varepsilon_{L}+\varepsilon_{G}$ with

$$
\begin{gathered}
\varepsilon_{L}(E)=\frac{A_{L} \gamma_{L}}{E_{L}^{2}-E^{2}-i \gamma_{L} E}, \\
\Im\left(\varepsilon_{G}(E)\right)=A_{G}\left[e^{-\left(\frac{E-E_{G}}{\sigma}\right)^{2}}+e^{-\left(\frac{E+E_{G}}{\sigma}\right)^{2}}\right], \sigma=\frac{\gamma_{G}}{2 \sqrt{\operatorname{Ln}(2)}},
\end{gathered}
$$

where $A_{L}, E_{L}$, and $\gamma_{L}$ are the amplitude, the center energy and the broadening of the Lorentzian oscillator, respectively and $A_{G}, E_{G}$, and $\gamma_{G}$ are the amplitude, the center energy and the broadening of the Gaussian oscillator, respectively.

The optical model used in this thesis to describe the optical response of $\mathrm{C}$-face graphene on $4 \mathrm{H}-\mathrm{SiC}(000 \overline{1})$ in the visible-VUV spectral range consist of: $4 \mathrm{H}-\mathrm{SiC}$ substrate/interface layer/graphene/roughness layer (Fig. 3.15). The 4H-SiC substrates MDF are described by using a sum of harmonic oscillators [168]. The $\mathrm{SiC}$-graphene interface is described by using a linear effective medium approximation (EMA) [16o] with 50\% graphene and 50\% SiC. The graphene MDF is given above. The surface roughness is modeled by an EMA with $50 \%$ graphene and $50 \%$ air $(\varepsilon=1)$. 


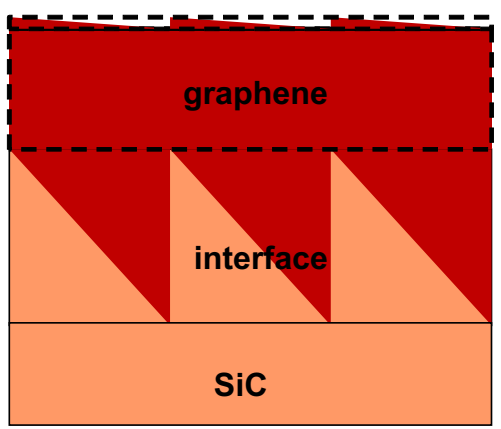

Figure 3.15: A schematic presentation of the optical model used to model the optical response of graphene in the visible-VUV spectral range. Adapted from paper I.

The interface layer accounts for the contributions of: (i) surface roughness and a possible slight off-axis cut of the substrate, (ii) step bunching at the SiC substrate surface, (iii) non-uniform sublimation of silicon from the SiC substrate, and (iv) possible existence of an interface/buffer layer with different structural properties from graphene.

\subsubsection{Mid-infrared/terahertz optical models}

\section{Polar phonons contribution}

In the mid-infrared spectral range (MIR) and at terahertz $(\mathrm{THz})$ frequencies, the $\mathrm{SiC}$ model dielectric tensor has a contribution from its polar lattice vibrations. $4 \mathrm{H}-\mathrm{SiC}$ is uniaxial and its dielectric tensor can be expressed as [169]

$$
\epsilon=\left|\begin{array}{ccc}
\varepsilon_{\|} & 0 & 0 \\
0 & \varepsilon_{\|} & 0 \\
0 & 0 & \varepsilon_{\perp}
\end{array}\right|
$$

where

$$
\epsilon_{j}=\varepsilon_{\infty} \frac{\omega_{L O, j}^{2}-\omega^{2}-i \omega \gamma_{j}}{\omega_{T O, j}^{2}-\omega^{2}-i \omega \gamma_{j}},
$$

with $j=\perp$, $\|$, where $\varepsilon_{\infty}, \omega_{L O}, \gamma_{L O}, \omega_{T O}, \gamma_{T O}$ are the the high-frequency dielectric constant and the frequency and the broadening values of the longitudinal optical (LO) and the transverse optical (TO) phonon modes, respectively. In case of 3C$\operatorname{SiC}(111) \epsilon_{\perp}=\epsilon_{\|}[169]$. 


\section{Free charge carrier contribution}

The dielectric tensor $(\epsilon)$ of a material without a magnetic field can be described

$$
\varepsilon_{\mathrm{B}=0}=\mathbf{I}+\chi_{\mathrm{B}=0}
$$

In the presence of magnetic field, The dielectric tensor can be expressed as [159, 164]

$$
\varepsilon_{\mathrm{OHE}}( \pm \mathbf{B})=\mathbf{I}+\chi_{\mathbf{B}=0}+\chi_{ \pm \mathbf{B}} .
$$

where $\chi_{ \pm \mathbf{B}}$ is the magnetic field induced electric susceptibility which can be expressed as $\chi_{+}$and $\chi_{-}$, for left and right handed circularly polarized light, respectively. In this case, the OHE tensor can be expressed as $[159,163,164]$

$$
\varepsilon^{M O}=\mathbf{I}+\frac{1}{2}\left(\begin{array}{ccc}
\left(\chi_{+}+\chi_{-}\right) & \mp \mathrm{i}\left(\chi_{+}-\chi_{-}\right) & 0 \\
\pm \mathrm{i}\left(\chi_{+}-\chi_{-}\right) & \left(\chi_{+}+\chi_{-}\right) & 0 \\
0 & 0 & 0
\end{array}\right)
$$

The motion of free charge carrier subjected to a magnetic field and electromagnetic radiation $(\mathbf{E}(\mathbf{t})=\mathbf{E} \exp (-\mathrm{i} \omega t))$ can be described by Newtonian equation of motion [159, 163, 164]

$$
\mathbf{m} \frac{d^{2} r}{d t^{2}}+\mathbf{m} \gamma \frac{d r}{d t}+\mathbf{m} \omega_{0}^{2} r=q \mathbf{E}+q(\mathbf{v} \times \mathbf{B}),
$$

where $\mathbf{m}, \mathrm{q}, \boldsymbol{\gamma}=q / m \boldsymbol{\mu}, r, \mathbf{v}=\frac{d r}{d t}, \omega_{0}$ are the effective mass tensor, the electric charge, the plasma broadening parameter, the spacial coordinate of a free charge carrier, the drifting velocity of a free charge carrier and the resonant frequency of a bound carrier. $\boldsymbol{\mu}$ is the mobility tensor. The drifting velocity of the free charge carrier is considered to be harmonic $\mathbf{v}(\mathbf{t})=\mathbf{v} \exp (-\mathrm{i} \omega t)$ and the free charge carriers current density is

$$
\mathbf{j}=N q \mathbf{v}
$$

where $\mathrm{N}$ is the free charge carrier density parameter. Replacing Eqn. 3.25 and $\mathrm{v}(\mathrm{t})$ into the Eqn. 3.24 gives

$$
\mathbf{E}=\frac{1}{N q}\left[\mathrm{i} \frac{\mathbf{m}}{q \omega}\left(\omega_{0}^{2}-\omega^{2} \mathbf{I}-\mathrm{i} \omega \boldsymbol{\gamma}\right) \mathbf{j}+(\mathbf{B} \times \mathbf{j})\right] .
$$

The dielectric tensor and the electric field are related to the conductivity tensor $\sigma$ via $\varepsilon=\mathbf{I}+\frac{\sigma}{i \varepsilon_{0} \omega}$ and $\mathbf{E}=\boldsymbol{\sigma}^{-1} \mathbf{j}$, respectively. The contribution $\chi^{\mathrm{D}}$ to the dielectric tensor of free charge carriers subject to external magnetic field $\mathbf{B}$ can be expressed as $[159,163,164]$

$$
\chi_{i k}^{\mathrm{D}}=\frac{N q^{2}}{\varepsilon_{0}}\left[m_{i k}\left(\omega_{0, i k}^{2}-\omega^{2}-\mathrm{i} \omega \gamma_{i k}\right)-\mathrm{i} \omega \epsilon_{i j k} q B_{j}\right]^{-1},
$$


where $\epsilon_{i j k}$ and $\varepsilon_{0}$ are the Levi-Cevita symbol and the dielectric constant, respectively. The eigenfrequency tensor $\omega_{0}$ of the system for free charge carriers is equal to zero. The contribution of free charge carriers to the dielectric tensor, $\chi_{i k}^{\mathrm{D}}$ (Eqn. 3.27), for isotropic effective mass and conductivity tensors, and magnetic fields aligned along the $z$-axis, can be expressed as [164]

$$
\chi^{\mathrm{D}}=\chi_{\mathrm{B}=0}^{\mathrm{D}}+\chi_{ \pm \mathbf{B}}^{\mathrm{D}}
$$

where $\chi_{\mathrm{B}=0}^{D}$ is the Drude contribution to the dielectric tensor for $B=0$

$$
\chi_{\mathrm{B}=0}^{\mathrm{D}}=-\frac{\omega_{\mathrm{p}}^{2}}{\omega(\omega+\mathrm{i} \gamma)} \mathbf{I}=\chi^{\mathrm{D}} \mathbf{I},
$$

and the isotropic plasma frequency is given by $\omega_{\mathrm{p}}=\sqrt{\frac{N q^{2}}{m \varepsilon_{0}}}$.

The magneto-optic contribution $\chi_{ \pm \mathrm{B}}^{\mathrm{D}}$ to the dielectric tensor for isotropic effective masses and conductivities can be expressed through susceptibility functions for right- and left-handed circularly polarized light $[159,163,164]$

$$
\chi_{ \pm}=\frac{\chi^{\mathrm{D}}}{1 \mp \frac{\omega+\mathrm{i} \gamma}{\omega_{\mathrm{c}}}}
$$

and where the isotropic cyclotron frequency is $\omega_{\mathrm{c}}=\frac{q|B|}{m}$.

\section{Inter-LL-transitions contribution}

In the MIR spectral range and in the presence of a static magnetic field, in addition to the free charge carrier and the polar lattice vibrations, the inter-LL-transition contribution to graphene dielectric tensor has to be considered. The inter-LLtransitions dielectric tensor is expressed using the magneto-optic polarizability functions $\chi_{+}$and $\chi_{-}$for right and left-handed circularly polarized light, respectively [109, 159, 164],

$$
\chi_{ \pm}=\sum_{\mathrm{k}} \frac{A_{ \pm, \mathrm{k}}}{\omega_{ \pm, \mathrm{k}}^{2}-\omega^{2}-\mathrm{i} \omega \gamma_{ \pm, \mathrm{k}}}
$$

where $A_{ \pm, \mathrm{k}}, \omega_{ \pm, \mathrm{k}}$, and $\gamma_{ \pm, \mathrm{k}}$ are the amplitude, energy, and broadening parameters of the $\mathrm{k}_{t h}$ transition, respectively, for (+) right- and (-) left-handed circular polarized light. These parameters depend on the magnetic field. In case of single layer graphene, the polarizabilities for left- and right-handed circularly polarized light are found to be equal $\left(\chi_{+}=\chi_{-}\right)$, and $\varepsilon^{L L}$ is diagonal, while for inter-LLtransitions originating from coupled graphene ( $A B$-stacked graphene layers) a $\chi_{+} \neq \chi_{-}$is determined, and $\varepsilon^{L L}$ is a full-ranked tensor [109]. 


\subsubsection{Instrumentation}

\section{Visible-VUV ellipsometer}

Vacuum ultraviolet (VUV) SE measurements were performed in the photon energy range from 3.5 to $9 \mathrm{eV}$ in nitrogen-purged environment using VUV-302 VASE ellipsometer from J. A. Woollam Co., Inc at the University of Nebraska-Lincoln (U.S.A). The ellipsometry measurements were performed at multiple angles of incidence in order to reduce the correlation between parameters.

\section{Mid-infrared OHE instrument}

The mid-infrared (MIR) OHE measurements were carried out at the University of Nebraska-Lincoln (U.S.A), using the MIR sub-system of the integrated optical Hall effect instrument. The integrated OHE instrument is based on a rotating analyzer ellipsometers design, allowing the measurement of the upper left $3 \times 3$ block of the Mueller matrix $\left(M_{i j}, i, j=1,2,3\right)$. The MIR sub-system employs a Bruker Vertex V70 FTIR-spectrometer, which covers the spectral range $600-8000 \mathrm{~cm}^{-1}$. OHE measurements (with the superconducting magnet) can be carried out at a $45^{\circ}$ angle of incidence of the MIR radiation onto the sample. The magnetic field can be varied between 0 and $8 \mathrm{~T}$ and is aligned parallel to the reflected beam, resulting in a magnetic field $B_{\perp}=B / \sqrt{2}$ parallel to the sample normal. The sample temperature can be varied between 1.5 and $300 \mathrm{~K}$ [159].

\section{THz-OHE measurements}

The THz cavity-enhanced and in-situ cavity-enhanced OHE (100-1040 GHz) measurements were carried out at the $\mathrm{THz}$ materials analysis center at Linköping University, using a newly build OHE instrument which has similar characteristic as the one used in the mid-infrared OHE [166, 170]. In this instrument, the measurement can be carried out at different angle of incidences from $28^{\circ}$ to $90^{\circ}$. The sample is mounted on a neodymium permanent magnet where the magnetic field is perpendicular to the sample. The measurements were performed at room temperature [170]. 



\section{Chapter 4}

\section{Summary of the results}

The results presented in this thesis concern epitaxial graphene grown on the Siand C-terminated surfaces of $4 \mathrm{H}-\mathrm{SiC}\{0001\}$ and the C-terminated surfaces of $3 \mathrm{C}$ $\mathrm{SiC}(111)$ substrates using high-temperature sublimation. We focus on the structural and electronic properties of epitaxial graphene and address several critical questions regarding the effects of substrate polytype and polarity on graphene thickness, stacking order, interaction with the substrate, free charge carrier properties, and interface structure. Our results contribute to the current understanding of the nature and limitations of epitaxial graphene on $\mathrm{SiC}$. We also demonstrate novel $\mathrm{THz}$ ellipsometry methods for the contactless determination of free charge carrier properties of $2 \mathrm{D}$ materials. The major results can be summarized as follows:

\section{C-face few layer graphene}

\section{Interface properties of few layer graphene on C-face $4 \mathrm{H}-\mathrm{SiC}(000 \overline{1})$}

We have reported for the first time the presence of an amorphous $\mathrm{SiO}_{x}$ interface layer for $\mathrm{C}$-face graphene on $4 \mathrm{H}-\mathrm{SiC}(000 \overline{1})$ and propose a mechanism for its formation. We found that this interface layer forms both on $4 \mathrm{H}-\mathrm{SiC}$ substrates with (Paper I) and without (Paper II) surface polishing defects. The thickness of the interface layer was found to increase with aging in ambient conditions. We also have demonstrated that the interface layer causes the formation of non-ideal Schottky contact behavior for electrical transport between monolayer graphene and $\mathrm{SiC}$, which can limit the potential use of thin C-face graphene (1 to 2 monolayers) in electronic applications.

Decoupling and stacking order of C-face graphene on $4 \mathrm{H}-\mathrm{SiC}(000 \overline{1})$ and $3 \mathrm{C}$ SiC(111)

We have shown that few layer C-face graphene grown on $4 \mathrm{H}-\mathrm{SiC}$ (paper II) and 3C$\mathrm{SiC}$ (paper III) consists of decoupled graphene sheets that behave effectively as a single layer graphene with linearly dispersing bands (Dirac cones) at the graphene 
K point. This fact, together with the high mobility parameters of $23500 \mathrm{~cm}^{2} \mathrm{~V}^{-1} \mathrm{~s}^{-1}$ to $33000 \mathrm{~cm}^{2} \mathrm{~V}^{-1} \mathrm{~s}^{-1}$ (at $1.5 \mathrm{~K}$ ), indicate good prospects for implementing few layer C-face graphene ( $>2 \mathrm{MLs}$ ) in future electronic devices. We also found that azimuth rotation between graphene domains may occur laterally not vertically, as generally believed (Papers II and III). In the presence of polishing defects on the substrate surface, $\mathrm{C}$-face graphene may grow either as an $A B$-stacked or a decoupled stack of layers depending on growth temperature (paper I). This can provide possible explanation for the different stacking of C-face graphene, previously reported in the literature. Finally, we have established the thickness dependence of the MDF of graphene in the visible-VUV spectral range (Paper I).

\section{Origin of Landau level splitting in C-face graphene on $4 \mathrm{H}-\mathrm{SiC}(000 \overline{1})$}

We have investigated the possible reasons for Landau level splitting and suggest that the most likely origin is the presence of vacancy defect in C-face graphene (Paper IV).

\section{Si-face epitaxial monolayer to multiple layers graphene}

\section{Contactless access to free charge carriers}

We have demonstrated for the first time a contactless $\mathrm{THz}$ ellipsometry method for reliable determination of free-charge carrier properties of epitaxial graphene with different number of graphene layers (Paper V).

\section{Effect of environmental gases on free-charge carriers in Si-face graphene}

A novel method for in-situ contactless characterization of free-charge carrier properties of epitaxial graphene under the influence of different gases is demonstrated for the first time (paper VI). The method is based on $\mathrm{THz}$ cavity-enhanced optical Hall effect. We have shown that exposure to an inert gas reverses the electron withdrawal caused by air. The doping trend is essentially repeatable when switching between inert gases and air. We found that the mobility variation as a function of sheet density obtained from the different doping cycles shows a linear dependence. This suggests that the dominant scattering mechanism related to ambient doping originates from charged impurities.

\section{Design of an improved crucible and growth of Si-face graphene}

We have designed a crucible with improved temperature homogeneity and used it to grow Si-face graphene on $4 \mathrm{H}-\mathrm{SiC}(0001)$. The effect of buffer layer growth conditions on the thickness homogeneity and free charge carrier properties of Siface graphene is established. We also demonstrate that at the optimized growth conditions, 99\% monolayer graphene with uniform surface morphology, can be grown over area of $15 \times 10 \mathrm{~mm}^{2}$ (Paper VII). 


\section{References}

[1] "The Nobel Prize in Physics 2010", Nobelprize.org. Nobel Media AB 2014. (Web. 14 Oct 2016).

[2] C. Lee, X. Wei, J. W. Kysar, and J. Hone, Science 321, 385 (2008).

[3] P. R. Wallace, Phys. Rev. 71, 622 (1947).

[4] J. Güttinger, F. Molitor, C. Stampfer, S. Schnez, A. Jacobsen, S. Dröscher, T. Ihn, and K. Ensslin, Reports on Progress in Physics 75, 126502 (2012).

[5] A. H. Castro Neto, F. Guinea, N. M. R. Peres, K. S. Novoselov, and A. K. Geim, Rev. Mod. Phys. 81, 109 (2009).

[6] A. K. Geim and K. S. Novoselov, Nature Materials 6, 183 (2007).

[7] E. McCann and M. Koshino, Reports on Progress in Physics 76, 056503 (2013).

[8] S. D. McCann, E.and Abergel and I. V. Fal'ko, The European Physical Journal Special Topics 148, 91 (2007).

[9] R. R. Haering, Canadian Journal of Physics 36, 352 (1958).

[10] E. McCann, D. S. L. Abergel, and V. I. Falko, Solid State Communications 143, 110 (2007).

[11] E. McCann, Phys. Rev. B 74, 161403 (2006).

[12] E. McCann and V. I. Fal'ko, Phys. Rev. Lett. 96, o868o5 (2006).

[13] M. S. Dresselhaus and G. Dresselhaus, Advances in Physics 51, 1 (2002).

[14] E. McCann and V. I. Fal'ko, Phys. Rev. Lett. 96, o86805 (2006).

[15] J. Hass, F. Varchon, J. E. Millán-Otoya, M. Sprinkle, N. Sharma, W. A. de Heer, C. Berger, P. N. First, L. Magaud, and E. H. Conrad, Phys. Rev. Lett. 100, 125504 (2008). 
[16] J. C. Slonczewski and P. R. Weiss, Phys. Rev. 109, 272 (1958).

[17] J. W. McClure, Phys. Rev. 108, 612 (1957).

[18] J. W. McClure, Phys. Rev. 119, 606 (1960).

[19] M. Orlita and M. Potemski, Semiconductor Science and Technology 25, 063001 (2010).

[20] L. Malard, M. Pimenta, G. Dresselhaus, and M. Dresselhaus, Physics Reports 473, 51 (2009).

[21] J.-A. Yan, W. Y. Ruan, and M. Y. Chou, Phys. Rev. B 77, 125401 (2008).

[22] T. Low and P. Avouris, ACS Nano 8, 1086 (2014).

[23] R. R. Nair, P. Blake, A. N. Grigorenko, K. S. Novoselov, T. J. Booth, T. Stauber, N. M. R. Peres, and A. K. Geim, Science 320, 1308 (2008).

[24] K. F. Mak, J. Shan, and T. F. Heinz, Phys. Rev. Lett. 106, 046401 (2011).

[25] L. Yang, J. Deslippe, C.-H. Park, M. L. Cohen, and S. G. Louie, Phys. Rev. Lett. 103, 186802 (2009).

[26] D. S. L. Abergel and V. I. Fal'ko, Phys. Rev. B 75, 155430 (2007).

[27] T. Ando, Y. Zheng, and H. Suzuura, Journal of the Physical Society of Japan 71, 1318 (2002).

[28] A. B. Kuzmenko, E. van Heumen, F. Carbone, and D. van der Marel, Phys. Rev. Lett. 100, 117401 (2008).

[29] K. F. Mak, M. Y. Sfeir, Y. Wu, C. H. Lui, J. A. Misewich, and T. F. Heinz, Phys. Rev. Lett. 101, 196405 (2008).

[30] K. S. Novoselov, a. K. Geim, S. V. Morozov, D. Jiang, M. I. Katsnelson, I. V. Grigorieva, S. V. Dubonos, and a. a. Firsov, Nature 438, 197 (2005).

[31] Y. B. Zhang, Y. W. Tan, H. L. Stormer, and P. Kim, Nature 438, 201 (2005).

[32] K. S. Novoselov, Z. Jiang, Y. Zhang, S. V. Morozov, H. L. Stormer, U. Zeitler, J. C. Maan, G. S. Boebinger, P. Kim, and A. K. Geim, Science 315, 1379 (2007).

[33] S. V. Morozov, K. S. Novoselov, M. I. Katsnelson, F. Schedin, D. C. Elias, J. A. Jaszczak, and A. K. Geim, Phys. Rev. Lett. 100, 016602 (2008). 
[34] K. S. Novoselov, E. McCann, S. V. Morozov, V. I. Falko, M. I. Katsnelson, U. Zeitler, D. Jiang, F. Schedin, and a. K. Geim, Nat. Phys. 180, 177 (2006).

[35] T. Ohta, A. Bostwick, T. Seyller, K. Horn, and E. Rotenberg, Science 313, 951 (2006).

[36] Y. Zhang, T.-T. Tang, C. Girit, Z. Hao, M. C. Martin, A. Zettl, M. F. Crommie, Y. R. Shen, and F. Wang, NATURE 459, 820 (2009).

[37] E. V. Castro, K. S. Novoselov, S. V. Morozov, N. M. R. Peres, J. M. B. L. dos Santos, J. Nilsson, F. Guinea, A. K. Geim, and A. H. C. Neto, Phys. Rev. Lett. 99, 216802 (2007).

[38] K. F. Mak, C. H. Lui, J. Shan, and T. F. Heinz, Phys. Rev. Lett. 102, 256405 (2009).

[39] E. Kasper, M. Oehme, and J. Lupaca-Schomber, High Ge content SiGe alloys: Doping and contact formation (Harame, D and Caymax, M and Koester, S and Miyazaki, S and Rim, K and Tillack, B and Boquet, J and Cressier, J and Masini, G and Reznicek, A and Takagi, S, 2008), vol. 16 of ECS Transactions, pp. 893-904.

[40] M. Koshino and T. Ando, Phys. Rev. B 77, 115313 (2008).

[41] M. Orlita, C. Faugeras, R. Grill, A. Wysmolek, W. Strupinski, C. Berger, W. A. de Heer, G. Martinez, and M. Potemski, Phys. Rev. Lett. 107, 216603 (2011).

[42] F. Bonaccorso, A. Lombardo, T. Hasan, Z. Sun, L. Colombo, and A. C. Ferrari, Materials Today 15, 564 (2012).

[43] S. Bae, H. Kim, Y. Lee, X. Xu, J.-S. Park, Y. Zheng, J. Balakrishnan, T. Lei, H. R. Kim, Y. I. Song, Y.-J. Kim, K. S. Kim, B. Ozyilmaz, J.-H. Ahn, B. H. Hong, and S. Iijima, Nature nanotechnology 5, 574 (2010).

[44] H. Zhou, W. J. Yu, L. Liu, R. Cheng, Y. Chen, X. Huang, Y. Liu, Y. Wang, Y. Huang, and X. Duan, Nature communications 4, 2096 (2013).

[45] Y. Hao, M. S. Bharathi, L. Wang, Y. Liu, H. Chen, S. Nie, X. Wang, H. Chou, C. Tan, B. Fallahazad, H. Ramanarayan, C. W. Magnuson, et al., Science 342, 720 (2013).

[46] Z. Yan, Z. Peng, and J. M. Tour, Accounts of Chemical Research 47, 1327 (2014). 
[47] A. Pirkle, J. Chan, A. Venugopal, D. Hinojos, C. W. Magnuson, S. McDonnell, L. Colombo, E. M. Vogel, R. S. Ruoff, and R. M. Wallace, Applied Physics Letters 99, 122108 (2011).

[48] J. Kang, D. Shin, S. Bae, and B. H. Hong, Nanoscale 4, 5527 (2012).

[49] J. Chen, Y. Guo, L. Jiang, Z. Xu, L. Huang, Y. Xue, D. Geng, B. Wu, W. Hu, G. Yu, and Y. Liu, Advanced Materials 26, 1348 (2014).

[50] W. Yang, G. Chen, Z. Shi, C.-C. Liu, L. Zhang, G. Xie, M. Cheng, D. Wang, R. Yang, D. Shi, K. Watanabe, T. Taniguchi, Y. Yao, Y. Zhang, and G. Zhang, Nature materials 12, 792 (2013).

[51] K. V. Emtsev, A. Bostwick, K. Horn, J. Jobst, G. L. Kellogg, L. Ley, J. L. McChesney, T. Ohta, S. a. Reshanov, J. Röhrl, E. Rotenberg, A. K. Schmid, D. Waldmann, H. B. Weber, and T. Seyller, Nature materials 8, 203 (2009).

[52] C. Virojanadara, M. Syvjrvi, R. Yakimova, L. I. Johansson, A. A. Zakharov, and T. Balasubramanian, Phys. Rev. B 78, 245403 (2008).

[53] W. A. de Heer, C. Berger, M. Ruan, M. Sprinkle, X. Li, Y. Hu, B. Zhang, J. Hankinson, and E. Conrad, Proceedings of the National Academy of Sciences 108, 16900 (2011).

[54] R. Krithivasan, Y. Lu, J. D. Cressler, J.-S. Rieh, M. H. Khater, D. Ahlgren, and G. Freeman, IEEE Electron Device Letters 27, 567 (2006).

[55] A. Tzalenchuk, S. Lara-Avila, A. Kalaboukhov, S. Paolillo, M. Syvajarvi, R. Yakimova, O. Kazakova, M. JanssenT. J. B., V. Fal'ko, and S. Kubatkin, Nat Nano 5, 186 (2010).

[56] G. R. Yazdi, R. Vasiliauskas, T. Iakimov, A. Zakharov, M. Syväjärvi, and R. Yakimova, Carbon 57, 477 (2013).

[57] C. Mathieu, N. Barrett, J. Rault, Y. Y. Mi, B. Zhang, W. A. de Heer, C. Berger, E. H. Conrad, and O. Renault, Phys. Rev. B 83, 235436 (2011).

[58] L. Johansson, C. Xia, J. Hassan, T. Iakimov, A. Zakharov, S. Watcharinyanon, R. Yakimova, E. Janzén, and C. Virojanadara, Crystals 3, 1 (2013).

[59] Z. R. Robinson, G. G. Jernigan, M. Currie, J. K. Hite, K. M. Bussmann, L. O. Nyakiti, N. Y. Garces, A. Nath, M. V. Rao, V. D. Wheeler, R. L. Myers-Ward, J. A. Wollmershauser, B. N. Feigelson, C. R. Eddy, and D. K. Gaskill, Carbon 81, 73 (2015). 
[6o] K. Bolotin, K. Sikes, Z. Jiang, M. Klima, G. Fudenberg, J. Hone, P. Kim, and H. Stormer, Solid State Communications 146, 351 (2008).

[61] N. Petrone, C. R. Dean, I. Meric, A. M. van der Zande, P. Y. Huang, L. Wang, D. Muller, K. L. Shepard, and J. Hone, Nano Letters 12, 2751 (2012).

[62] A. Tzalenchuk, S. Lara-Avila, A. Kalaboukhov, S. Paolillo, M. Syväjärvi, R. Yakimova, O. Kazakova, T. J. B. M. Janssen, V. Fal'ko, and S. Kubatkin, Nature nanotechnology 5, 186 (2010).

[63] F. Speck, J. Jobst, F. Fromm, M. Ostler, D. Waldmann, M. Hundhausen, H. B. Weber, and T. Seyller, Applied Physics Letters 99 (2011).

[64] B. L. VanMil, R. L. Myers-Ward, J. L. Tedesco, C. R. Eddy, Jr., G. G. Jernigan, J. C. Culbertson, P. M. Campbell, J. M. McCrate, S. A. Kitt, and D. K. Gaskill, Materials Science Forum 615-617, 4 (2009).

[65] C. Berger, Z. Song, X. Li, X. Wu, N. Brown, C. Naud, D. Mayou, T. Li, J. Hass, A. N. Marchenkov, E. H. Conrad, P. N. First, and W. A. de Heer, Science 312, 1191 (2006).

[66] T. Yager, A. Lartsev, R. Yakimova, S. Lara-Avila, and S. Kubatkin, Carbon 87, 409 (2015).

[67] T. Shen, J. J. Gu, M. Xu, Y. Q. Wu, M. L. Bolen, M. A. Capano, L. W. Engel, and P. D. Ye, Applied Physics Letters 95 (2009).

[68] X. Wu, Y. Hu, M. Ruan, N. K. Madiomanana, J. Hankinson, M. Sprinkle, C. Berger, and W. A. de Heer, Applied Physics Letters 95 (2009).

[69] L. Banszerus, M. Schmitz, S. Engels, M. Goldsche, K. Watanabe, T. Taniguchi, B. Beschoten, and C. Stampfer, Nano Letters 16, 1387 (2016).

[70] J. Baringhaus, M. Ruan, F. Edler, A. Tejeda, M. Sicot, A. Taleb-Ibrahimi, A.-P. Li, Z. Jiang, E. H. Conrad, C. Berger, C. Tegenkamp, and W. A. de Heer, Nature 506, 349 (2014).

[71] H. Matsunami and T. Kimoto, Mater. Sci. Eng.: R: Reports 20, 125 (1997).

[72] R. Yakimova, C. Virojanadara, D. Gogova, M. Syväjärvi, D. Siche, K. Larsson, and L. I. Johansson, Materials Science Forum 645-648, 565 (2010).

[73] S. Lilov, Computational Materials Science 1, 363 (1993). 
[74] P. Råback, Modeling of the sublimation growth of silicon carbide crystals, (Center for Scientific Computing, 1999).

[75] G. G. Jernigan, B. L. VanMil, J. L. Tedesco, J. G. Tischler, E. R. Glaser, A. Davidson, P. M. Campbell, and D. K. Gaskill, Nano Letters 9, 2605 (2009).

[76] A. Bostwick, T. Ohta, T. Seyller, K. Horn, and E. Rotenberg, Nature Physics 3, 36 (2006).

[77] T. Ohta, A. Bostwick, J. L. McChesney, T. Seyller, K. Horn, and E. Rotenberg, Phys. Rev. Lett. 98, 206802 (2007).

[78] S. Y. Zhou, G.-H. Gweon, a. V. Fedorov, P. N. First, W. a. de Heer, D.-H. Lee, F. Guinea, a. H. Castro Neto, and a. Lanzara, Nature materials 6, 770 (2007).

[79] K. V. Emtsev, F. Speck, T. Seyller, L. Ley, and J. D. Riley, Phys. Rev. B 77, 155303 (2008).

[8o] J. D. Emery, B. Detlefs, H. J. Karmel, L. O. Nyakiti, D. K. Gaskill, M. C. Hersam, J. Zegenhagen, and M. J. Bedzyk, Phys. Rev. Lett. 111, 215501 (2013).

[81] U. Starke and C. Riedl, Journal of Physics: Condensed Matter 21, 134016 (2009).

[82] F. Hiebel, P. Mallet, F. Varchon, L. Magaud, and J.-Y. Veuillen, PHYSICAL REVIEW B 78 (2008).

[83] J. Robinson, X. Weng, K. Trumbull, R. Cavalero, M. Wetherington, E. Frantz, M. LaBella, Z. Hughes, M. Fanton, and D. Snyder, ACS Nano 4, 153 (2010).

[84] S.-i. Nakamura, T. Kimoto, H. Matsunami, S. Tanaka, N. Teraguchi, and A. Suzuki, Applied Physics Letters 76, 3412 (2000).

[85] M. H. Oliveira, T. Schumann, M. Ramsteiner, J. M. J. Lopes, and H. Riechert, Applied Physics Letters 99, 111901 (2011).

[86] W. Norimatsu and M. Kusunoki, Physica E: Low-dimensional Systems and Nanostructures 42, 691 (2010).

[87] V. Borovikov and A. Zangwill, Phys. Rev. B 80, 121406 (2009).

[88] F. Ming and A. Zangwill, Journal of Physics D: Applied Physics 45, 154007 (2012). 
[89] J. L. Tedesco, B. L. VanMil, R. L. Myers-Ward, J. C. Culbertson, G. G. Jernigan, P. M. Campbell, J. M. McCrate, S. A. Kitt, C. R. Eddy, Jr., and D. K. Gaskill, ECS Transactions 19, 137 (2009).

[9o] G. Prakash, M. A. Capano, M. L. Bolen, D. Zemlyanov, and R. G. Reifenberger, Carbon 48, 2383 (2010).

[91] M. Sprinkle, D. Siegel, Y. Hu, J. Hicks, A. Tejeda, A. Taleb-Ibrahimi, P. Le Fèvre, F. Bertran, S. Vizzini, H. Enriquez, S. Chiang, P. Soukiassian, C. Berger, W. A. de Heer, A. Lanzara, and E. H. Conrad, Phys. Rev. Lett. 103, 226803 (2009).

[92] C. Faugeras, A. Nerrire, M. Potemski, A. Mahmood, E. Dujardin, C. Berger, and W. A. de Heer, Applied Physics Letters 92 (2008).

[93] D. A. Siegel, C. G. Hwang, A. V. Fedorov, and A. Lanzara, Phys. Rev. B 81, 241417 (2010).

[94] C. Mathieu, N. Barrett, J. Rault, Y. Y. Mi, B. Zhang, W. A. de Heer, C. Berger, E. H. Conrad, and O. Renault, Phys. Rev. B 83, 235436 (2011).

[95] J. Hass, W. A. de Heer, and E. H. Conrad, Journal of Physics: Condensed Matter 20, 323202 (2008).

[96] J. Hicks, K. Shepperd, F. Wang, and E. H. Conrad, Journal of Physics D: Applied Physics 45, 154002 (2012).

[97] M. L. Sadowski, G. Martinez, M. Potemski, C. Berger, and W. A. de Heer, Phys. Rev. Lett. 97, 266405 (2006).

[98] M. Orlita, C. Faugeras, P. Plochocka, P. Neugebauer, G. Martinez, D. K. Maude, A.-L. Barra, M. Sprinkle, C. Berger, W. A. de Heer, and M. Potemski, Phys. Rev. Lett. 101, 267601 (2008).

[99] D. Sun, C. Divin, C. Berger, W. A. de Heer, P. N. First, and T. B. Norris, Phys. Rev. Lett. 104, 136802 (2010).

[100] W. A. de Heer, C. Berger, X. Wu, P. N. First, E. H. Conrad, X. Li, T. Li, M. Sprinkle, J. Hass, M. L. Sadowski, M. Potemski, and G. Martinez, Solid State Communications 143, 92 (2007).

[101] J. Kedzierski, P. L. Hsu, P. Healey, P. W. Wyatt, C. L. Keast, M. Sprinkle, C. Berger, and W. A. de Heer, IEEE Transactions on Electron Devices 55, 2078 (2008). 
[102] R. Puybaret, J. Hankinson, J. Palmer, C. Bouvier, A. Ougazzaden, P. L. Voss, C. Berger, and W. A. de Heer, Journal of Physics D: Applied Physics 48, 152001 (2015).

[103] X. Wu, Y. Hu, M. Ruan, N. K. Madiomanana, J. Hankinson, M. Sprinkle, C. Berger, and W. A. de Heer, Applied Physics Letters 95, 223108 (2009).

[104] J. L. Tedesco, G. G. Jernigan, J. C. Culbertson, J. K. Hite, Y. Yang, K. M. Daniels, R. L. Myers-Ward, C. R. Eddy, J. A. Robinson, K. A. Trumbull, M. T. Wetherington, P. M. Campbell, and D. K. Gaskill, Applied Physics Letters 96 (2010).

[105] D. K. Gaskill, J. K. Hite, J. C. Culbertson, G. G. Jernigan, J. L. Tedesco, L. O. Nyakiti, V. D. Wheeler, R. L. Myers-Ward, N. Y. Garces, and C. R. Eddy, Jr., Materials Science Forum 679-680, 789 (2011).

[106] J. K. Hite, M. E. Twigg, J. L. Tedesco, A. L. Friedman, R. L. Myers-Ward, C. R. Eddy, and D. K. Gaskill, Nano Letters 11, 1190 (2011).

[107] N. Camara, G. Rius, J.-R. Huntzinger, A. Tiberj, L. Magaud, N. Mestres, P. Godignon, and J. Camassel, Applied Physics Letters 93, 263102 (2008).

[108] M. Orlita, C. Faugeras, J. Borysiuk, J. M. Baranowski, W. Strupiński, M. Sprinkle, C. Berger, W. A. de Heer, D. M. Basko, G. Martinez, and M. Potemski, Phys. Rev. B 83, 125302 (2011).

[109] P. Kühne, V. Darakchieva, R. Yakimova, J. D. Tedesco, R. L. Myers-Ward, C. R. Eddy, D. K. Gaskill, C. M. Herzinger, J. A. Woollam, M. Schubert, and T. Hofmann, Phys. Rev. Lett. 111, 077402 (2013).

[110] Luxmi, N. Srivastava, G. He, R. M. Feenstra, and P. J. Fisher, Phys. Rev. B 82, 235406 (2010).

[111] R. Colby, M. L. Bolen, M. A. Capano, and E. A. Stach, Applied Physics Letters 99 (2011).

[112] X. Weng, J. A. Robinson, K. Trumbull, R. Cavalero, M. A. Fanton, and D. Snyder, Applied Physics Letters 100 (2012).

[113] J. Kuroki, W. Norimatsu, and M. Kusunoki, e-Journal of Surface Science and Nanotechnology 10, 396 (2012).

[114] J. Borysiuk, J. Soltys, R. Bozek, J. Piechota, S. Krukowski, W. Strupinski, J. Baranowski, and R. Stcepniewski, Physical Review B 85, 1 (2012). 
[115] J. Borysiuk, R. Bozek, K. Grodecki, A. Wysmolek, W. Strupinski, R. Stepniewski, and J. M. Baranowski, Journal of Applied Physics 108 (2010).

[116] R. Yakimova, G. R. Yazdi, T. Iakimov, J. Eriksson, and V. Darakchieva, ECS Transactions 53, 9 (2013).

[117] C. Virojanadara, R. Yakimova, J. Osiecki, M. Syvjrvi, R. Uhrberg, L. Johansson, and A. Zakharov, Surface Science 603, L87 (2009).

[118] R. Yakimova, C. Virojanadara, D. Gogova, M. Syvjrvi, D. Siche, K. Larsson, and L. I. Johansson, Materials Science Forum 645-648, 565 (2010).

[119] L. I. Johansson, S. Watcharinyanon, A. A. Zakharov, T. Iakimov, R. Yakimova, and C. Virojanadara, Phys. Rev. B 84, 125405 (2011).

[120] L. I. Johansson, R. Armiento, J. Avila, C. Xia, S. Lorcy, I. A. Abrikosov, M. C. Asensio, and C. Virojanadara, Scientific Reports 4, 4157 (2014).

[121] L. I. Johansson, C. Xia, J. U. Hassan, T. Iakimov, A. A. Zakharov, S. Watcharinyanon, R. Yakimova, E. Janzn, and C. Virojanadara, Crystals 3, 1 (2013).

[122] C. Bouhafs, V. Darakchieva, I. L. Persson, A. Tiberj, P. O. A. Persson, M. Paillet, A.-A. Zahab, P. Landois, S. Juillaguet, S. Schöche, M. Schubert, and R. Yakimova, Journal of Applied Physics 117 (2015).

[123] V. Darakchieva, A. Boosalis, A. A. Zakharov, T. Hofmann, M. Schubert, T. E. Tiwald, T. Iakimov, R. Vasiliauskas, and R. Yakimova, Applied Physics Letters 102 (2013).

[124] A. Ouerghi, M. Marangolo, R. Belkhou, S. El Moussaoui, M. G. Silly, M. Eddrief, L. Largeau, M. Portail, B. Fain, and F. Sirotti, Phys. Rev. B 82, 125445 (2010).

[125] M. Suemitsu and H. Fukidome, Journal of Physics D: Applied Physics 43, 374012 (2010).

[126] C. Coletti, K. V. Emtsev, A. A. Zakharov, T. Ouisse, D. Chaussende, and U. Starke, Applied Physics Letters 99 (2011).

[127] F. Iacopi, G. Walker, L. Wang, L. Malesys, S. Ma, B. V. Cunning, and A. Iacopi, Applied Physics Letters 102, 011908 (2013).

[128] A. Michon, S. Vzian, A. Ouerghi, M. Zielinski, T. Chassagne, and M. Portail, Applied Physics Letters 97 (2010). 
[129] A. C. Ferrari, J. C. Meyer, V. Scardaci, C. Casiraghi, M. Lazzeri, F. Mauri, S. Piscanec, D. Jiang, K. S. Novoselov, S. Roth, and A. K. Geim, Phys. Rev. Lett. 97, 187401 (2006).

[130] A. C. Ferrari and D. M. Basko, Nature Nanotechnology 8, 235 (2013).

[131] A. Das, S. Pisana, B. Chakraborty, S. Piscanec, S. K. Saha, U. V. Waghmare, K. S. Novoselov, H. R. Krishnamurthy, A. K. Geim, A. C. Ferrari, and A. K. Sood, Nature nanotechnology 3, 210 (2008).

[132] C.-F. Chen, C.-H. Park, B. W. Boudouris, J. Horng, B. Geng, C. Girit, A. Zettl, M. F. Crommie, R. A. Segalman, S. G. Louie, and F. Wang, Nature 471, 617 (2011).

[133] A. Das, B. Chakraborty, S. Piscanec, S. Pisana, A. K. Sood, and A. C. Ferrari, Phys. Rev. B 79, 155417 (2009).

[134] Y. Li, Probing the Response of Two-Dimensional Crystals by Optical Spectroscopy (Springer International Publishing, 2016)chap. Intrinsic Doping Dependence of Raman 2D Mode in Graphene: Signatures of Electron-Electron Interaction, pp. 9-18.

[135] P. Venezuela, M. Lazzeri, and F. Mauri, Phys. Rev. B 84, 035433 (2011).

[136] D. M. Basko, S. Piscanec, and A. C. Ferrari, Phys. Rev. B 80, 165413 (2009).

[137] T. M. G. Mohiuddin, A. Lombardo, R. R. Nair, A. Bonetti, G. Savini, R. Jalil, N. Bonini, D. M. Basko, C. Galiotis, N. Marzari, K. S. Novoselov, A. K. Geim, and A. C. Ferrari, Phys. Rev. B 79, 205433 (2009).

[138] S. Manster, L. M. Jawerth, B. A. Leslie, J. I. Weitz, B. Fabry, and D. A. Weitz, Proceedings of the National Academy of Sciences 110, 12197 (2013).

[139] Z. H. Ni, W. Chen, X. F. Fan, J. L. Kuo, T. Yu, A. T. S. Wee, and Z. X. Shen, Phys. Rev. B 77, 115416 (2008).

[140] J. E. Lee, G. Ahn, J. Shim, Y. S. Lee, and S. Ryu, Nat Commun 3, 1024 (2012).

[141] J. A. Robinson, C. P. Puls, N. E. Staley, J. P. Stitt, M. A. Fanton, K. V. Emtsev, T. Seyller, and Y. Liu, Nano Letters 9, 964 (2009).

[142] A. Eckmann, A. Felten, I. Verzhbitskiy, R. Davey, and C. Casiraghi, Phys. Rev. B 88, 035426 (2013). 
[143] I. G. Ivanov, J. U. Hassan, T. Iakimov, A. A. Zakharov, R. Yakimova, and E. Janzn, Carbon 77, 492 (2014).

[144] W. Melitz, J. Shen, A. C. Kummel, and S. Lee, Surface Science Reports 66, I (2011).

[145] E. Meyer, H. J. Hug, and R. Bennewitz, Introduction to Scanning Probe Microscopy (Springer Berlin Heidelberg, 2004), pp. 1-13.

[146] S. Sonde, F. Giannazzo, V. Raineri, R. Yakimova, J.-R. Huntzinger, A. Tiberj, and J. Camassel, Phys. Rev. B 80, 241406 (2009).

[147] F. Giannazzo, V. Raineri, and E. Rimini, Scanning Probe Microscopy in Nanoscience and Nanotechnology 2 (Springer Berlin Heidelberg, 2011)chap. Transport Properties of Graphene with Nanoscale Lateral Resolution, pp. $247^{-285}$.

[148] J. Eriksson, R. Pearce, T. Iakimov, C. Virojanadara, D. Gogova, M. Andersson, M. Syvjrvi, A. Lloyd Spetz, and R. Yakimova, Applied Physics Letters 10o, 241607 (2012).

[149] ELMITEC-LEEM III instrument, http://www.elmitec.de/Leem.php? bereich=Bereich=Highlights (accessed October 04, 2016).

[150] J. dela Figuera and K. F. McCarty, Surface Science Techniques (Springer Berlin Heidelberg, 2013)chap. Low-Energy Electron Microscopy, pp. 531-561.

[151] N. Barrett, J. E. Rault, J. L. Wang, C. Mathieu, A. Locatelli, T. O. Mentes, M. A. Nio, S. Fusil, M. Bibes, A. Barthlmy, D. Sando, W. Ren, et al., Journal of Applied Physics 113, 187217 (2013).

[152] K. L. Man and M. S. Altman, Journal of Physics: Condensed Matter 24, 314209 (2012).

[153] F. Jona, J. A. S. Jr, and W. S. Yang, Reports on Progress in Physics 45, 527 (1982).

[154] M. Xie, Structural and elastic properties of InN and InAlN with different surface orientations and doping, (Linköping University Electronic Press, 2012).

[155] S. Hüfner, Introduction and Basic Principles (Springer Berlin Heidelberg, Berlin, Heidelberg, 2003), pp. 1-6o.

[156] A. Zakharov, A.Mikkelsen, and J. Andersen, Journal of Electron Spectroscopy and Related Phenomena 185, 417 (2012). 
[157] A. Locatelli and E. Bauer, Journal of Physics: Condensed Matter 20, 093002 (2008).

[158] C. Xia, Characterizations of as grown and functionalized epitaxial graphene grown on SiC surfaces (Linköping University Electronic Press, 2015), pp. 20-30.

[159] P. Kühne, C. M. Herzinger, M. Schubert, J. A. Woollam, and T. Hofmann, Review of Scientific Instruments 85 (2014).

[16o] H. Fujiwara, Spectroscopic Ellipsometry, (John Wiley \& Sons, New York, 2007).

[161] R. M. Azzam and N. M. Bashara, Ellipsometry and Polarized Light, (NorthHolland Publ. Co., Amsterdam, 1984).

[162] T. Hofmann, C. M. Herzinger, C. Krahmer, K. Streubel, and M. Schubert, physica status solidi (a) 205, 779 (2008).

[163] M. Schubert, T. Hofmann, and C. M. Herzinger, J. Opt. Soc. Am. A 20, 347 (2003).

[164] M. Schubert, P. Kühne, V. Darakchieva, and T. Hofmann, J. Opt. Soc. Am. A 33, 1553 (2016).

[165] S. Knight, S. Schöche, V. Darakchieva, P. Kühne, J.-F. Carlin, N. Grandjean, C. M. Herzinger, M. Schubert, and T. Hofmann, Opt. Lett. 40, 2688 (2015).

[166] N. Armakavicius, J.-T. Chen, T. Hofmann, S. Knight, P. Khne, D. Nilsson, U. Forsberg, E. Janzn, and V. Darakchieva, physica status solidi (c) 13, 369 (2016).

[167] T. Stauber, N. M. R. Peres, and A. K. Geim, Phys. Rev. B 78, o85432 (2008).

[168] A. Boosalis, T. Hofmann, V. Darakchieva, R. Yakimova, and M. Schubert, Applied Physics Letters 101, 011912 (2012).

[169] T. E. Tiwald, J. A. Woollam, S. Zollner, J. Christiansen, R. B. Gregory, T. Wetteroth, S. R. Wilson, and A. R. Powell, Phys. Rev. B 6o, 11464 (1999).

[170] N. Armakavicius, J.-T. Chen, T. Hofmann, S. Knight, P. Khne, D. Nilsson, U. Forsberg, E. Janzén, and V. Darakchieva, physica status solidi (c) 13, 369 (2016). 


\section{List of Figures}

1.1 Crystallographic structure of graphene . . . . . . . . . . . 2

1.2 Electronic structure of graphene . . . . . . . . . . . . 3

1.3 Crystallographic and electronic structure $A B$-stacked bilayer graphene . 4

1.4 Band structure of monolayer graphene, $A B$-stacked bilayer graphene and twisted bilayer graphene . . . . . . . . . . . . 6

1.5 Crystal and electronic structure of graphite . . . . . . . . . . . . 7

1.6 Phonon dispersions for monolayer graphene . . . . . . . . . . . 8

1.7 Optical properties and optical transmittance of graphene . . . . . . . . . 9

1.8 Exfoliated graphene and magneto-transport measurements . . . . . . . 11

1.9 Landau level energies as a function of magnetic field for monolayer and bilayer graphene . . . . . . . . . . . . . . . . . . 13

1.10 Schematic representation of LL-energies for graphene at a constant magnetic field showing the inter-, intra- and mixture-LL-transitions . . 14

2.1 Schematic summary of techniques used to fabricate large-area graphene 18

2.2 Stacking sequence of bilayer $\mathrm{Si}-\mathrm{C}$ of the $3 \mathrm{C}-, 4 \mathrm{H}$ and $6 \mathrm{H}-\mathrm{SiC}$ polytypes . 19

2.3 Schematic illustration of sublimation growth method and equilibrium partial pressures at different temperature for volatile Si species . . . . . 20

2.4 LEED diffraction patterns showing the Si-face SiC (ooo1) surface reconstruction upon annealing . . . . . . . . . . . . . . 21

2.5 LEED diffraction patterns showing the C-face SiC (0001) surface reconstruction upon annealing . . . . . . . . . . . . . 22

2.6 Comparison of the surface morphology and thickness uniformity of Si-face EG grown in UHV and in argon environment . . . . . . . . . . . 23

2.7 Comparison of Hall effect mobility versus sheet carrier density Si-face EG and C-face graphene grown in UHV and in argon environment . . . 25

2.8 Thickness uniformity, surface morphology, LEED diffraction patterns, electronic band structure and inter-LL-transition of FL -C-face graphene grown by CCS ............................ 26 
2.9 Histogram of the growth mode of C-face graphene as a function of growth temperature and argon growth pressure, graphene nucleation on screw dislocation, and morphology of FL C-face graphene in argon .

2.10 Inter-Landau-level transition energies in for multilayer $\mathrm{C}$-face graphene grown in argon environment . . . . . . . . . . . . . 28

2.11 Comparison of $\mathrm{Si} / \mathrm{C}$ ratio in the vapor phase as a function of annealing temperature of $\mathrm{SiC}$ in C-rich and Si-rich environments. (b) LEEM image of Si-face EG grown by high-temperature sublimation . . . . . . . . . . . 29

2.12 Temperature distributions inside the growth reactor and the crucible . . 30

2.13 LEEM image, LEED diffraction patterns, micro- and nano-ARPES of FL C-face graphene on $\mathrm{SiC}($ ooo $\overline{1})$ by high-temperature sublimation . . . . . 32

2.14 Comparison uniformity and structural properties of Si-face EG and Cface graphene on 3C-SiC(111) . . . . . . . . . . . . 33

2.15 Reflectivity and AFM topography images of Si-face EG by high-temperature under the best conditions ..................... 34

3.1 Raman spectrum of graphene . . . . . . . . . . . . . . 36

3.2 Effect of electron and hole doping on the $\mathrm{G}$ and $2 \mathrm{D}$ peak . . . . . . . . 37

3.3 Raman spectra in the vicinity of $2 \mathrm{D}$ peak as a function of graphene number of layers for exfoliated and FL C-face graphene . . . . . . . . . 39

3.4 Schematic representation of the reflectance setup $\ldots \ldots \ldots$. . . . 40

3.5 Schematic representation of torsion resonance conductive atomic force microscopy and example of maps . . . . . . . . . . . . . . 42

3.6 Schematic representation of scanning Kelvin probe microscopy . . . . . 43

3.7 Schematic representation of the ELMITEC-LEEM III instrument . . . . . 44

3.8 LEEM image of FL C-face graphene on 3C-SiC(111) and electron reflectivity spectra . . . . . . . . . . . . . . . . . 45

3.9 Schematic representation of a LEED system . . . . . . . . . . . . . 46

3.10 Schematic representation of the XPS emission process . . . . . . . . . . . . . . . . . . .

3.11 Schematic representation of spectroscopic ellipsometry . . . . . . . . . 49

3.12 Optical Hall effect schematic representation . . . . . . . . . . . . 50

3.13 Schematic representation of cavity-enhanced optical Hall effect . . . . . $5^{1}$

3.14 Schematic representation of in-situ cavity-enhanced $\mathrm{THz}$ optical Hall

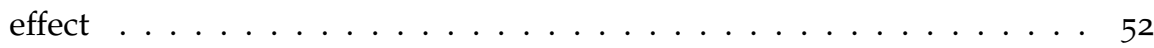

3.15 Schematic representation of the optical model used to model the dielectric function of graphene in the visible VUV spectral range $\ldots . . .54$ 


\section{Papers}

The articles associated with this thesis have been removed for copyright reasons. For more details about these see:

http://urn.kb.se/resolve?urn=urn:nbn:se:liu:diva-132408 